\title{
Run-08 pC polarization analysis October 16, 2008
}

\author{
V. Dharmawardane (New Mexico State Univ.)
}

A. Bazilevsky, G. Bunce, R. Gill, H. Huang, Y. Makdisi, I. Nakagawa, B. Morozov, H. Okada, M. Sivertz, A. Zelenski (Brookhaven National Laboratory)

\author{
I. Alekseev, D. Svirida \\ (Institute of Theoretical and Experimental Physics, Moscow, Russia)
}

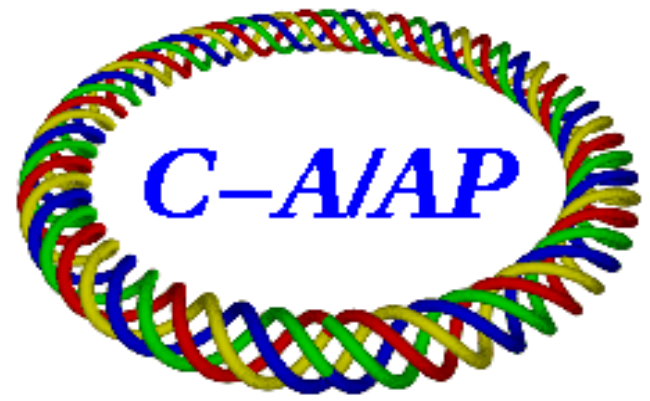

\section{Collider-Accelerator Department Brookhaven National Laboratory Upton, NY 11973}

Notice: This document has been authorized by employees of Brookhaven Science Associates, LLC under Contract No. DE-AC02-98CH10886 with the U.S. Department of Energy. The United States Government retains a nonexclusive, paid-up, irrevocable, world-wide license to publish or reproduce the published form of this document, or allow others to do so, for United States Government purposes. 


\title{
Run-08 pC polarization analysis
}

\section{October 16, 2008}

\author{
V. Dharmawardane \\ New Mexico State University, Las Cruces, NM 88003 \\ A. Bazilevsky, G. Bunce, R. Gill, H. Huang, Y. Makdisi, \\ I. Nakagawa, B. Morozov, H. Okada, M. Sivertz, A. Zelenski \\ Brookhaven National Lab, Upton, NY 11973 \\ I. Alekseev, D. Svirida \\ Institute of Theoretical and Experimental Physics, 117259, Moscow, Russia
}




\section{Introduction}

In this note we will discuss the analysis of RHIC run $08 \mathrm{pC}$ data that were collected during February 14 - March 10, 2008. An analysis method that is similar to Run 05 and Run 06 was adopted for Run 08 analysis (except few minor changes, which are described below). A detailed analysis note and a NIM article that describe the $\mathrm{pC}$ analysis procedure (for run 05 and run 06) can be found elsewhere [1]. In brief, the analysis consists of calibrating the detectors, determining energy corrections ("dead layers"), determining good runs and extracting the polarization from data. Next few sections describe these steps more in detail.

\section{Calibrations}

Each silicon detector used to detect recoil carbon ions are segmented into 12 strips. In total, the pC polarimeter has 6 such detectors mounted at 45, 90, 135 degrees in both left and right sides perpendicular to the beam direction. The silicon Strip Detector (SSD) have a thickness of about $400 \mu \mathrm{m}$, which is sufficient to stop recoil carbon ions of energies up to about $100 \mathrm{MeV}$. Each silicon detector consists of n type bulk material, with a surplus of electron sites in the crystal lattice. The backplane has an aluminum contact over the complete surface. The front face has approximately $150 \mathrm{~nm}$ deep p type silicon strips implanted in the surface. When a charged particle passes through the silicon detector it creates electron-hole pairs in the semiconductor wafer. These electronhole pairs are drifted toward backplane and the surface respectively due to the electric field. The current is measured by small aluminum strips on the surface of each strip.

\subsection{Energy calibration}

Alpha particle sources mounted inside the polarimeter vacuum were used for calibrating the silicon strip detectors. During run 8, four such measurements were taken periodically to account for changes in the detector response. Figs 1 and 2 show calibration constants for both Blue and Yellow detectors. The constants remained relatively stable throughout the run for Blue detectors. However, for the Yellow detector, approximately $15 \%$ change in constants were observed for detector 5 . The exact fill/time at which this occurred was determined by looking at the dead layer values (which will be discussed next). This was possible because any shift in gains will immediately lead to a shift in extracted "dead layers", if wrong calibration constants that do not reflect the proper shift in gain are used. The same could be obtained by fixing "dead layer" values and checking recoil carbon mass. It was determined that the change occurred on February 16 th 2008 that corresponds to Fill 9886. These findings coincide with observation of a jump in detector 5 current around the same time. Therefore, it was decided to use calibration constants extracted using the analysis of data taken on February 13 th until February 16th (Fill 9886) and constants from data taken on February 27 th for runs taken after-wards. 




Figure 1: Gain factor as a function of strip number for blue detectors.

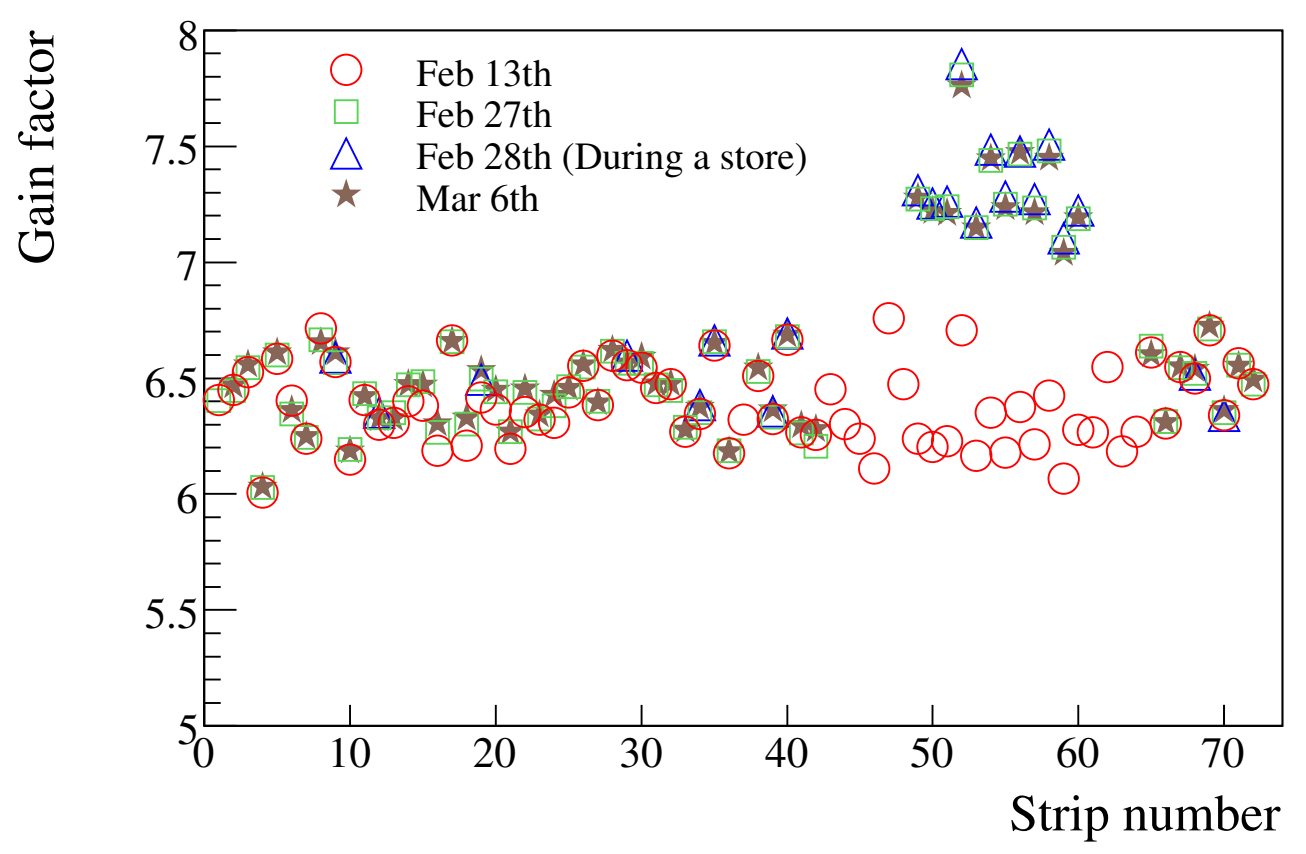

Figure 2: Gain factor as a function of strip number for yellow detectors 


\subsection{Dead layer and t0}

The next step of pC calibration consists of extracting t0, a ToF offset, and dead layer (DL) for each strip in each measurement (run). The $\mathrm{p}+$ doping layer described above is called the "dead layer". The energy lost in this dead layer cannot be measured and is energy dependent and can be described by a known function of energy [1]. Since the $\alpha$ calibration does not effectively probe this surface region of the detectors additional corrections are needed to accurately reconstruct the energy of scattered carbons. These calibration constants were extracted by doing a fit to the "banana" plot, which is the recoil Carbon time-of-flight ( $\mathrm{ToF}$ ) vs energy. The DL parameter carries the meaning of "effective" dead layer and is used to correct the carbon deposited energy to obtain carbon kinetic energy. For run 5 and run 6 the fit was performed for kinetic energy range 400-900 keV. However for run 8 this has to be tightened, since beam-induced pulses caused problems in the polarimetry operation. Fig. 3 shows an example of noise from beam-induced pulses for one run. To remove this background from the data stream

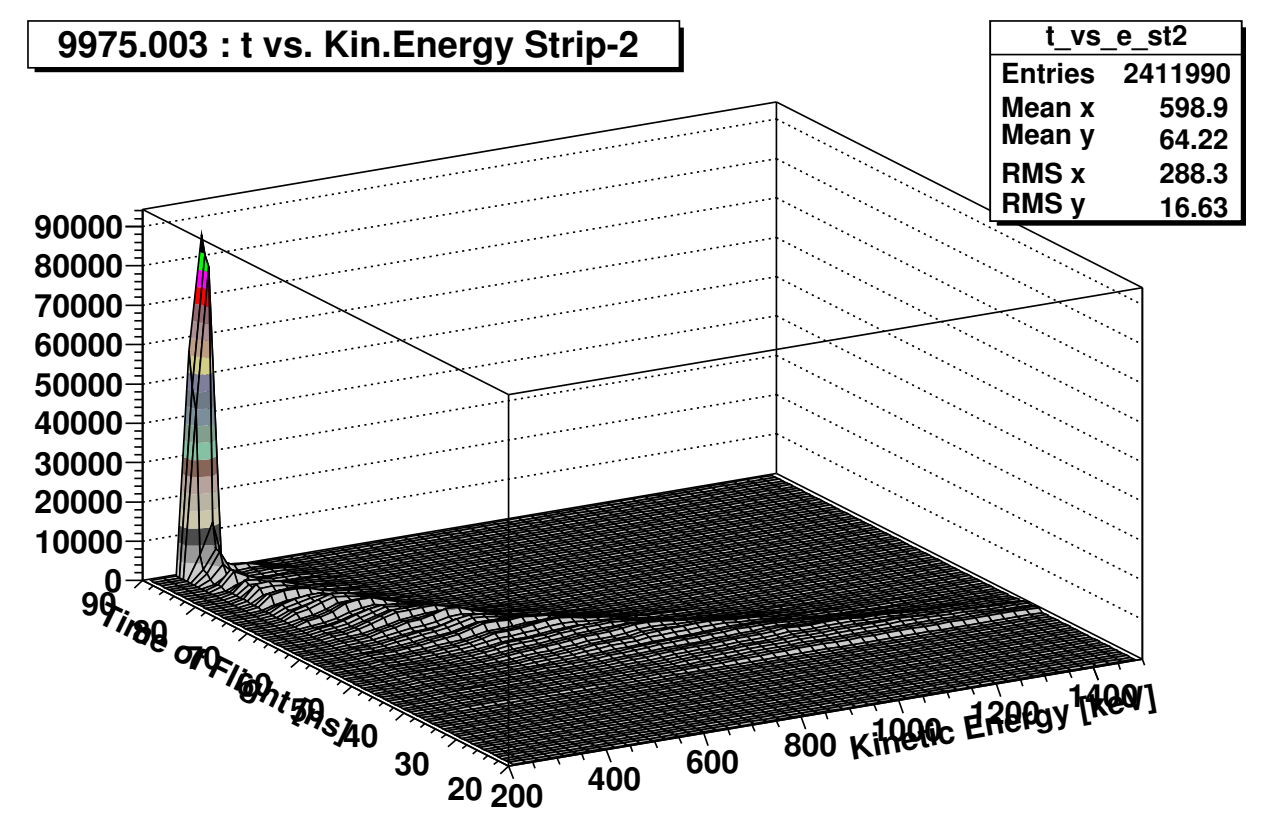

Figure 3: Time of flight vs kinetic energy for a run. Large peak seen at small kinetic energy is due to noise from beam-induced pulses.

time window had to be tightened starting fill 9978, which caused an asymmetric shape in the kinetic energy Vs time distribution. Fig. 4 shows an examples of time of flight Vs kinetic energy distribution before the time window was tightened and after the time window was tightened. Dead layer calibration assumes a symmetric shape in the time Vs kinetic energy distribution. Therefor runs taken starting fill 9978 were calibrated using a kinetic energy cut of 500-900 keV. To ensure that events due to beam induced pulses that may have leaked into the data stream are not included in the analysis, it was also 

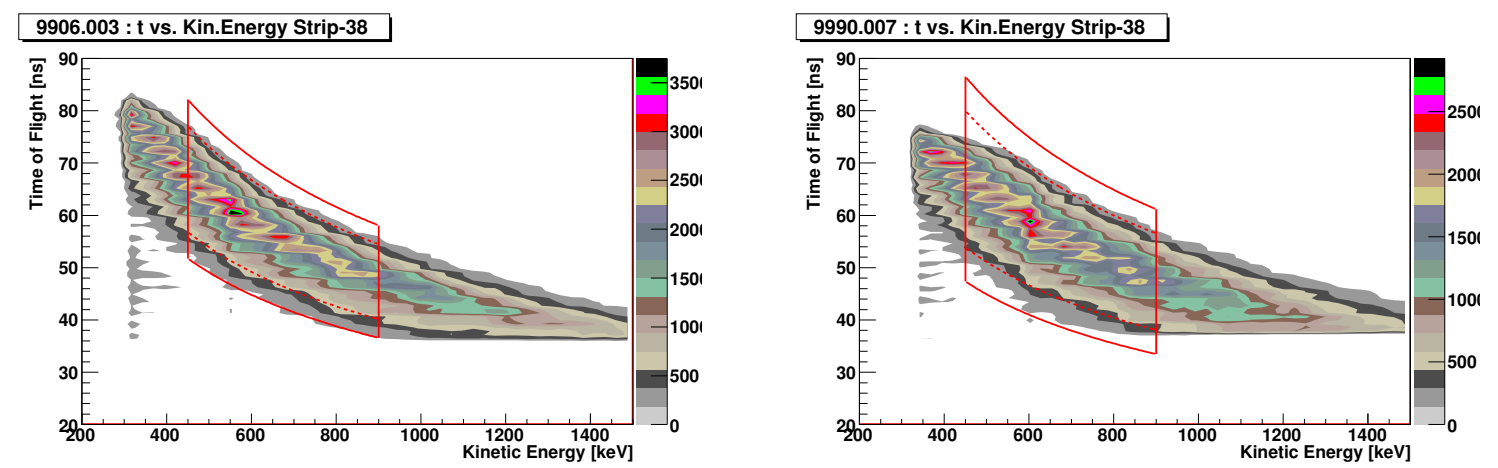

Figure 4: Time of flight Vs kinetic energy distribution before the time window was tightened (left) and after the time window was tightened (right). Asymmetric shape of the banana at low kinetic energy is clearly visible on the right plot.

decided to use a 450-900 keV energy cut instead of the same cut as in run 5 and 6 (400$900 \mathrm{keV}$ ) for runs taken before run 9978. Several studies were performed to determine whether using different cuts for different periods have any significant systematic effect on the relative polarization. It was concluded that using a different cut for dead layer calibration does not cause any significant systematic effect compared to the runs that were calibrated using a 450-900 keV energy cut. A more detailed description of the dead layer and the t0 calibration procedure can be found at [1]. Figs. 5 and 6 show the Run 8 dead layer history for each detector and Fig. 7 shows the average $T 0$ history for both blue and yellow detectors.

\section{Data quality}

The list of QA checks was the same as in Run5 and Run6 pC analysis. It included control of the width and position of the carbon (C) mass peak, as well as $\mathrm{C}$ mass peak position vs its kinetic energy (which detects problems with WFD and/or DAQ and/or in the fit of "banana"; strip by strip variations of the number of events in the "banana"; consistency in bunch-by-bunch asymmetry measurements. All systematic uncertainties from the effects above were estimated to be negligible for the final fill-by-fill polarization measurements, except the energy correction effect (described by DL), which will be described more in detail in the section systematic uncertainties. In general following criteria were used to determine good/bad strips/runs. However, when the deviations are marginal they were considered as good.

- C mass position: Strips with $0.5 \mathrm{GeV}$ deviation from the Carbon mass were not included in the analysis.

- Number of events in banana: Strips outside $20 \%$ from the average were removed. 



Figure 5: Dead layer history for blue detectors.

- C mass width: strips with a deviation above 1.92 sigmas were not included in the analysis.

- Specific luminosity per bunch: Specific luminosity is defined by the ratio between number of events per bunch within kinematic cuts and the intensity per bunch measured by the wall current monitor. The number of events are summed for all active strips for a given bunch. Under perfect conditions specific luminosity per bunch should be a constant for all bunches. However, runs taken with the blue vertical target 5 (Fill numbers : 9942, 9947, 9948 and 9949) showed large deviations from the average for first few bunches. An example of one such run is given in Fig. 9. These runs were taken with a thick target in fills with high beam intensity, leading to extremely high event rates ( $5 \mathrm{MHz}$ for events within banana). The problem might be connected with base line shift in WFDs. However, we didn't find a way to correct this effect, and it was decided not to include any of those runs in the analysis and to borrow results from the HJet analysis for those fills. Unfortunately, we do not have any HJet measurement for fill 9947, therefore we cannot provide any polarization measurement for this fill. For Fill 9949 few runs were taken with a different target, therefore $\mathrm{pC}$ polarization results for this fill can be provided. 

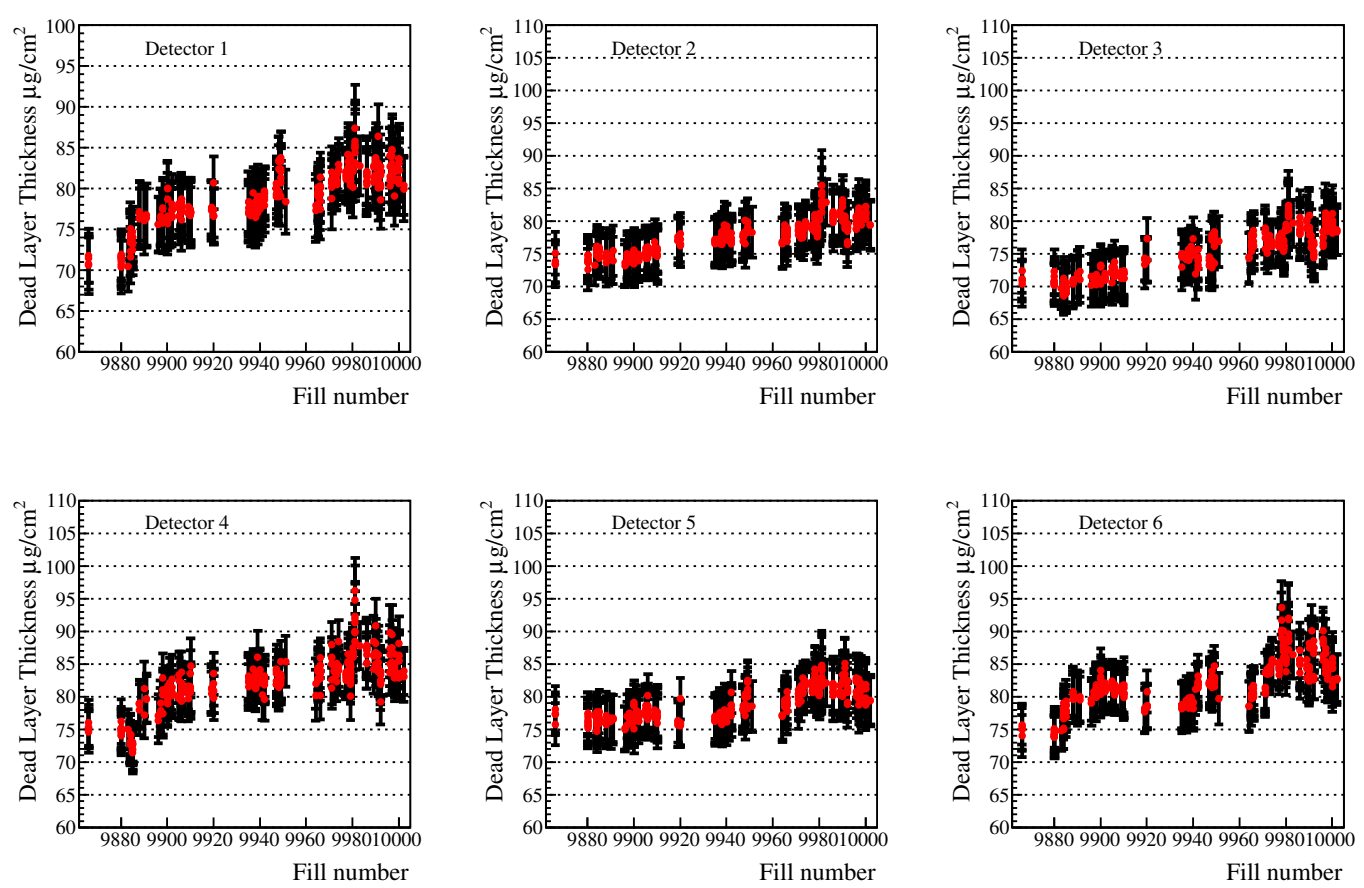

Figure 6: Dead layer history for yellow detectors.
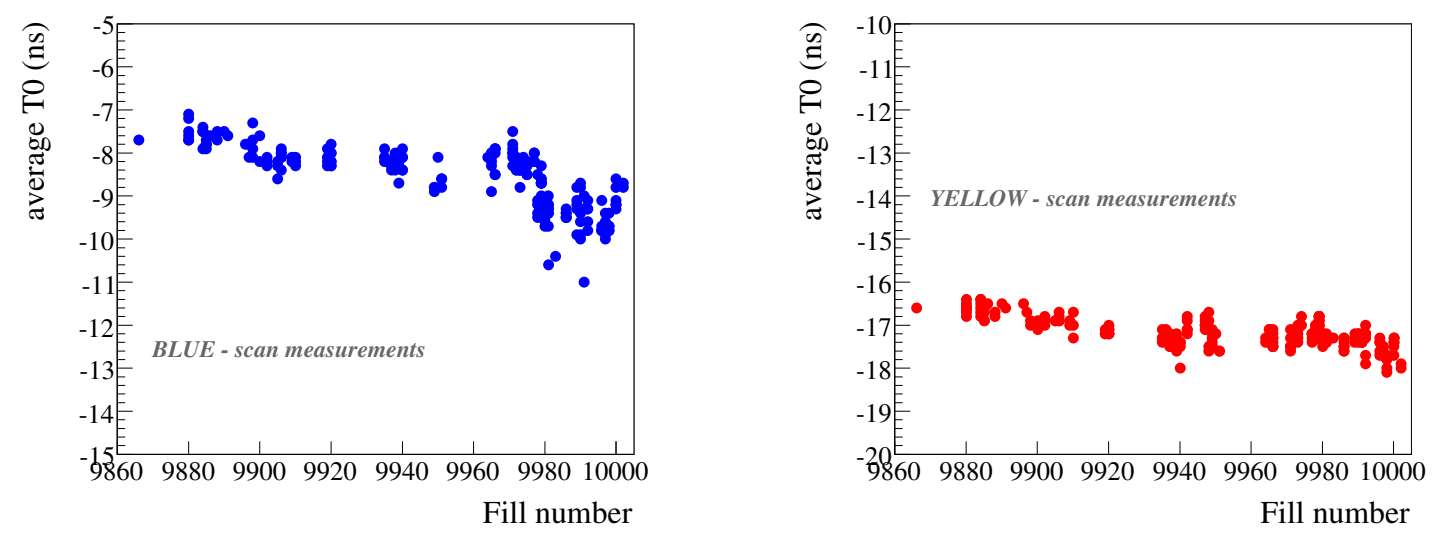

Figure 7: T0 history for blue and yellow detectors.

On total two blue strips and five yellow strips were permanently disabled. Four yellow strips showed a secondary peak near the carbon peak due to electronic jitter and were permanently disabled. One yellow strip was electronically disconnected and was also permanently disabled. The two blue strips that were permanently disabled fell outside the criteria defined for "good quality" throughout the run. In addition many strips were disabled temporarily (in some case just one run and sometimes for several runs in a 
row) for some runs and they were enabled once the strips turned back to normal and fell within the criteria defined above for "good" strips. On maximum, 8 strips (per run) were disabled for some runs. A list of runs that were not included in the analysis due to problems (such as hardware failure and mechanical problems that prevented from making a proper target scan measurement) that cannot be recovered by disabling strips is given elsewhere [2]. However, most of the runs that were not included are simply "Junk" runs or runs with very few or zero statistics and therefore could not be analyzed. Four blue and eleven yellow scan runs that are good otherwise were removed from the analysis due to bad scans (an example of a bad scan is given in Fig. 22. Details regarding scan data will be discussed more in detail later in the text)


Figure 8: An example of quality checks for a typical run that was used in the analysis.

\section{Polarization profile}

For run 08 , pC polarimeter data were taken with the scan mode and fixed target mode. In the scan mode, measurements were performed with vertical targets and horizontal targets, stepping, in x (transverse horizontal coordinate) or y (vertical coordinate) across the beam, with equal measurement time at each step. In latter part of Run8 we used continuous scan mode in the measurements, instead of step wise. It allowed to measure horizontal/vertical polarization profile in each run separately (with limited statistical precision). All the Yellow data were taken with the Vertical targets, which provided horizontal polarization profile measurements. Blue data were taken with both Horizontal 




Figure 9: An example of Carbon yield/ wall current monitor as a function of bunch number for a run that was not included in the analysis.

and Vertical targets. Typically, fixed target measurements were made at the beginning and at the end of each fill with the target positioned at the peak intensity in $\mathrm{x}$ or $\mathrm{y}$, and scan measurements were made approximately once per every two hours at flattop energy. In the scan mode data taken at each target position is analyzed exactly the same way the data are analyzed for the fixed target mode. In general, relative polarization $\left(P_{0}\right)$ and the phase factor phi $\left(\phi_{0}\right)$ are determined by doing a fit that is of the form $P_{0}\left(\sin \left(\phi+\phi_{0}\right)\right)$ to measured asymmetries (Fig 10). For run 8 a similar procedure as run 6 was followed in the analysis of scan data except how the phase factor $\phi_{0}$ is determined for each scan target position. In the analysis of run 6 data $\phi_{0}$ was not kept fixed for all target positions. For run 8 analysis $\phi_{0}$ at each target position was kept fixed at the $\phi_{0}$ obtained by doing a fit to all scan data of that run. Fig 11 compares the effect on the relative polarization extracted using the two methods. As expected this new procedure didn't change the extracted polarization much for target positions with plenty of statistics. However for positions with fewer statistics, a few percent change in the extracted polarization was observed, and in general showed a trend towards a slightly lower polarization, leading to a sharper polarization profile. This effect was confirmed in a simple MC: keeping $\phi_{0}$ parameter free for all scan positions (Run6 approach) leads to a bias in polarization measurements (to higher values) when statistics are low. Fig. 12 shows the average $\phi_{0}$ for each fill for all Run 8 scan measurements.

Similar to run 5 and run 6 , the strategy is to obtain the normalization for pC measurements using absolute polarization measurements with HJet in the fills for which HJet measurement is available, and after that use the properly normalized pC measurements to define the polarization in each physics fill.

To obtain average polarization over the beam intensity distribution in the transverse plane, the knowledge on the polarization profile (polarization vs $\mathrm{x}$ and $\mathrm{y}$ in transverse plane) is necessary. The correction due to polarization profile depends on the ratio of 


\section{Run9935.001: Strip Asymmetry sin(phi) fit}



Figure 10: An example of a fit to asymmetries. Polarization and the "phase factor", $\phi_{0}$, are extracted by doing a fit of the form $P_{0}\left(\sin \left(\phi+\phi_{0}\right)\right)$.

width of the beam intensity profile and beam polarization profile. It can be obtained from the direct measurements of the widths of the profiles, which requires good target positioning during the scan. Another way, which excludes the necessity of the precise target positioning, is a fit of polarization vs event rate (which is proportional to beam intensity) in a scan, by a function $\frac{P}{P_{\max }}=\left(\frac{I}{I_{\max }}\right)^{R}$; here it is assumed that both intensity and polarization profiles have Gaussian shapes with widths $\sigma_{I}$ and $\sigma_{P}$, correspondingly, and at least one point in the scan corresponds to beam maximum intensity; $P_{\max }$ and $I_{\max }$ are polarization and event rate at beam maximum intensity; $R$ is $\left(\frac{\sigma_{I}}{\sigma_{P}}\right)^{2}$. Since many of the $\mathrm{pC}$ measurements showed non-Gaussian intensity profiles, which may be due to target positioning problems, this latter approach was used to extract $P_{\max }$ and $R$ parameters for each fill (Fig 13), which were used to calculate the average beam polarization (when doing normalization to HJet measurements) and for colliding beams: $<P>=\frac{P_{\max }}{\sqrt{(1+R)}}$ and $\langle P\rangle=\frac{P_{\max }}{\sqrt{(1+R / 2)}}$, correspondingly, for one dimensional case. Figs.14 - 18 show $R$ and $P_{\max }$ for all blue and yellow scan data for run 8 as a function of the fill number.

As a consistency check average polarization calculated using $P_{\max }$ and $R$ were compared to the average polarization extracted by simply combining the data taken at each scan position. The average polarization for each fill in the latter case was determined by taking the weighted average of all average polarizations obtained for each run. This will ensure that the assumptions that were made in extracting the fit parameters are correct. 


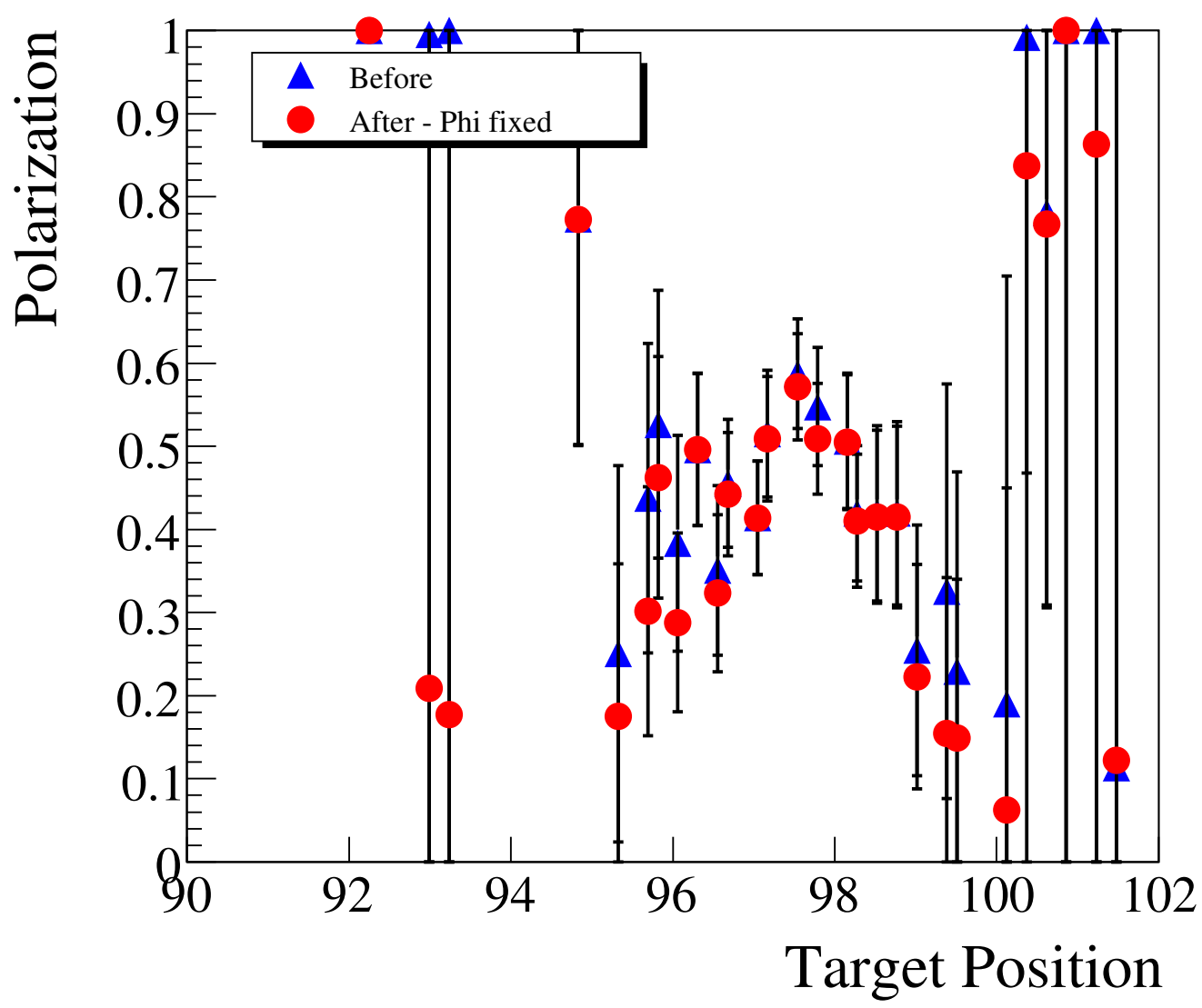

Figure 11: Comparison of polarization extracted by keeping $\phi_{0}$ fixed and letting it vary. More details can be found in the text.

Fig. 19 compares the average polarization calculated using the two methods and the difference in the polarization for the two methods. The difference is approximately 1.4 $\pm 0.3 \%$ and $-0.7 \pm 0.6 \%$ for blue and yellow beams respectively. Slight difference in blue is explained by flatter (non-Gaussian) intensity profiles seen in each scan measurements, which means that effectively during the measurements target didn't move uniformly, but on the average stayed longer near beam center, which led to higher average polarization over a run (so led to a bias in polarization measurements).

\section{Polarization decay}

When combining runs we assume that the polarization decay within a fill is negligible. To make sure that this assumption is true polarization decay for each fill was studied. First, no polarization decay was assumed and a linear fit to polarization was performed to obtain the average polarization. In the case of any polarization decay, an exponential decay 




Fill number

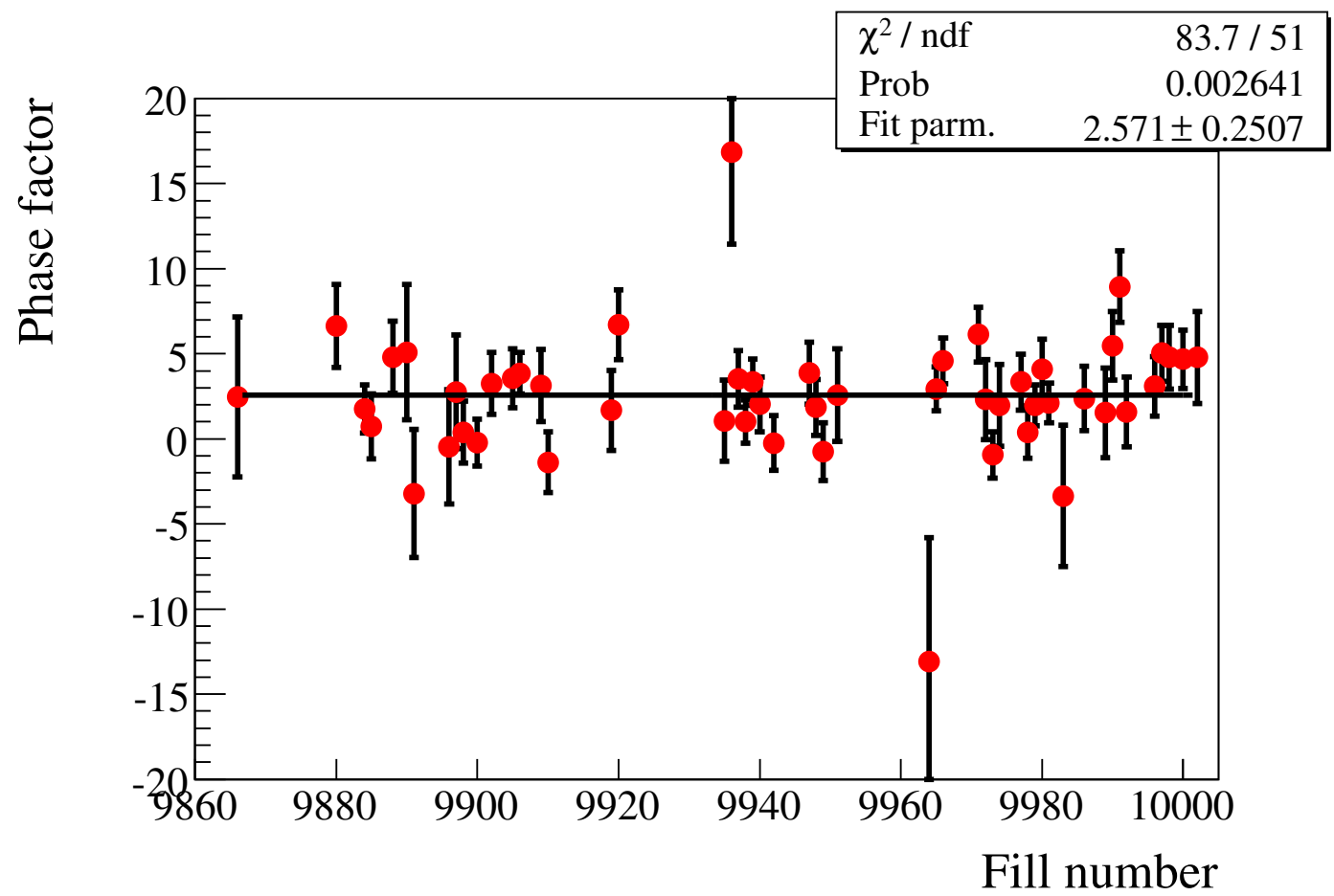

Figure 12: Average $\phi_{0}$ of each fill for all scanned measurements (blue -top plot and yellow - bottom plot). 


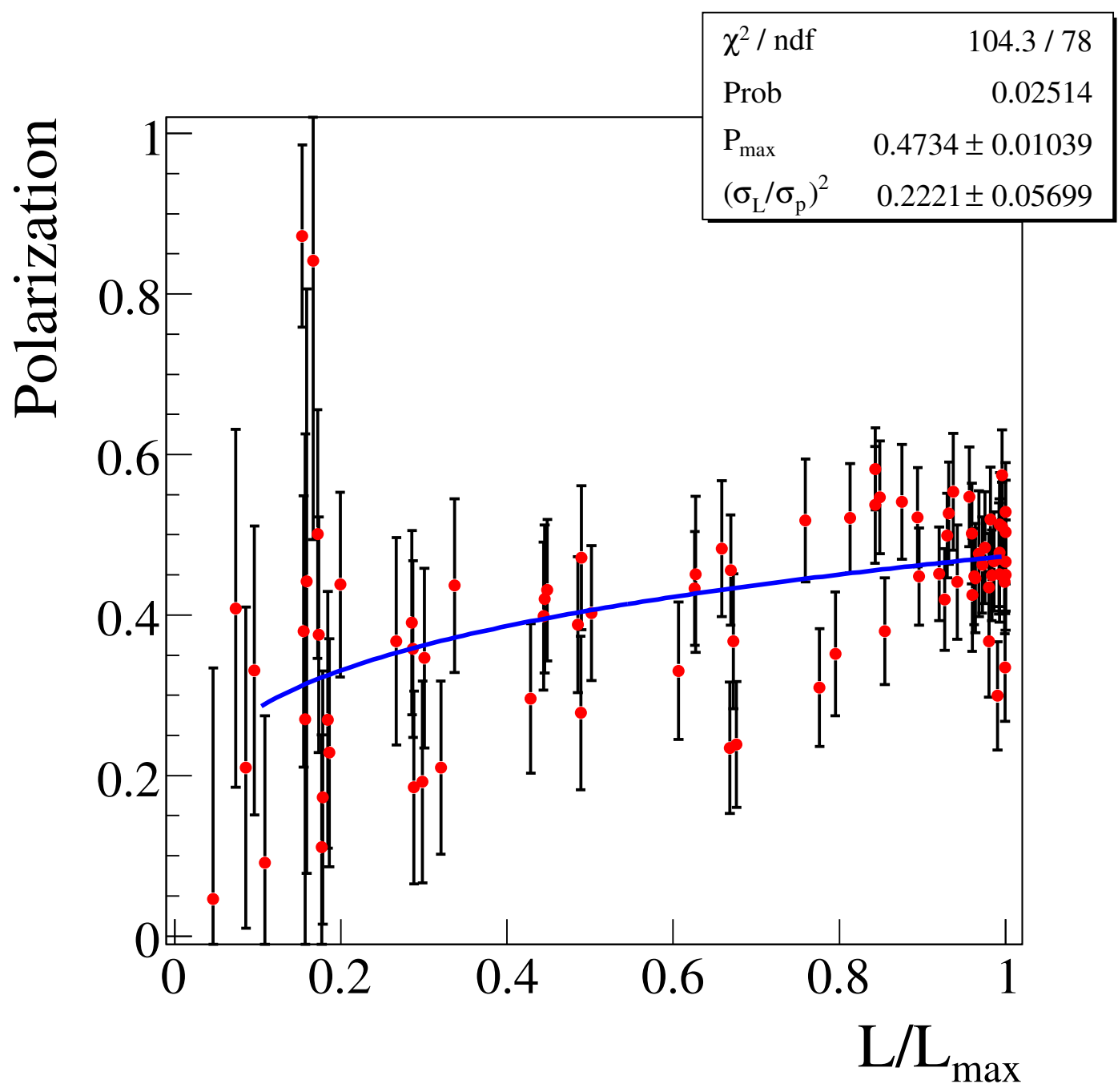

Figure 13: To extract $P_{\max }$ and $R$ a fit to $P$ Vs $\frac{I}{I_{\max }}$ was performed. The plot shows a typical fit to one fill. 


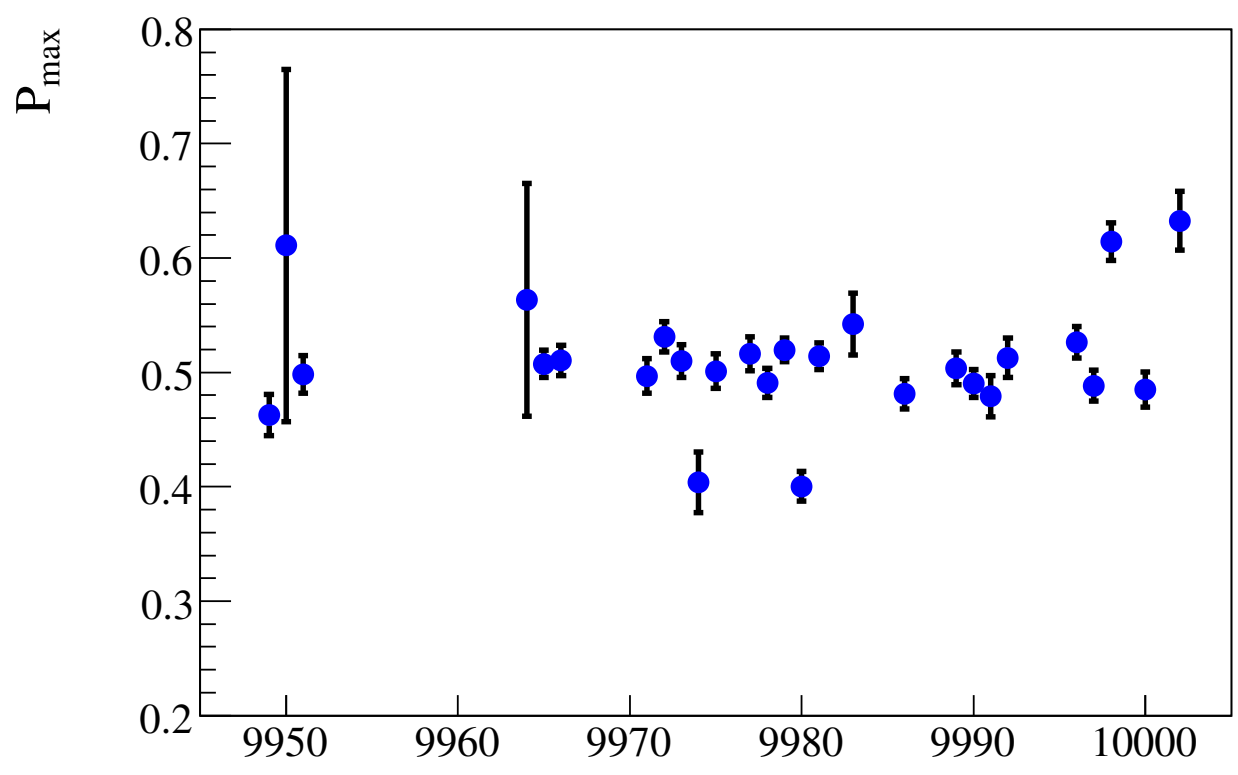

Fill number

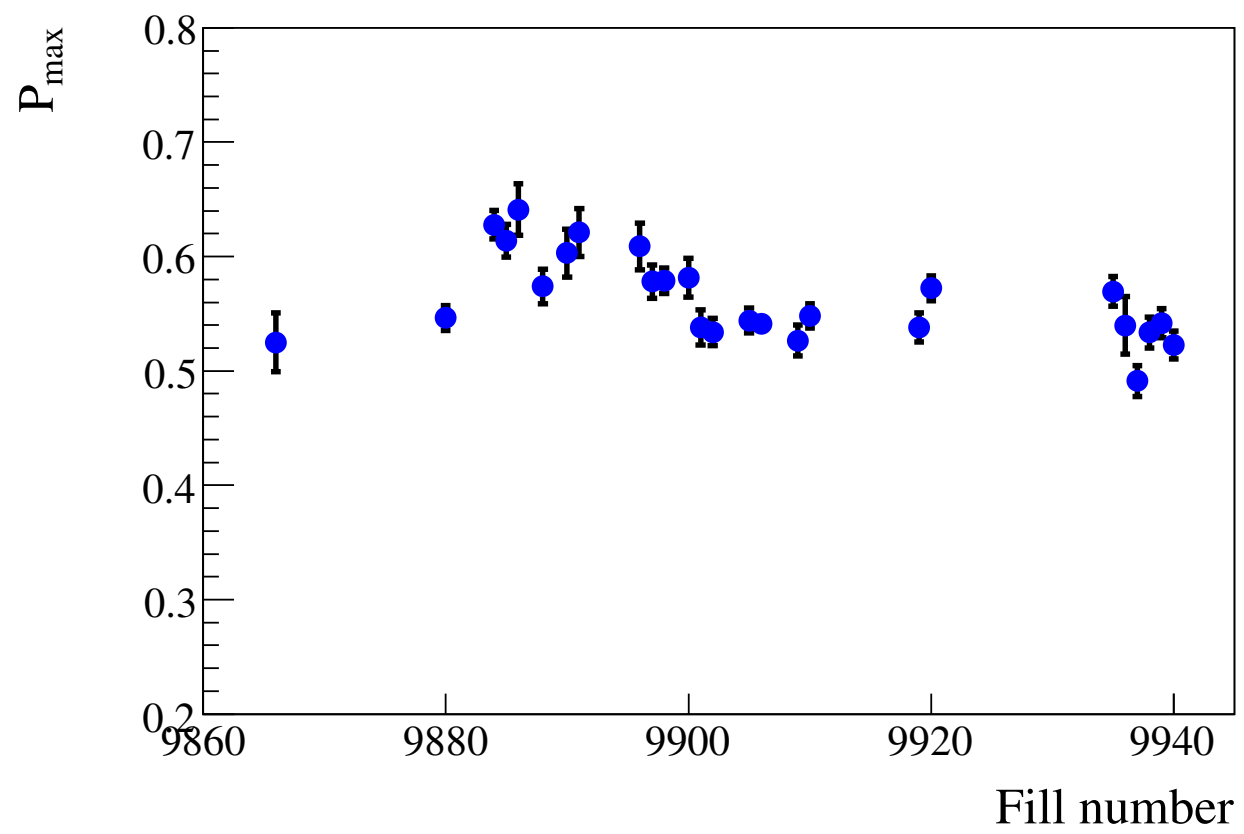

Figure 14: $P_{\max }$ history of (vertical scan (top) and horizontal scan(bottom)) all blue data. 


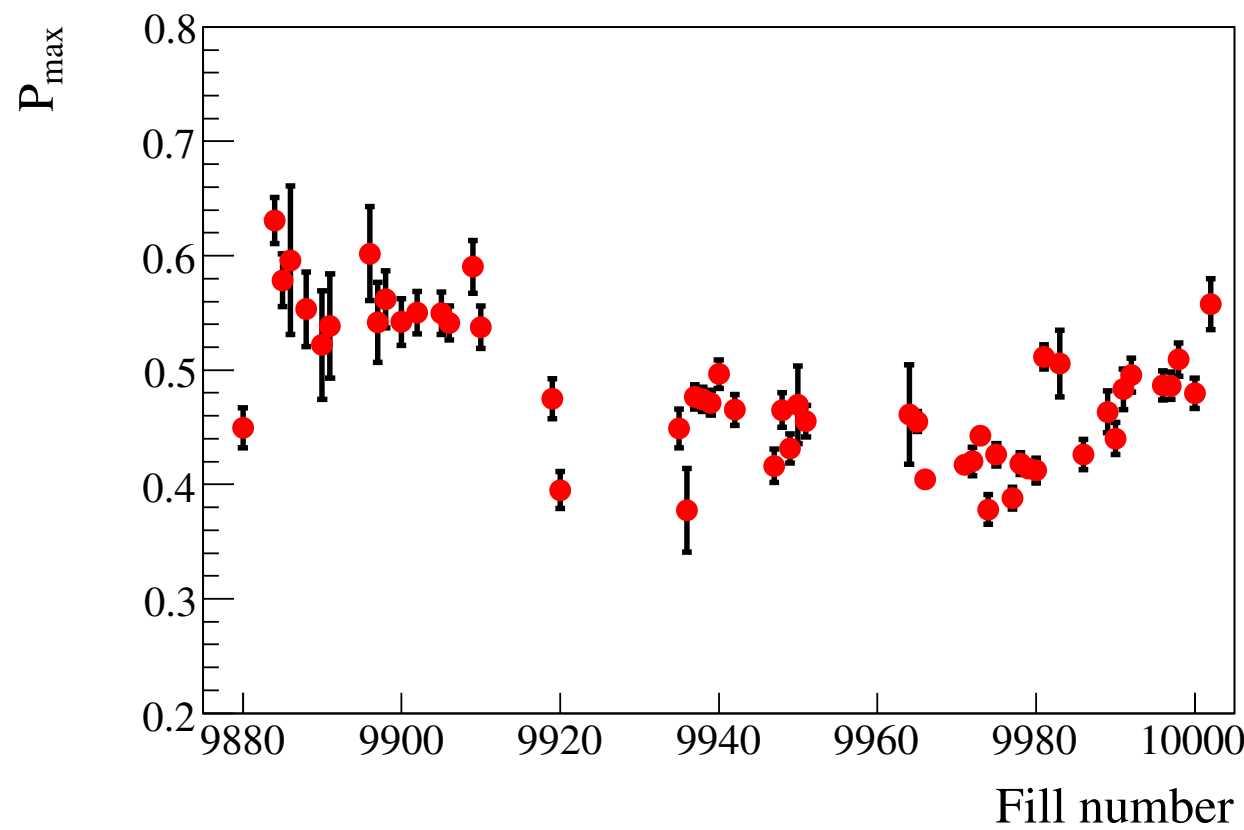

Figure 15: $P_{\max }$ history of (horizontal scan) all yellow data.

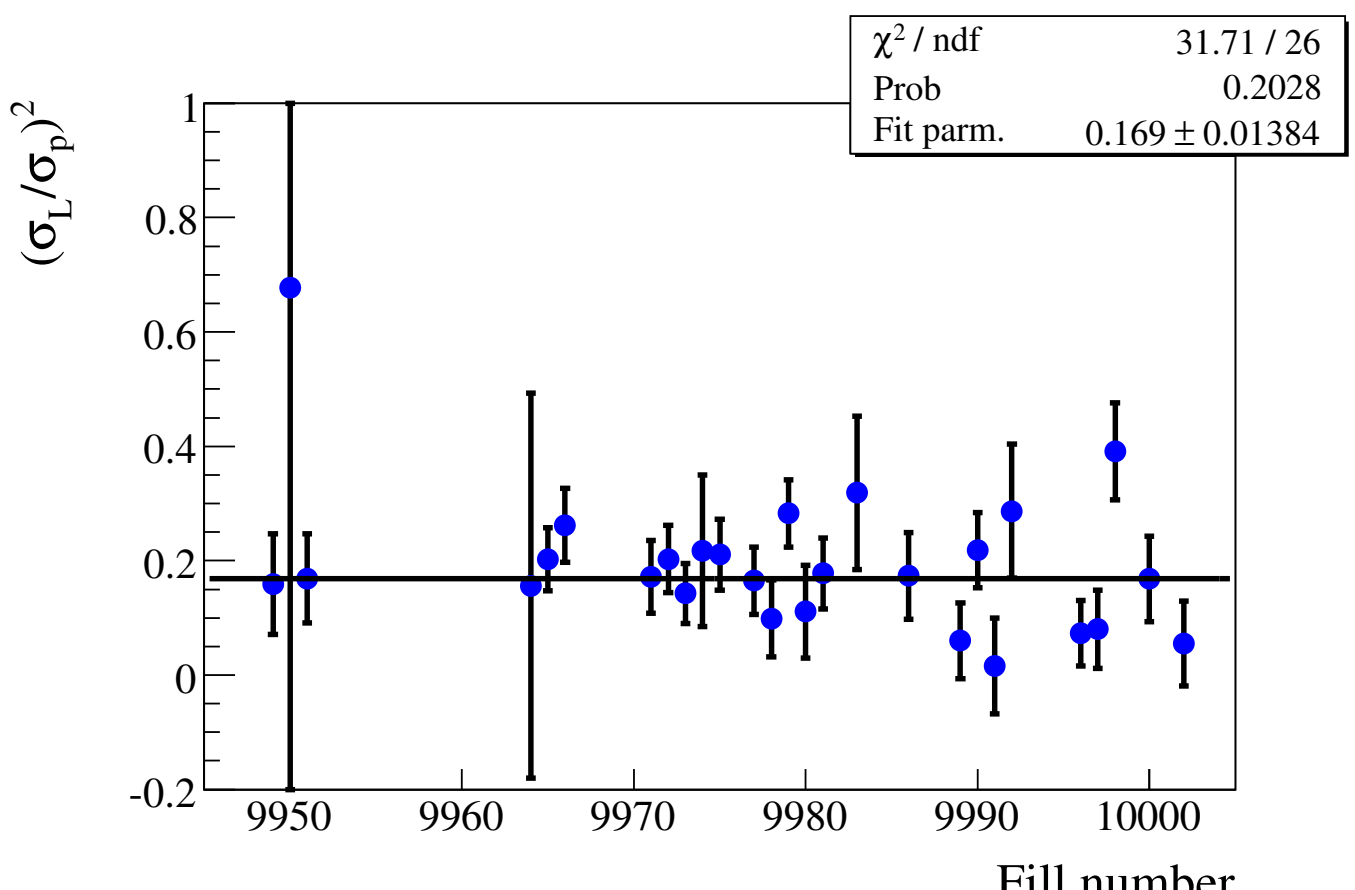

Figure 16: $R$ history of vertical scan blue data. 


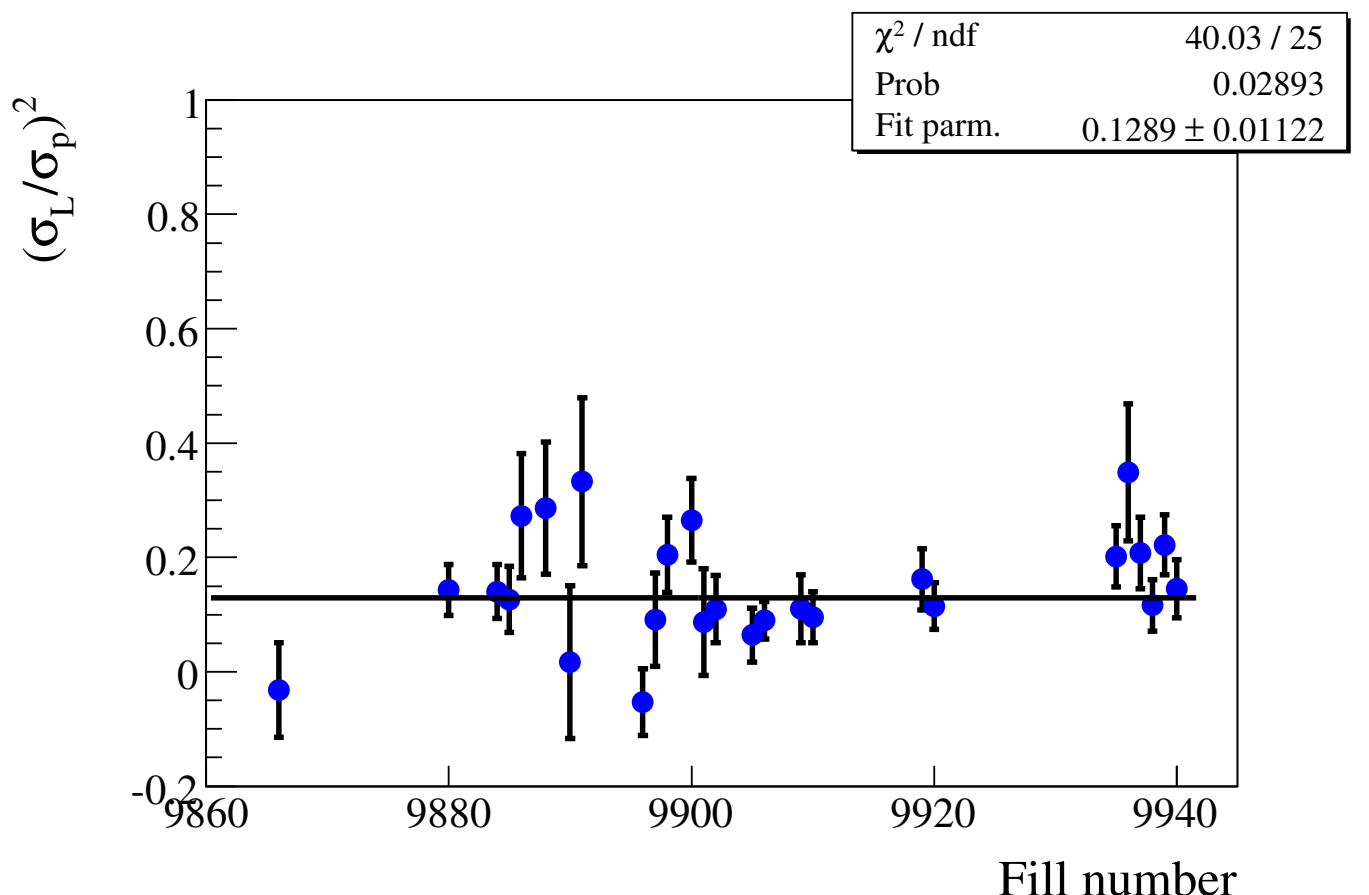

Figure 17: $R$ history of horizontal scan blue data.

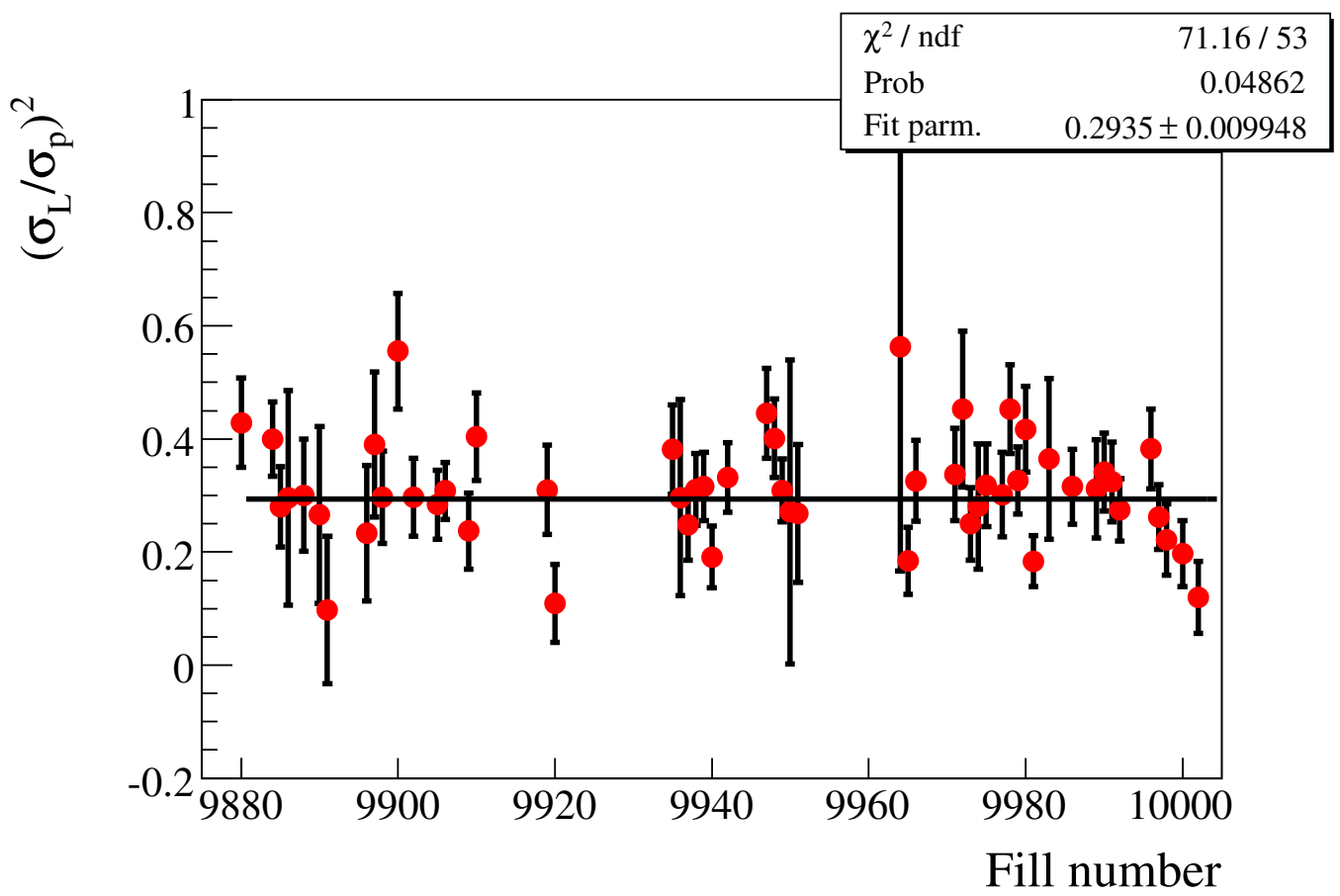

Figure 18: $R$ history of all (horizontal scan) yellow data. 

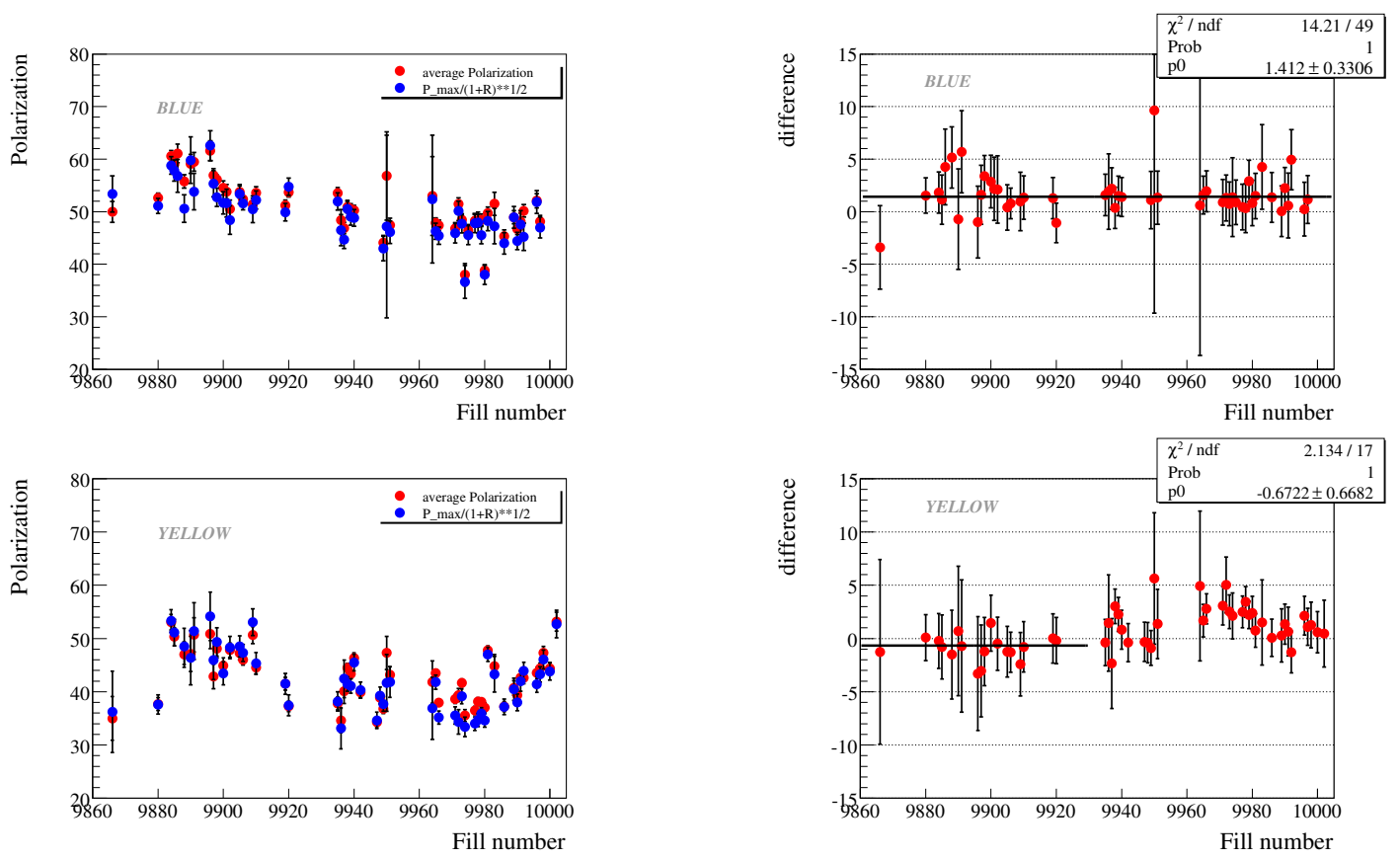

Figure 19: Average polarization calculated using fit parameters $P_{\max }$ and $R ;\langle P\rangle=$ $\frac{P_{\max }}{\sqrt{(1+R)}}$, and combining all scan measurements and the difference between the two methods for blue and yellow beams.

of the polarization was assumed. A fit of the form $P=P_{0} \exp ^{\alpha * t i m e}$, where $\alpha=1 / T_{\text {decay }}$ was performed to obtain the decay time $T_{\text {decay }}$ for each fill. The average polarization for fills that assume an exponential decay is given by: $\langle P\rangle=\frac{P_{0} \int e^{-\alpha P^{* t}} e^{-\alpha_{L} * t} d t}{e^{-\alpha_{L} * t} d t}$, where $\alpha_{L}$ and $\alpha_{P}$ are the inverse of the polarization and luminosity decay (a decay constant of 15 hours for luminosity decay was assumed to obtain the average polarization). Fig. 20 shows an example of a fill that shows large polarization decay and very little polarization decay. Fig. 21 shows the polarization decay for each fill. On average Yellow beam showed a much faster polarization decay than the blue beam. In addition, the average polarization obtained assuming no polarization decay and an exponential decay were compared. As it is evident from Fig. 21 the difference between the two are consistent with zero. It was concluded that any correction due to polarization decay is not necessary when averaging results over a given fill.

\section{Normalization to H-Jet}

To relate HJet measurements to $\mathrm{pC}$ measurements, only $R$ parameter in one direction (vertical or horizontal) is necessary, because the carbon target automatically averages polarization in the other direction. To determine the average pC polarization for each period, the pC polarization for each fill was weighted by number of HJet counts. Runs 

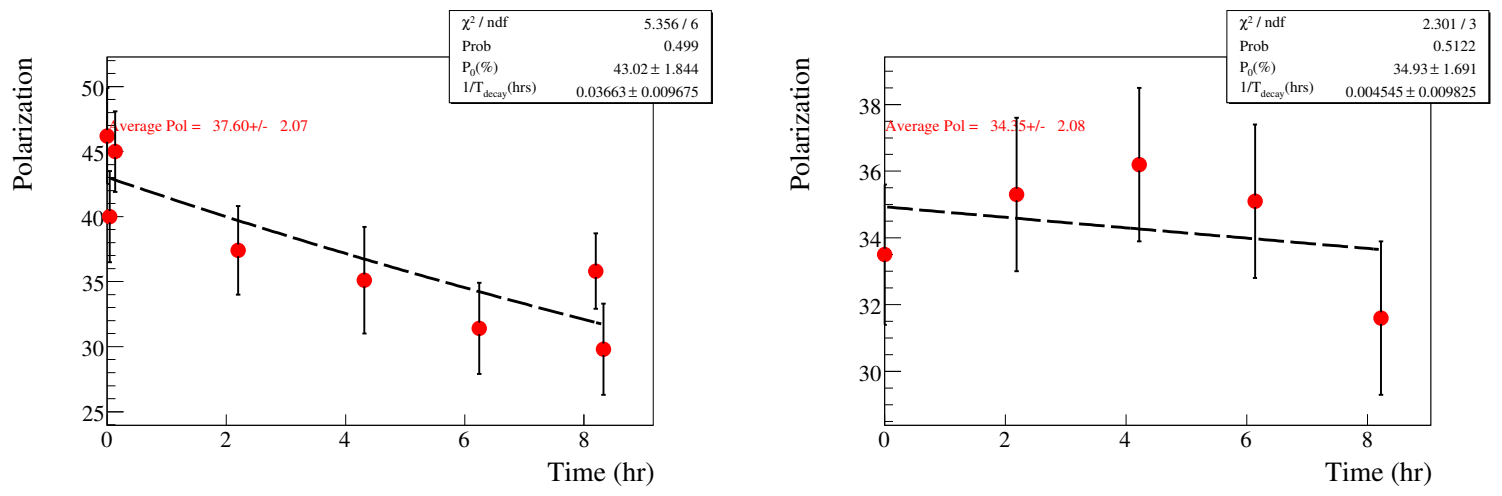

Figure 20: An example of a fill (Yellow fill 9880) that shows large polarization decay (left) and a fill (Yellow fill 9947) that shows relatively small polarization decay (right). Also shown are an exponential fit to data and the average polarization calculated assuming an exponential decay as described in the text.
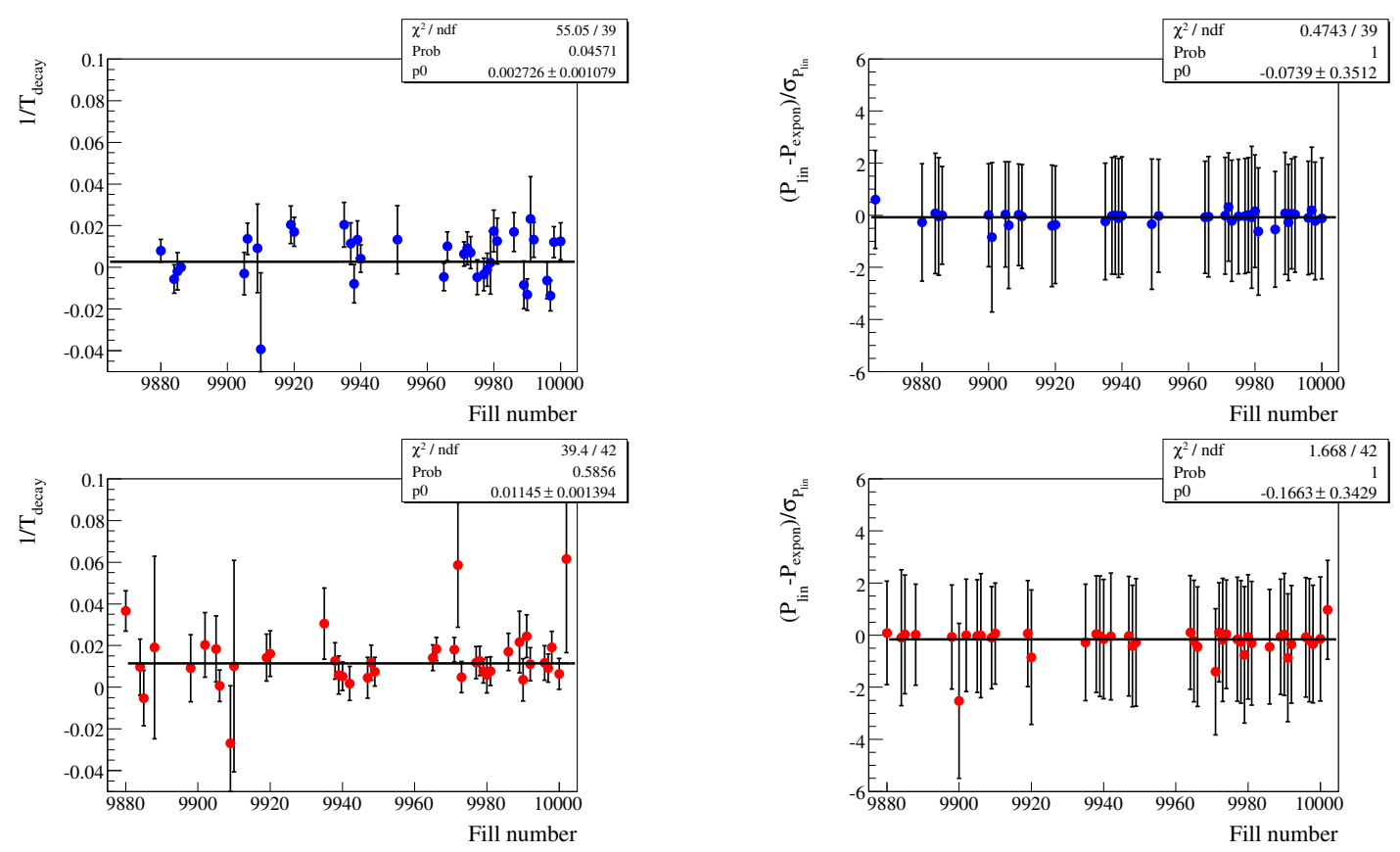

Figure 21: Polarization decay extracted for each fill assuming an exponential decay (top two plots). The difference of the average polarization calculated assuming no decay and an exponential decay is also shown (bottom two plots). See the text for more details. Red and blue markers correspond to yellow and blue beams respectively. 
with unfinished scans that did not reach the maximum intensity $\left(I_{\max }\right)$ as well as runs with unreliable measurements were excluded from calculation of $\mathrm{pC}$ to HJet normalization. A list of runs that were included in the analysis will be given elsewhere [2]. Four blue scan runs and eleven yellow scan runs that are "good" otherwise were not included in the analysis since the scan was unfinished and didn't reach the maximum of the Gaussian profile, as shown in Fig. 22. Tables 1 and 2 and Fig. 23 summarize the normalization constants for blue and yellow beams. Runs that did not have perfect scans and runs did have perfect scans were analyzed separately to determine the normalization constant. An examples of a scan that has perfect profile, scans that did not have perfect profiles but still were included in the analysis, and a run that was not included in the analysis due to incomplete profile are shown in fig. 22. The two normalization constants so obtained did not show any deviation within statistical errors. This confirmed that our technique to obtain average over transverse cross section beam polarization (via fit parameters $P_{\max }$ and $R$ ) is stable.
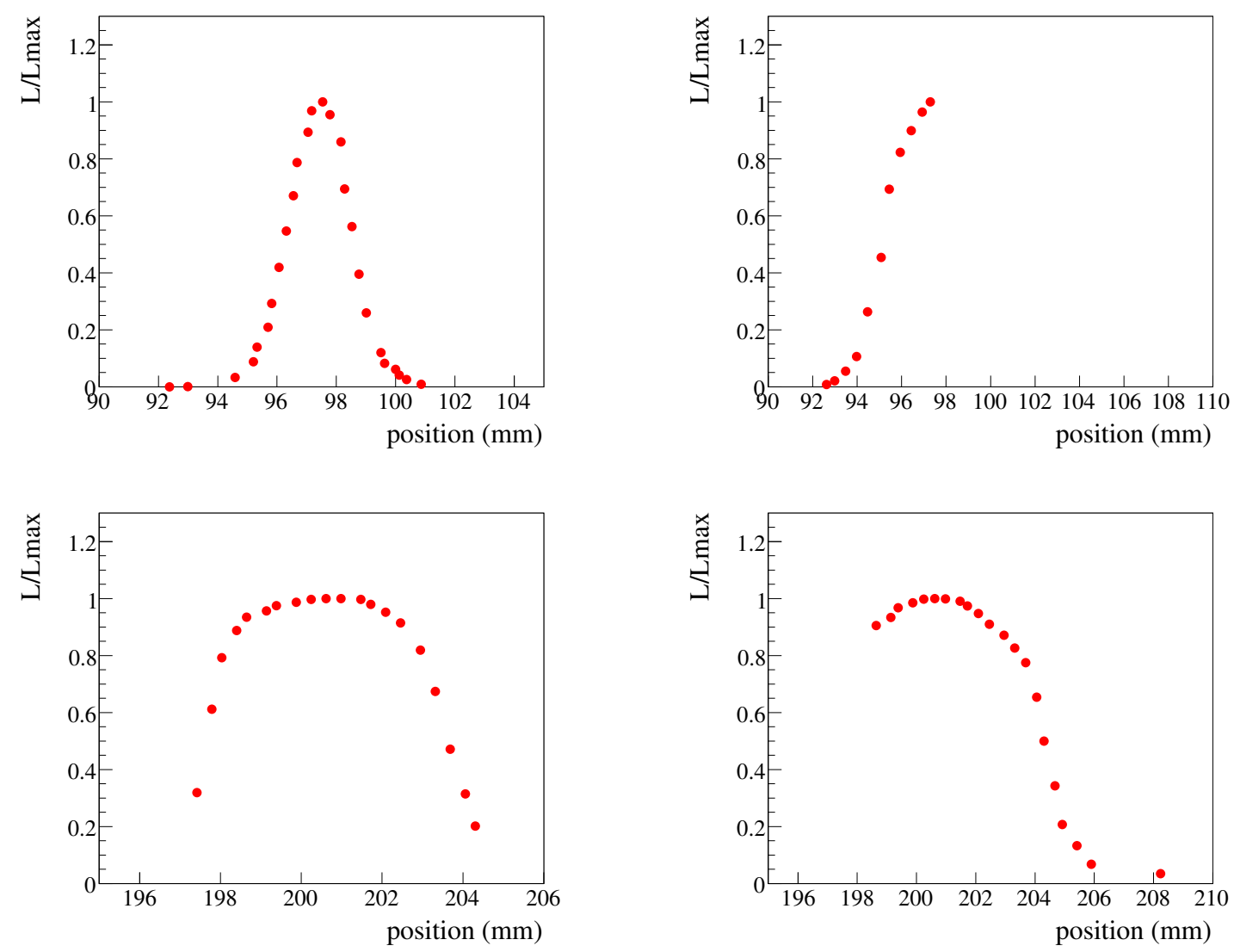

Figure 22: Examples of Luminosity profiles that has the expected Gaussian shape (top left), unfinished scan that was not included in the analysis (top right), and a flat profile and an unfinished scan that were considered acceptable and therefore were included in the analysis (bottom). 
Table 1: Normalization constant pC/HJet (blue) for different periods

\begin{tabular}{|c|c|c|c|c|}
\hline \hline Period & pC average & HJet & $\begin{array}{c}\text { normalization } \\
\text { constant } \\
\text { pC/HJet }\end{array}$ & Fills \\
\hline 1 & $52.02 \pm 0.64$ & $47.93 \pm 2.37$ & $1.086 \pm 0.055$ & $\begin{array}{c}9885,9888,9890, \\
9898,9902,9905, \\
9906,9909\end{array}$ \\
\hline 2 & & & $\begin{array}{c}\text { Did not calculate } \\
\text { (one fill) }\end{array}$ & $\begin{array}{c}9949 \\
\text { (9942 and } 9948 \\
\text { are not included) }\end{array}$ \\
\hline 3 & $47.02 \pm 0.74$ & $43.15 \pm 1.62$ & $1.090 \pm 0.044$ & $\begin{array}{c}9972,9973,9974,9975, \\
9977,9978,9979\end{array}$ \\
\hline 4 & $47.80 \pm 1.38$ & $48.70 \pm 2.43$ & $0.982 \pm 0.057$ & $9991,9992,9996$ \\
\hline ALL & $47.96 \pm 0.53$ & $45.17 \pm 1.11$ & $1.062 \pm 0.029$ & periods 1+2+3+4 \\
\hline \hline
\end{tabular}


Figure 23: Comparison of normalization constant $\mathrm{pC} /$ HJet for different periods as defined in Tables 1 and 2 .

\section{Polarization for experiments}

After normalization for $\mathrm{pC}$ measurements is obtained, the last step is to provide polarization values for experiments which are averages obtained weighting with a prod- 
Table 2: Normalization constant pC/HJet (yellow) for different periods

\begin{tabular}{|c|c|c|c|c|}
\hline \hline Period & pC average & HJet & $\begin{array}{c}\text { normalization } \\
\text { constant } \\
\text { pC/HJet }\end{array}$ & Fills \\
\hline 1 & $41.00 \pm 0.61$ & $39.23 \pm 1.96$ & $1.045 \pm 0.054$ & $\begin{array}{c}9919,9920,9935, \\
9937,9938,9939, \\
9940\end{array}$ \\
\hline 2 & $38.16 \pm 0.73$ & $33.95 \pm 1.97$ & $1.124 \pm 0.069$ & $\begin{array}{c}9951,9965,9966, \\
9971 \\
3\end{array}$ \\
& $39.55 \pm 0.69$ & $35.65 \pm 1.90$ & $1.110 \pm 0.062$ & $\begin{array}{c}9980,9981,9986, \\
9989,9990\end{array}$ \\
\hline ALL & $39.59 \pm 0.39$ & $36.30 \pm 1.12$ & $1.091 \pm 0.035$ & periods $1+2+3$ \\
\hline \hline
\end{tabular}

uct of two beam intensities in both $\mathrm{x}$ and $\mathrm{y}$ transverse dimensions. For the simple case when the transverse size $\left(\sigma_{I}\right)$ is about the same in yellow and blue beams: $<P>=\frac{P_{\max _{2}}}{\sqrt{\left(1+R_{x} / 2\right) \sqrt{\left(1+R_{y} / 2\right)}}}$, where $R_{x}$ and $R_{y}$ are $\left(\frac{\sigma_{I}}{\sigma_{P}}\right)^{2}$ in horizontal and vertical direction respectively, and $P_{\max _{2}}$ - is polarization at the intensity peak in two dimensional

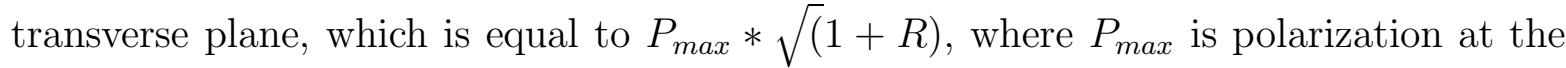
intensity peak in one dimensional case (integrated over the perpendicular direction). Therefor to extract polarization for experiments, the knowledge on polarization profile in both transverse directions is necessary. Since we only have $R$ in either vertical or horizontal direction for each fill, the unmeasured direction was estimated using known values from other fills.

As it is shown on Figs. [16, 17, 25], polarization profile parameter $R$ is about the same for both vertical and horizontal directions. It was decided to use $R=0.15$ for all unmeasured profiles in blue. Possible fill-by-fill variation in $R$ from Fig. 25 of \pm 0.11 (to accommodate $\pm R M S$ in both vertical and horizontal profiles) was used to assign the fill-by-fill uncertainty to polarization measurements, due to unmeasured profile. Here we should mention that the large contributor to RMS of $R$ distributions is statistical fluctuations. Therefore attributing the RMS value to fill by fill variations, will be an overestimation of these variations. The \pm 0.02 uncertainty in the average unmeasured polarization profile (since average $R$ was obtained based on approximately half of the fills and applied to the other half, where it was not measured) is a source of global uncertainty in polarization measurements to be discussed below.

Unfortunately, for the yellow beam only horizontal scans are available. As it is seen in Fig. 26 the $R_{\text {hor }} \pm R M S \sim 0.30 \pm 0.10$. Since we do not have data to determine how sharp the vertical polarization profile for yellow is, we assumed $R_{\text {vert }}$ could possibly vary from 0 to $R_{\text {hor }}+R M S$, which is 0 to 0.40 . Therefore it was decided to use middle 
point $R_{\text {vert }}=0.20$ with possible fill-by-fill variation of \pm 0.20 (which goes to fill-by-fill uncertainty), and with the same uncertainty \pm 0.20 for the average $R_{\text {vert }}$ (which goes to global polarization uncertainty).

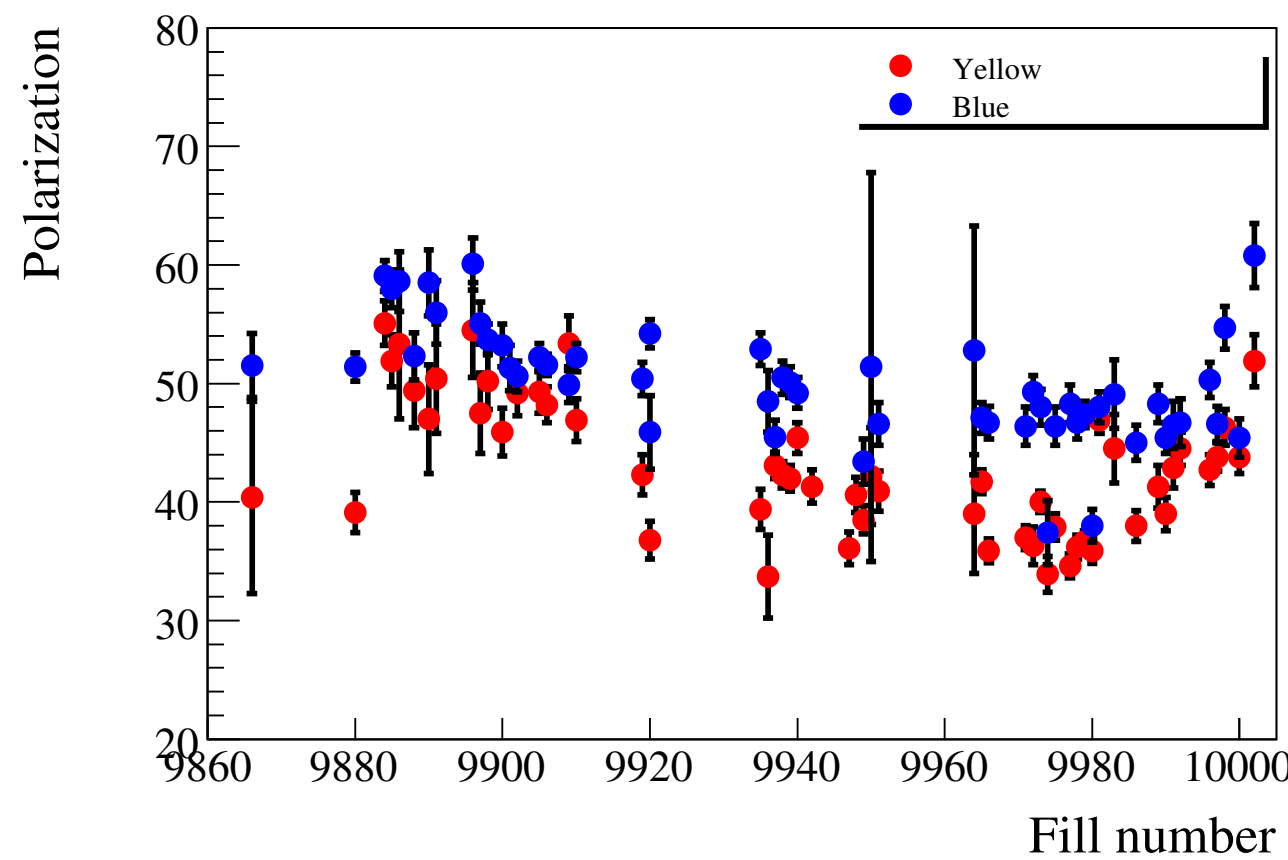

Figure 24: Polarization for experiments calculated as described in the text. Errors only indicate statistical uncertainties.

\section{Systematic uncertainties}

Main systematic uncertainties in pC polarization measurements are due to the uncertainties in polarization profile and the energy correction (dead layer). Some of the uncertainties due to polarization profile are similar to that of run 6 and a similar approach for run 8 was adopted (total 1.1\%):

- In non-continuous scans we may miss the maximum, which might lead to wrong $I_{\text {max }}$; Uncertainty due to this is $\approx 0.2 \%$

- For several runs a flat luminosity profile, instead of a Gaussian peak was observed. One of the possible reasons could be target vibrations, giving average over a certain distance rather than a measurement at one fixed place across the beam. A simulation was performed to determine uncertainties due to this [3]. And the upper limit for this contribution to polarization measurements was determined to be $\approx 1 \%$. 
- An uncertainty of $0.5 \%$ was assigned to take into account the assumption that the profiles are Gaussian.

The main uncertainty due to polarization profile arises due to variation in $R$ and also due to having the profile information for either horizontal or vertical profile. As we discussed in previous section the $\pm 0.11( \pm 0.20)$ fill by fill possible fluctuations in unmeasured polarization profile in blue (yellow) beams lead to $\sim 3 \%(\sim 5 \%)$ uncertainty in polarization measurements for experiments, in which the parameter $R$ enters as $1 / \sqrt{1+R / 2}$. The uncertainties for average $\mathrm{R}$ (over all measurements) for blue (yellow) beams are $\pm 0.02( \pm 0.20)$. In addition, $R$ for unmeasured profile lead to a global uncertainty of $\sim 0.5 \%(\sim 5 \%)$ in polarizations for experiments.
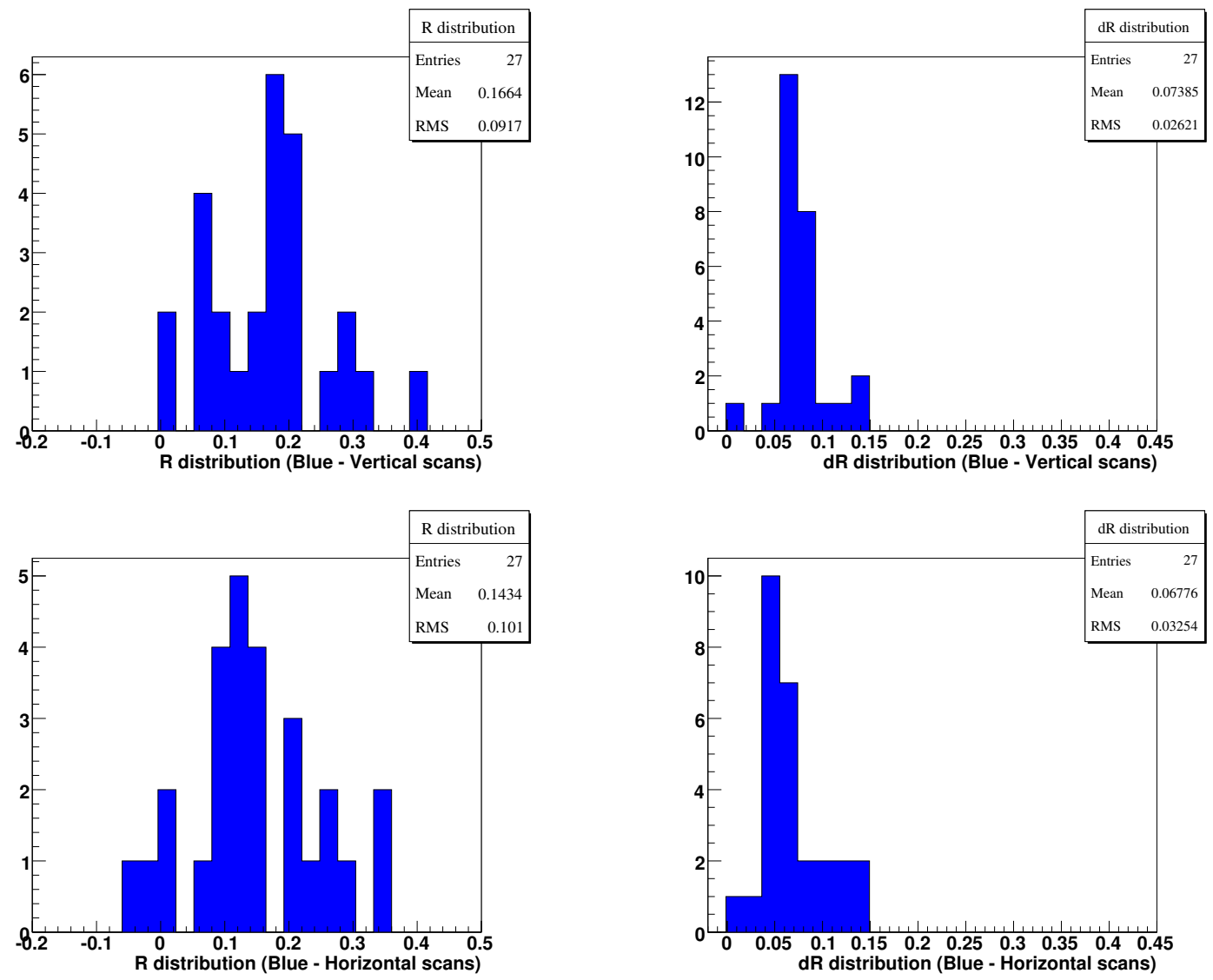

Figure 25: Distribution of $R$ and the errors of $R$ for blue vertical (top plots) and blue horizontal (bottom) scans.

The other systematic uncertainty as described above is due to the fill to fill variation of the energy correction (dead layer). Fig. 27 shows the dead layer history for all scan measurements that were included in the analysis. On average the energy correction for the blue (yellow) detectors varied $\pm 3 \mu \mathrm{g} / \mathrm{cm}^{2}\left( \pm 4 \mu \mathrm{g} / \mathrm{cm}^{2}\right)$ from the average, that corresponds to $1.8 \%(2.4 \%)$ uncertainty. This is based on an average $0.6 \%$ effect on 

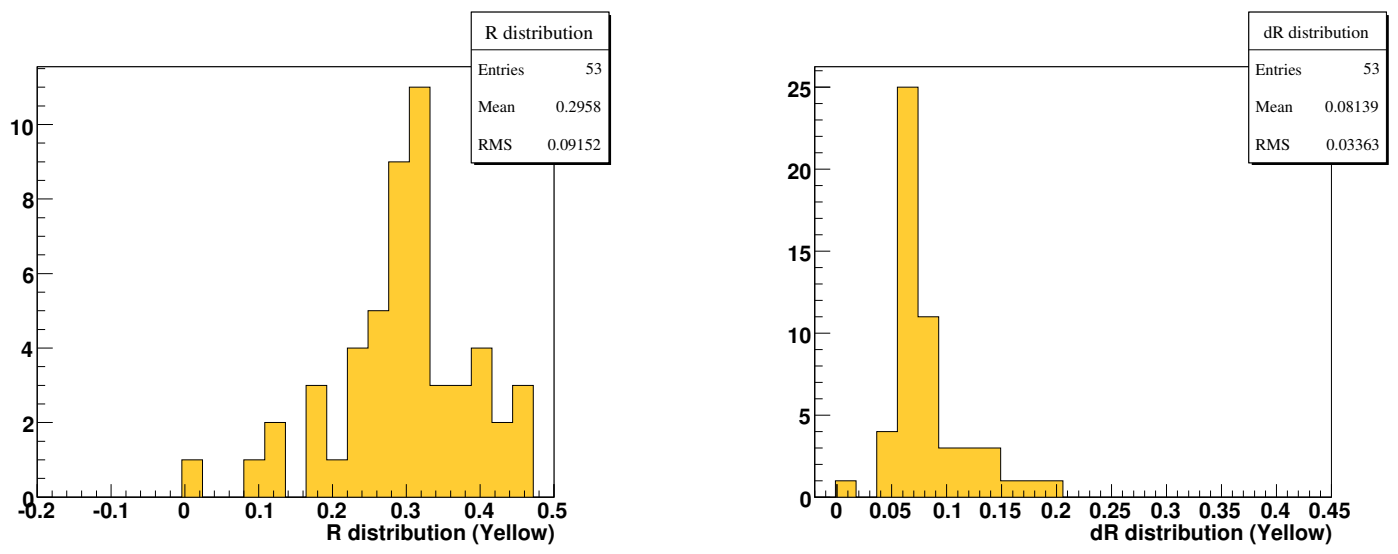

Figure 26: Distribution of $R$ and the errors of $R$ for yellow horizontal scans.

polarization from $1 \mu \mathrm{g} / \mathrm{cm}^{2}$ change in DL. Assigning these global uncertainties assumes our poor knowledge on why the dead layer drifts over time, therefore a $100 \%$ uncertainty was given for those corrections. Deviation of the dead layer correction from a linear fit to dead layer Vs fill number was used to determine the fill to fill uncertainties. Fig. 28 shows the distribution of the deviation. The RMS values of the distributions $\left(1.6 \mu \mathrm{g} / \mathrm{cm}^{2}\right.$ and $1.1 \mu \mathrm{g} / \mathrm{cm}^{2}$ ) were used to assign errors, which are $1 \%$ and $0.7 \%$ for blue and yellow respectively. Table 3 and 4 summarize the final systematic uncertainties.
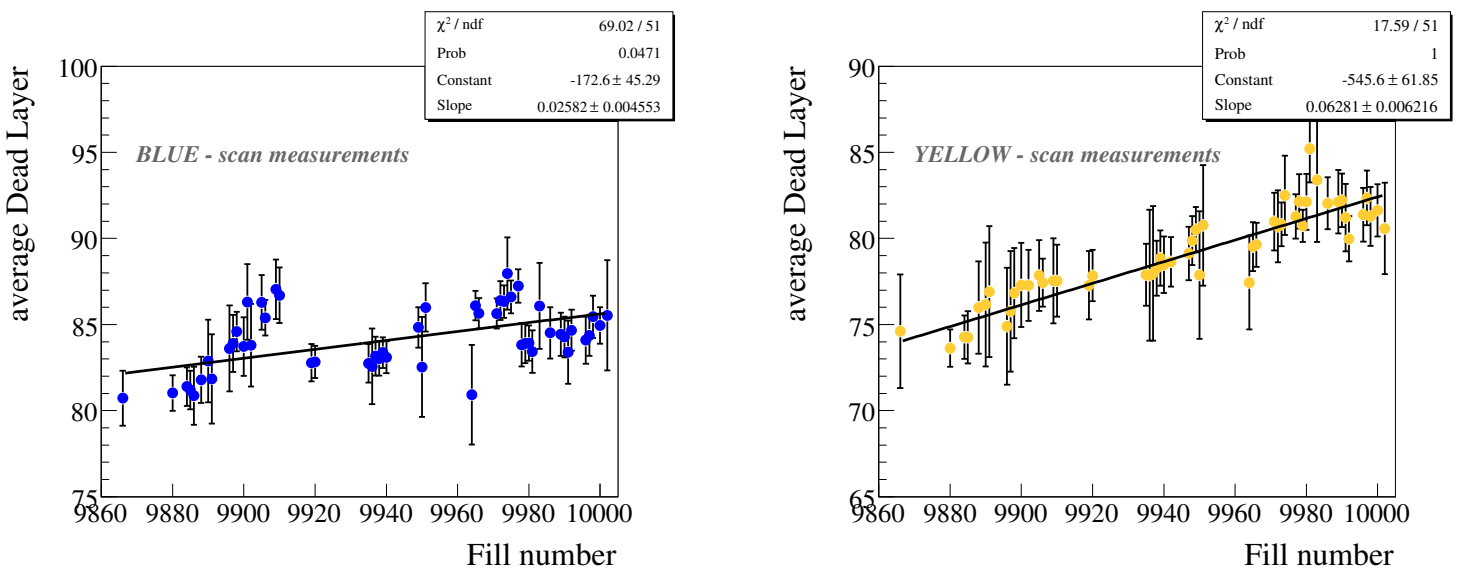

Figure 27: Dead layer (averaged over fills) history of all the scan measurements that were included in the analysis.

The final global uncertainties, deltaP/P, are:

Blue: $4.2 \%$

Yellow: $7.2 \%$

Considering that "Jet normalization, systematic" as well as "Energy correction" uncertainties are mostly correlated between blue and yellow, the final global uncertainties for 

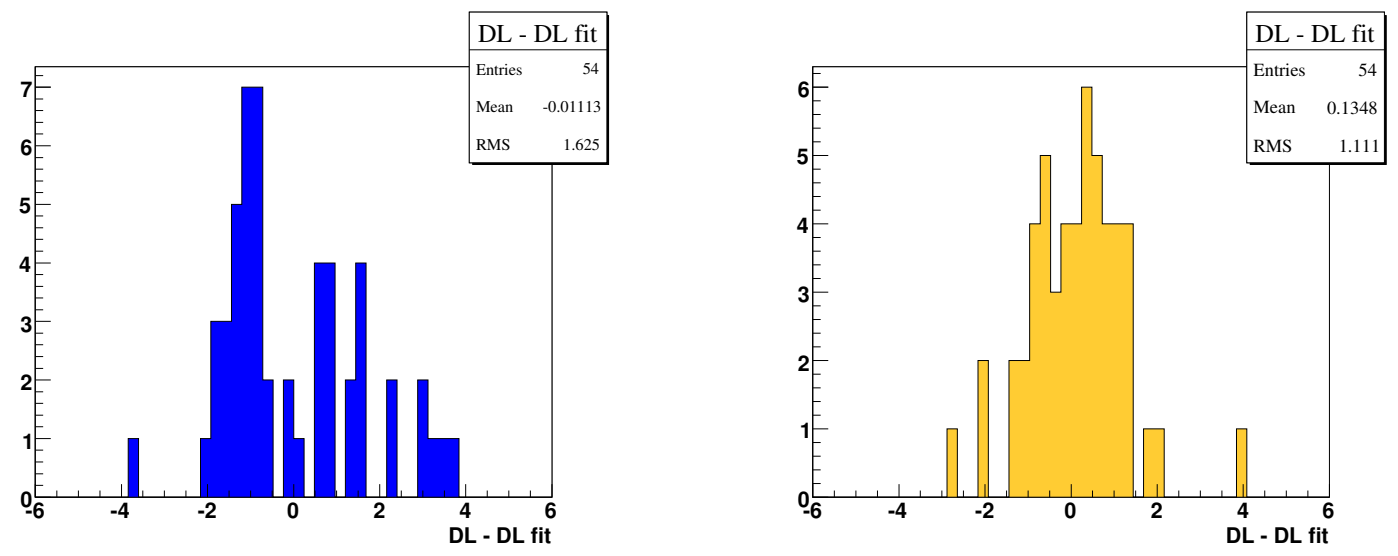

Figure 28: Distribution of the deviation of dead layer from a fit (as shown in Fig. 27 to dead layer history.

Table 3: fill-by-fill (non-correlated) systematic uncertainties

\begin{tabular}{ccc}
\hline \hline & & \\
& Blue & Yellow \\
\hline From vert/horizontal profile & $3 \%$ & $5 \%$ \\
Energy correction & $1 \%$ & $0.7 \%$ \\
\hline \hline
\end{tabular}

a product of two beams, $\delta\left(P_{B} * P_{Y}\right) /\left(P_{B} * P_{Y}\right): 9.6 \%$

\section{References}

[1] I. Nakagawa et al., RHIC/CAD Accelerator Physics Note Vol.275 (2007)

[2] pC Run-8 run summary

[3] A. Bazilevsky, analysis of Run $6 \mathrm{pC}$ systematic errors http://www4.rcf.bnl.gov/ cnipol/pubdocs/Run060ffline/RSC_30nov2007_sasha.ppt RHIC/CAD Accelerator Physics Note 298 
Table 4: Global systematic uncertainties (correlated from fill to fill)

\begin{tabular}{ccc}
\hline \hline & & \\
& Blue & Yellow \\
\hline Jet normalization, stat & $2.7 \%$ & $3.2 \%$ \\
Jet normalization (profile) & $1.1 \%$ & $1.1 \%$ \\
Jet normalization, syst (molecular) & $2.0 \%$ & $2.0 \%$ \\
Jet normalization, syst (other) & $1.3 \%$ & $2.4 \%$ \\
Pol. profile (vert. for exp) & $0.5 \%$ & $5.0 \%$ \\
Energy correction: & $1.8 \%$ & $2.4 \%$ \\
\hline \hline
\end{tabular}




\title{
Run-08 pC polarization analysis
}

\section{October 16, 2008}

\author{
V. Dharmawardane \\ New Mexico State University, Las Cruces, NM 88003 \\ A. Bazilevsky, G. Bunce, R. Gill, H. Huang, Y. Makdisi, \\ I. Nakagawa, B. Morozov, H. Okada, M. Sivertz, A. Zelenski \\ Brookhaven National Lab, Upton, NY 11973 \\ I. Alekseev, D. Svirida \\ Institute of Theoretical and Experimental Physics, 117259, Moscow, Russia
}




\section{Introduction}

In this note we will discuss the analysis of RHIC run $08 \mathrm{pC}$ data that were collected during February 14 - March 10, 2008. An analysis method that is similar to Run 05 and Run 06 was adopted for Run 08 analysis (except few minor changes, which are described below). A detailed analysis note and a NIM article that describe the $\mathrm{pC}$ analysis procedure (for run 05 and run 06) can be found elsewhere [1]. In brief, the analysis consists of calibrating the detectors, determining energy corrections ("dead layers"), determining good runs and extracting the polarization from data. Next few sections describe these steps more in detail.

\section{Calibrations}

Each silicon detector used to detect recoil carbon ions are segmented into 12 strips. In total, the pC polarimeter has 6 such detectors mounted at 45, 90, 135 degrees in both left and right sides perpendicular to the beam direction. The silicon Strip Detector (SSD) have a thickness of about $400 \mu \mathrm{m}$, which is sufficient to stop recoil carbon ions of energies up to about $100 \mathrm{MeV}$. Each silicon detector consists of n type bulk material, with a surplus of electron sites in the crystal lattice. The backplane has an aluminum contact over the complete surface. The front face has approximately $150 \mathrm{~nm}$ deep p type silicon strips implanted in the surface. When a charged particle passes through the silicon detector it creates electron-hole pairs in the semiconductor wafer. These electronhole pairs are drifted toward backplane and the surface respectively due to the electric field. The current is measured by small aluminum strips on the surface of each strip.

\subsection{Energy calibration}

Alpha particle sources mounted inside the polarimeter vacuum were used for calibrating the silicon strip detectors. During run 8, four such measurements were taken periodically to account for changes in the detector response. Figs 1 and 2 show calibration constants for both Blue and Yellow detectors. The constants remained relatively stable throughout the run for Blue detectors. However, for the Yellow detector, approximately $15 \%$ change in constants were observed for detector 5 . The exact fill/time at which this occurred was determined by looking at the dead layer values (which will be discussed next). This was possible because any shift in gains will immediately lead to a shift in extracted "dead layers", if wrong calibration constants that do not reflect the proper shift in gain are used. The same could be obtained by fixing "dead layer" values and checking recoil carbon mass. It was determined that the change occurred on February 16 th 2008 that corresponds to Fill 9886. These findings coincide with observation of a jump in detector 5 current around the same time. Therefore, it was decided to use calibration constants extracted using the analysis of data taken on February 13 th until February 16th (Fill 9886) and constants from data taken on February 27 th for runs taken after-wards. 


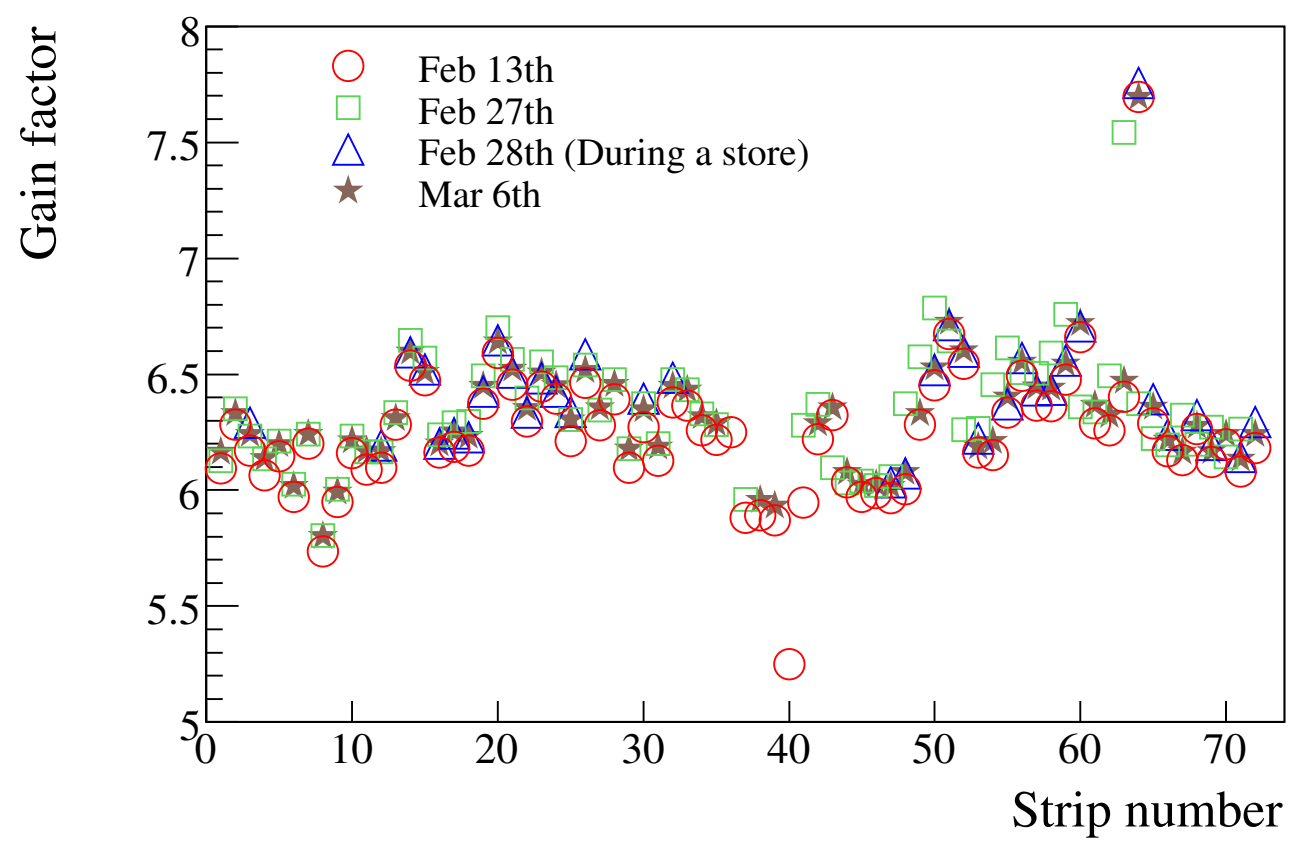

Figure 1: Gain factor as a function of strip number for blue detectors.

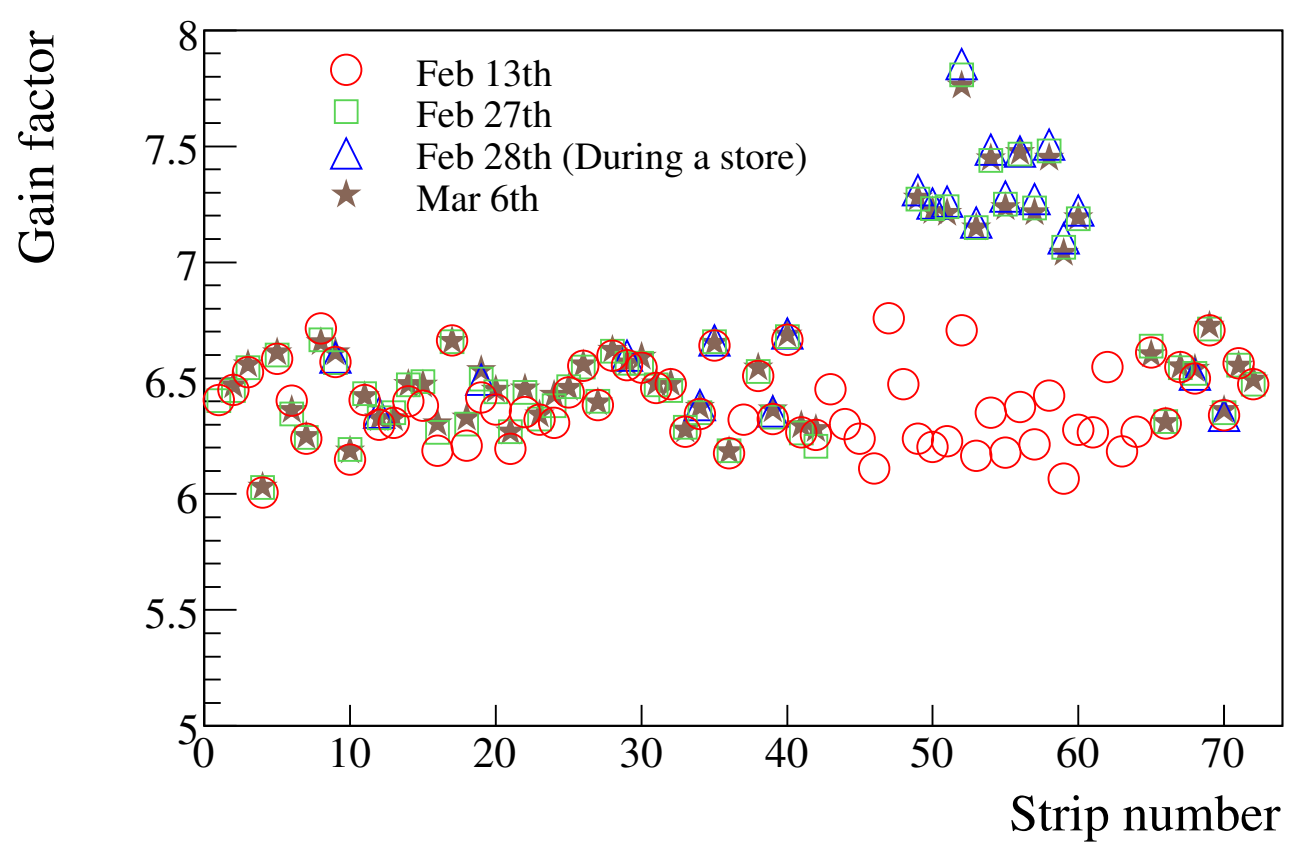

Figure 2: Gain factor as a function of strip number for yellow detectors 


\subsection{Dead layer and t0}

The next step of pC calibration consists of extracting t0, a ToF offset, and dead layer (DL) for each strip in each measurement (run). The $\mathrm{p}+$ doping layer described above is called the "dead layer". The energy lost in this dead layer cannot be measured and is energy dependent and can be described by a known function of energy [1]. Since the $\alpha$ calibration does not effectively probe this surface region of the detectors additional corrections are needed to accurately reconstruct the energy of scattered carbons. These calibration constants were extracted by doing a fit to the "banana" plot, which is the recoil Carbon time-of-flight ( $\mathrm{ToF}$ ) vs energy. The DL parameter carries the meaning of "effective" dead layer and is used to correct the carbon deposited energy to obtain carbon kinetic energy. For run 5 and run 6 the fit was performed for kinetic energy range 400-900 keV. However for run 8 this has to be tightened, since beam-induced pulses caused problems in the polarimetry operation. Fig. 3 shows an example of noise from beam-induced pulses for one run. To remove this background from the data stream

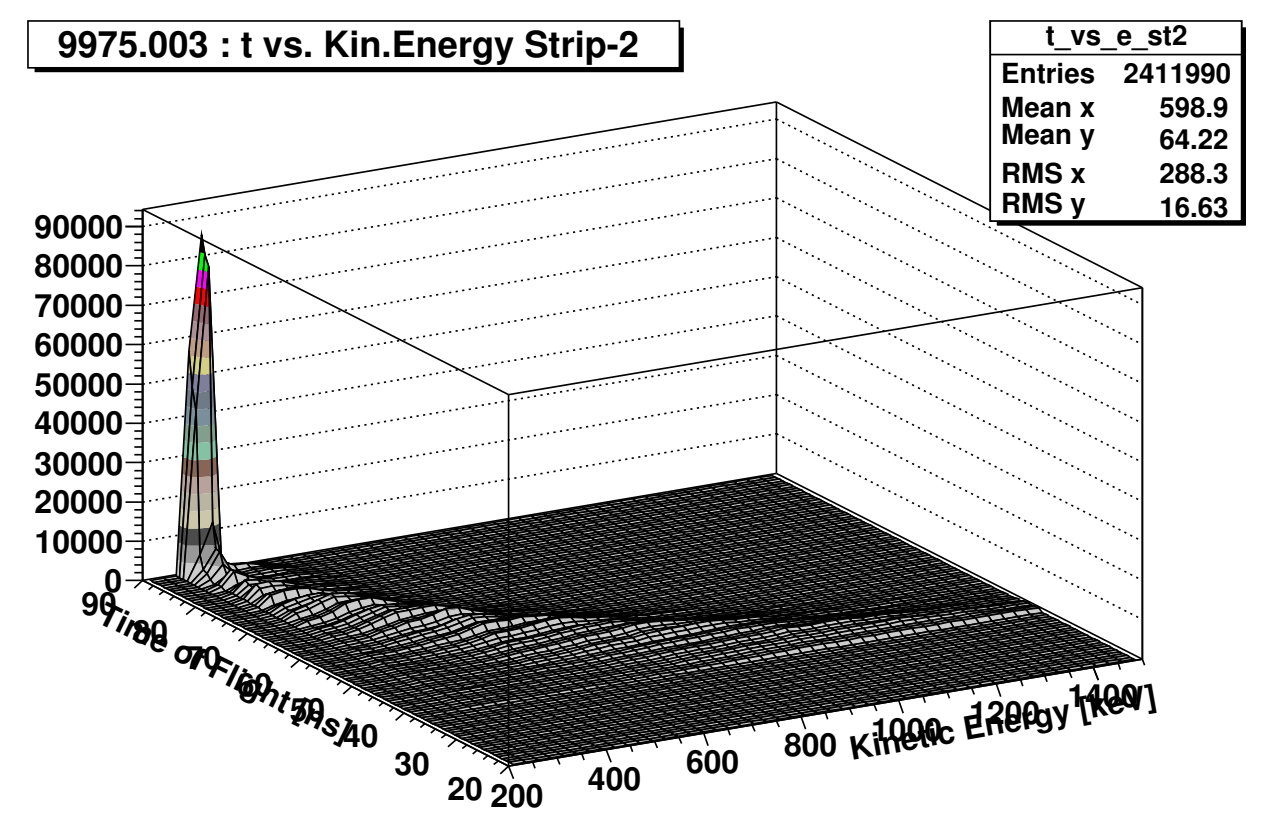

Figure 3: Time of flight vs kinetic energy for a run. Large peak seen at small kinetic energy is due to noise from beam-induced pulses.

time window had to be tightened starting fill 9978, which caused an asymmetric shape in the kinetic energy Vs time distribution. Fig. 4 shows an examples of time of flight Vs kinetic energy distribution before the time window was tightened and after the time window was tightened. Dead layer calibration assumes a symmetric shape in the time Vs kinetic energy distribution. Therefor runs taken starting fill 9978 were calibrated using a kinetic energy cut of 500-900 keV. To ensure that events due to beam induced pulses that may have leaked into the data stream are not included in the analysis, it was also 

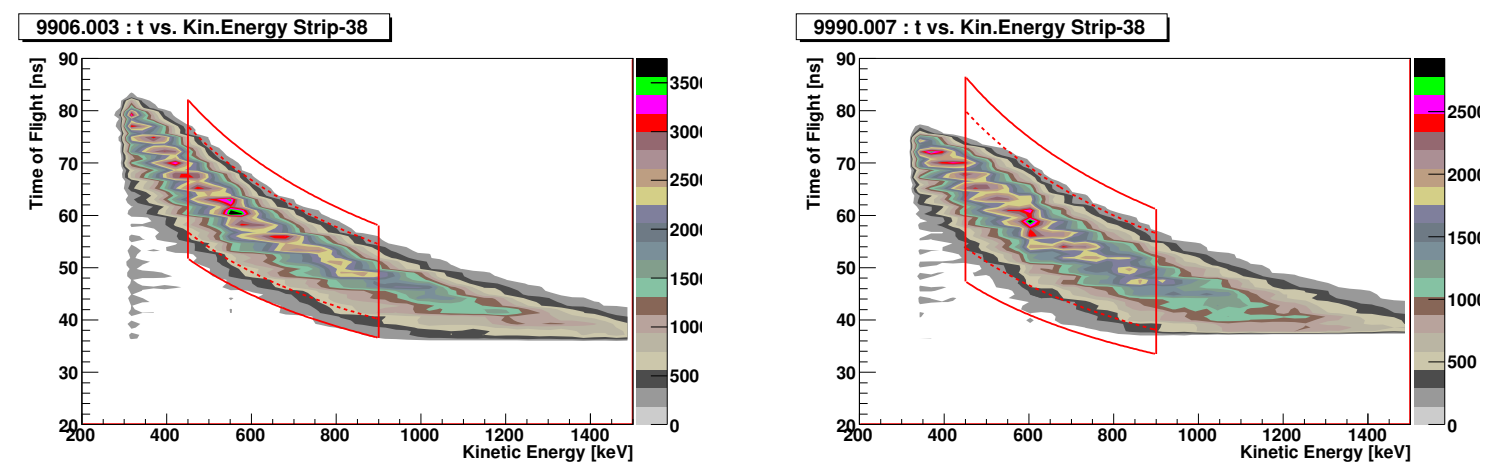

Figure 4: Time of flight Vs kinetic energy distribution before the time window was tightened (left) and after the time window was tightened (right). Asymmetric shape of the banana at low kinetic energy is clearly visible on the right plot.

decided to use a 450-900 keV energy cut instead of the same cut as in run 5 and 6 (400$900 \mathrm{keV}$ ) for runs taken before run 9978. Several studies were performed to determine whether using different cuts for different periods have any significant systematic effect on the relative polarization. It was concluded that using a different cut for dead layer calibration does not cause any significant systematic effect compared to the runs that were calibrated using a 450-900 keV energy cut. A more detailed description of the dead layer and the t0 calibration procedure can be found at [1]. Figs. 5 and 6 show the Run 8 dead layer history for each detector and Fig. 7 shows the average $T 0$ history for both blue and yellow detectors.

\section{Data quality}

The list of QA checks was the same as in Run5 and Run6 pC analysis. It included control of the width and position of the carbon (C) mass peak, as well as $\mathrm{C}$ mass peak position vs its kinetic energy (which detects problems with WFD and/or DAQ and/or in the fit of "banana"; strip by strip variations of the number of events in the "banana"; consistency in bunch-by-bunch asymmetry measurements. All systematic uncertainties from the effects above were estimated to be negligible for the final fill-by-fill polarization measurements, except the energy correction effect (described by DL), which will be described more in detail in the section systematic uncertainties. In general following criteria were used to determine good/bad strips/runs. However, when the deviations are marginal they were considered as good.

- C mass position: Strips with $0.5 \mathrm{GeV}$ deviation from the Carbon mass were not included in the analysis.

- Number of events in banana: Strips outside $20 \%$ from the average were removed. 

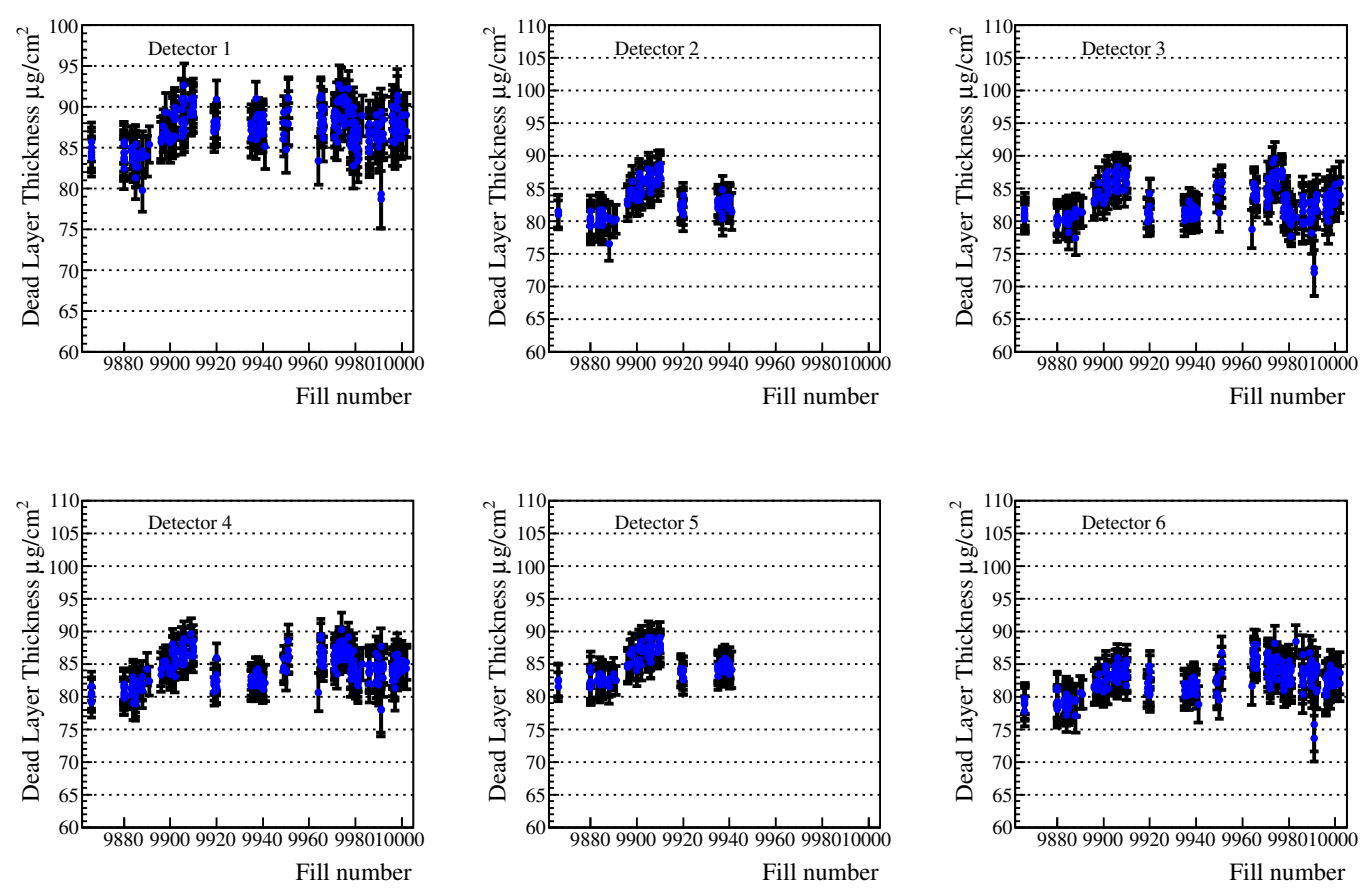

Figure 5: Dead layer history for blue detectors.

- C mass width: strips with a deviation above 1.92 sigmas were not included in the analysis.

- Specific luminosity per bunch: Specific luminosity is defined by the ratio between number of events per bunch within kinematic cuts and the intensity per bunch measured by the wall current monitor. The number of events are summed for all active strips for a given bunch. Under perfect conditions specific luminosity per bunch should be a constant for all bunches. However, runs taken with the blue vertical target 5 (Fill numbers : 9942, 9947, 9948 and 9949) showed large deviations from the average for first few bunches. An example of one such run is given in Fig. 9. These runs were taken with a thick target in fills with high beam intensity, leading to extremely high event rates ( $5 \mathrm{MHz}$ for events within banana). The problem might be connected with base line shift in WFDs. However, we didn't find a way to correct this effect, and it was decided not to include any of those runs in the analysis and to borrow results from the HJet analysis for those fills. Unfortunately, we do not have any HJet measurement for fill 9947, therefore we cannot provide any polarization measurement for this fill. For Fill 9949 few runs were taken with a different target, therefore $\mathrm{pC}$ polarization results for this fill can be provided. 



Figure 6: Dead layer history for yellow detectors.
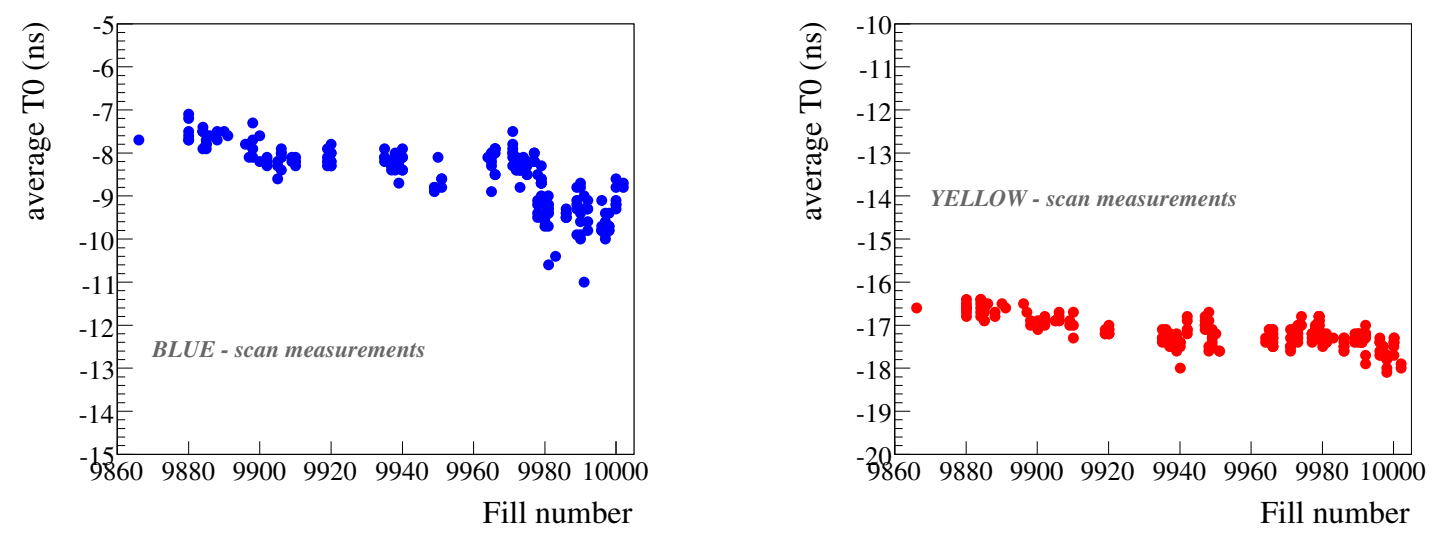

Figure 7: T0 history for blue and yellow detectors.

On total two blue strips and five yellow strips were permanently disabled. Four yellow strips showed a secondary peak near the carbon peak due to electronic jitter and were permanently disabled. One yellow strip was electronically disconnected and was also permanently disabled. The two blue strips that were permanently disabled fell outside the criteria defined for "good quality" throughout the run. In addition many strips were disabled temporarily (in some case just one run and sometimes for several runs in a 
row) for some runs and they were enabled once the strips turned back to normal and fell within the criteria defined above for "good" strips. On maximum, 8 strips (per run) were disabled for some runs. A list of runs that were not included in the analysis due to problems (such as hardware failure and mechanical problems that prevented from making a proper target scan measurement) that cannot be recovered by disabling strips is given elsewhere [2]. However, most of the runs that were not included are simply "Junk" runs or runs with very few or zero statistics and therefore could not be analyzed. Four blue and eleven yellow scan runs that are good otherwise were removed from the analysis due to bad scans (an example of a bad scan is given in Fig. 22. Details regarding scan data will be discussed more in detail later in the text)
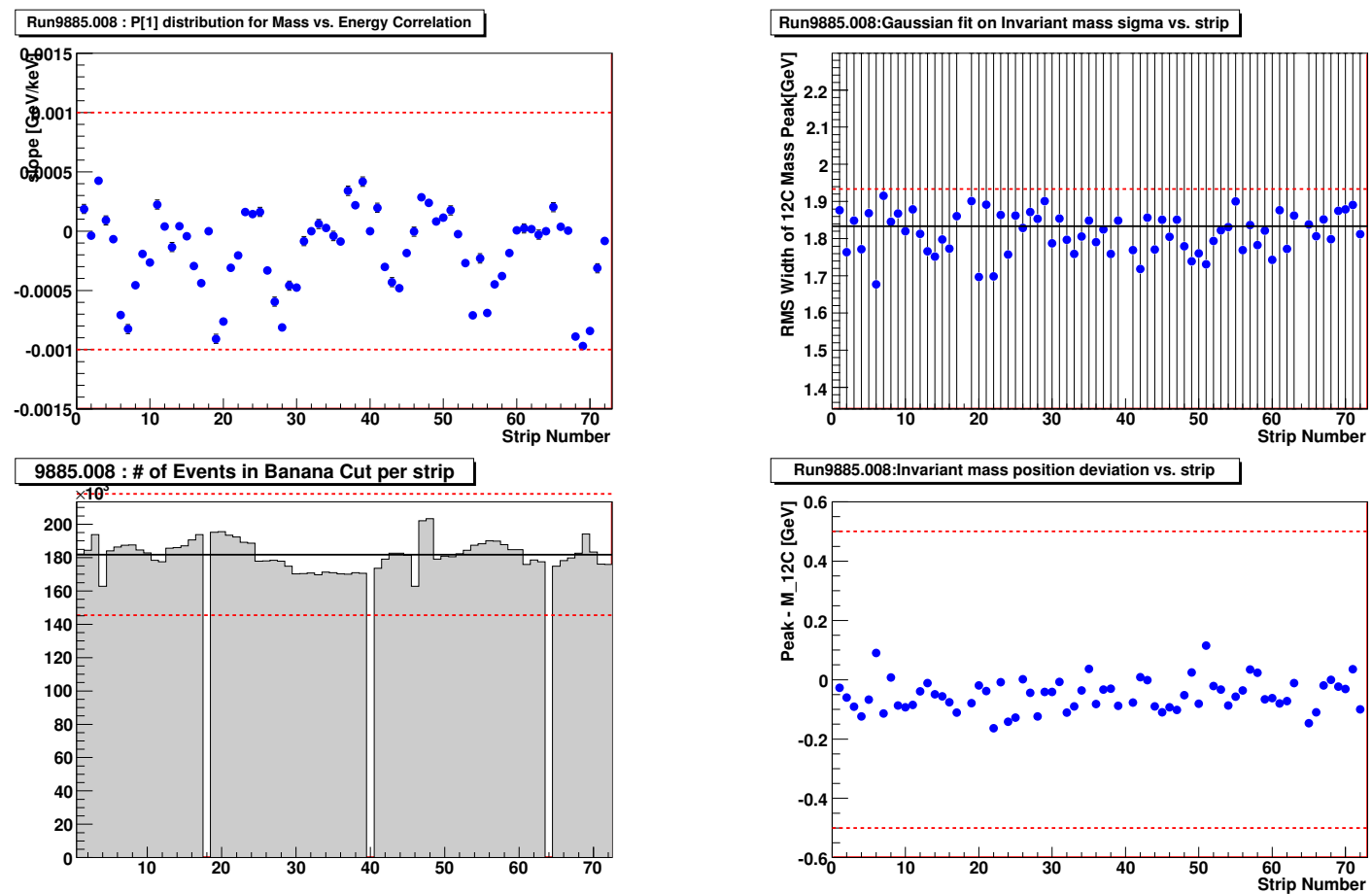

Figure 8: An example of quality checks for a typical run that was used in the analysis.

\section{Polarization profile}

For run 08 , pC polarimeter data were taken with the scan mode and fixed target mode. In the scan mode, measurements were performed with vertical targets and horizontal targets, stepping, in x (transverse horizontal coordinate) or y (vertical coordinate) across the beam, with equal measurement time at each step. In latter part of Run8 we used continuous scan mode in the measurements, instead of step wise. It allowed to measure horizontal/vertical polarization profile in each run separately (with limited statistical precision). All the Yellow data were taken with the Vertical targets, which provided horizontal polarization profile measurements. Blue data were taken with both Horizontal 


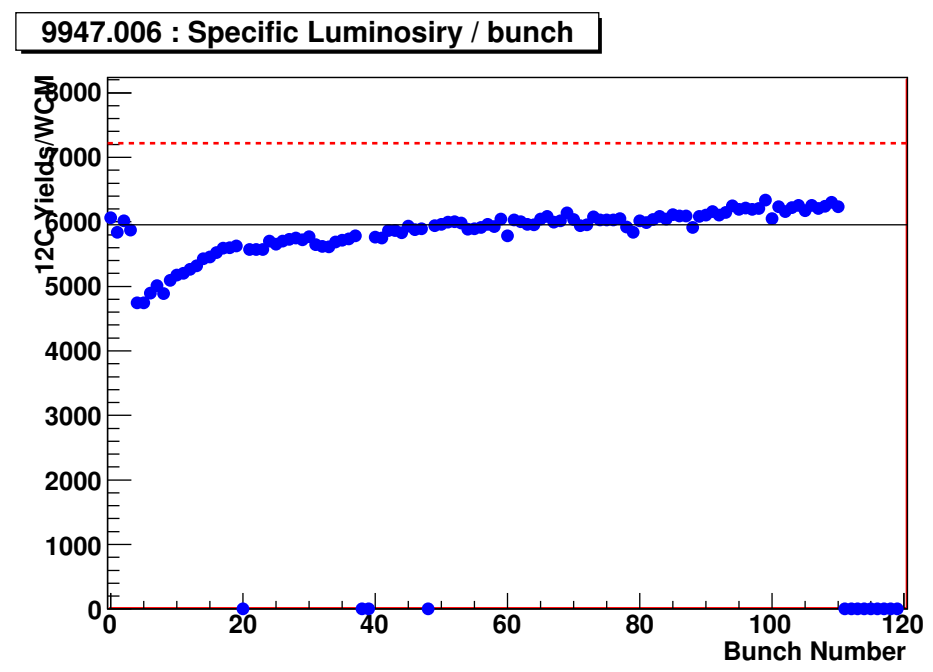

Figure 9: An example of Carbon yield/ wall current monitor as a function of bunch number for a run that was not included in the analysis.

and Vertical targets. Typically, fixed target measurements were made at the beginning and at the end of each fill with the target positioned at the peak intensity in $\mathrm{x}$ or $\mathrm{y}$, and scan measurements were made approximately once per every two hours at flattop energy. In the scan mode data taken at each target position is analyzed exactly the same way the data are analyzed for the fixed target mode. In general, relative polarization $\left(P_{0}\right)$ and the phase factor phi $\left(\phi_{0}\right)$ are determined by doing a fit that is of the form $P_{0}\left(\sin \left(\phi+\phi_{0}\right)\right)$ to measured asymmetries (Fig 10). For run 8 a similar procedure as run 6 was followed in the analysis of scan data except how the phase factor $\phi_{0}$ is determined for each scan target position. In the analysis of run 6 data $\phi_{0}$ was not kept fixed for all target positions. For run 8 analysis $\phi_{0}$ at each target position was kept fixed at the $\phi_{0}$ obtained by doing a fit to all scan data of that run. Fig 11 compares the effect on the relative polarization extracted using the two methods. As expected this new procedure didn't change the extracted polarization much for target positions with plenty of statistics. However for positions with fewer statistics, a few percent change in the extracted polarization was observed, and in general showed a trend towards a slightly lower polarization, leading to a sharper polarization profile. This effect was confirmed in a simple MC: keeping $\phi_{0}$ parameter free for all scan positions (Run6 approach) leads to a bias in polarization measurements (to higher values) when statistics are low. Fig. 12 shows the average $\phi_{0}$ for each fill for all Run 8 scan measurements.

Similar to run 5 and run 6 , the strategy is to obtain the normalization for pC measurements using absolute polarization measurements with HJet in the fills for which HJet measurement is available, and after that use the properly normalized pC measurements to define the polarization in each physics fill.

To obtain average polarization over the beam intensity distribution in the transverse plane, the knowledge on the polarization profile (polarization vs $\mathrm{x}$ and $\mathrm{y}$ in transverse plane) is necessary. The correction due to polarization profile depends on the ratio of 


\section{Run9935.001: Strip Asymmetry sin(phi) fit}



Figure 10: An example of a fit to asymmetries. Polarization and the "phase factor", $\phi_{0}$, are extracted by doing a fit of the form $P_{0}\left(\sin \left(\phi+\phi_{0}\right)\right)$.

width of the beam intensity profile and beam polarization profile. It can be obtained from the direct measurements of the widths of the profiles, which requires good target positioning during the scan. Another way, which excludes the necessity of the precise target positioning, is a fit of polarization vs event rate (which is proportional to beam intensity) in a scan, by a function $\frac{P}{P_{\max }}=\left(\frac{I}{I_{\max }}\right)^{R}$; here it is assumed that both intensity and polarization profiles have Gaussian shapes with widths $\sigma_{I}$ and $\sigma_{P}$, correspondingly, and at least one point in the scan corresponds to beam maximum intensity; $P_{\max }$ and $I_{\max }$ are polarization and event rate at beam maximum intensity; $R$ is $\left(\frac{\sigma_{I}}{\sigma_{P}}\right)^{2}$. Since many of the $\mathrm{pC}$ measurements showed non-Gaussian intensity profiles, which may be due to target positioning problems, this latter approach was used to extract $P_{\max }$ and $R$ parameters for each fill (Fig 13), which were used to calculate the average beam polarization (when doing normalization to HJet measurements) and for colliding beams: $<P>=\frac{P_{\max }}{\sqrt{(1+R)}}$ and $\langle P\rangle=\frac{P_{\max }}{\sqrt{(1+R / 2)}}$, correspondingly, for one dimensional case. Figs.14 - 18 show $R$ and $P_{\max }$ for all blue and yellow scan data for run 8 as a function of the fill number.

As a consistency check average polarization calculated using $P_{\max }$ and $R$ were compared to the average polarization extracted by simply combining the data taken at each scan position. The average polarization for each fill in the latter case was determined by taking the weighted average of all average polarizations obtained for each run. This will ensure that the assumptions that were made in extracting the fit parameters are correct. 


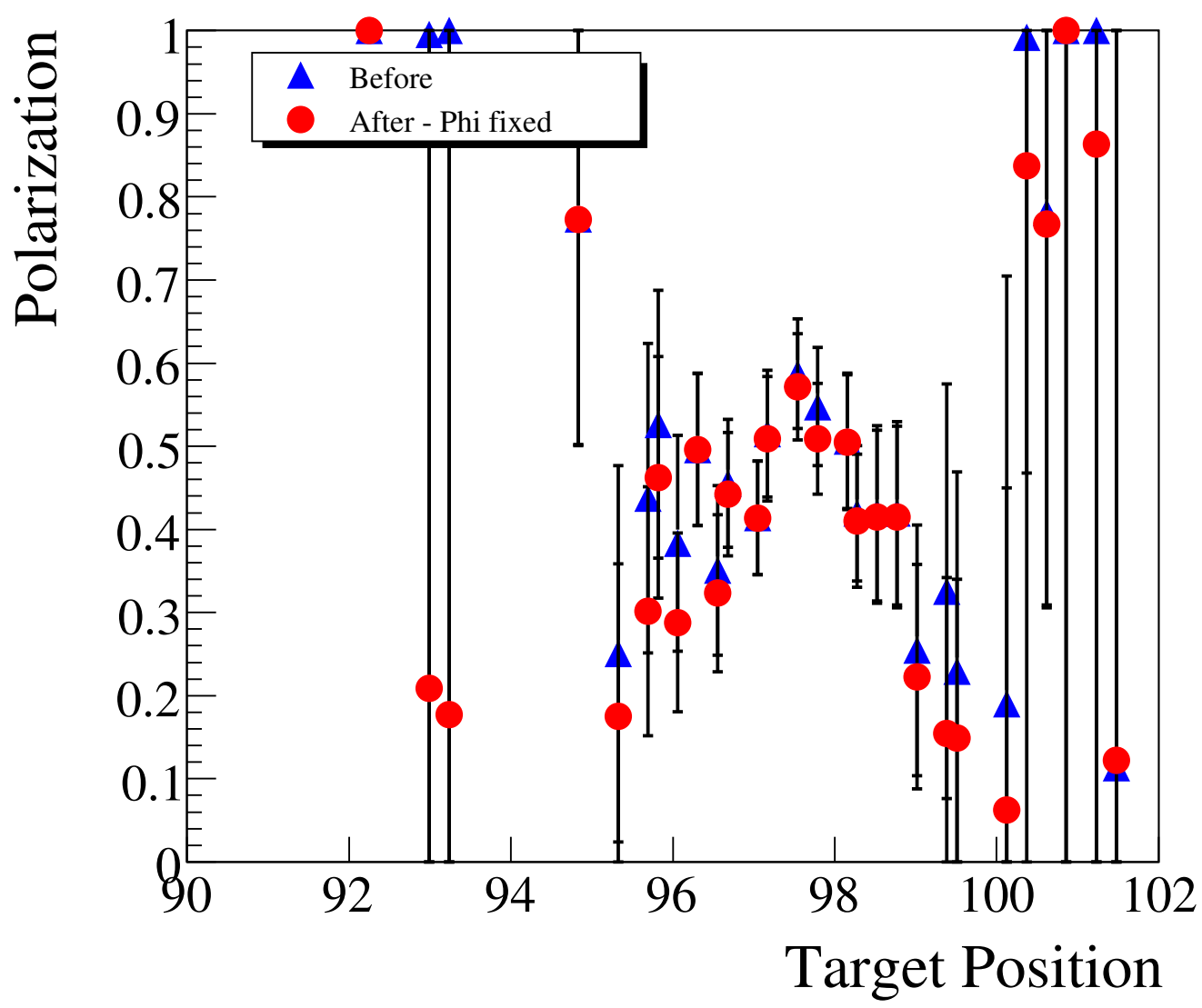

Figure 11: Comparison of polarization extracted by keeping $\phi_{0}$ fixed and letting it vary. More details can be found in the text.

Fig. 19 compares the average polarization calculated using the two methods and the difference in the polarization for the two methods. The difference is approximately 1.4 $\pm 0.3 \%$ and $-0.7 \pm 0.6 \%$ for blue and yellow beams respectively. Slight difference in blue is explained by flatter (non-Gaussian) intensity profiles seen in each scan measurements, which means that effectively during the measurements target didn't move uniformly, but on the average stayed longer near beam center, which led to higher average polarization over a run (so led to a bias in polarization measurements).

\section{Polarization decay}

When combining runs we assume that the polarization decay within a fill is negligible. To make sure that this assumption is true polarization decay for each fill was studied. First, no polarization decay was assumed and a linear fit to polarization was performed to obtain the average polarization. In the case of any polarization decay, an exponential decay 


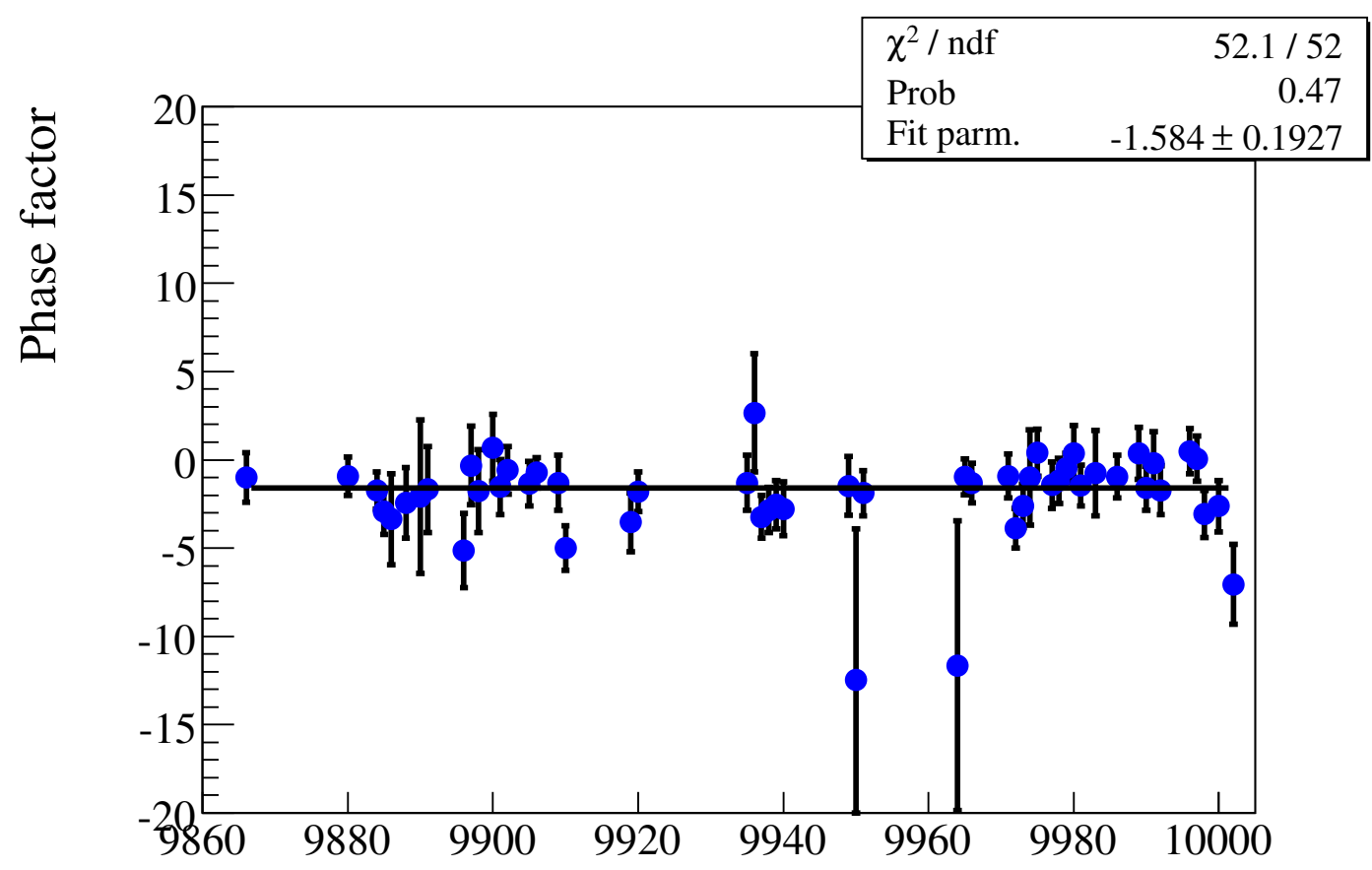

Fill number



Figure 12: Average $\phi_{0}$ of each fill for all scanned measurements (blue -top plot and yellow - bottom plot). 


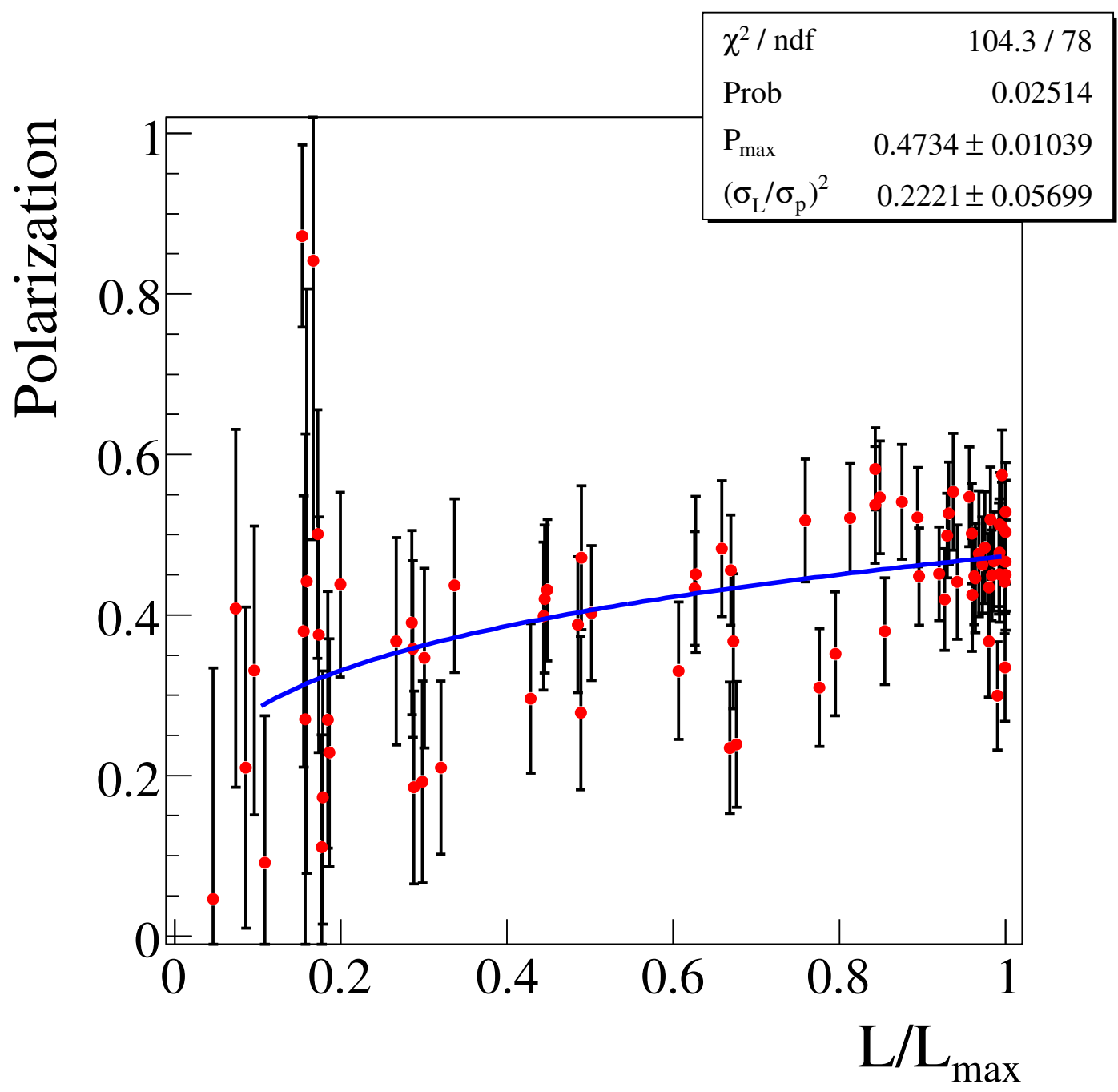

Figure 13: To extract $P_{\max }$ and $R$ a fit to $P$ Vs $\frac{I}{I_{\max }}$ was performed. The plot shows a typical fit to one fill. 


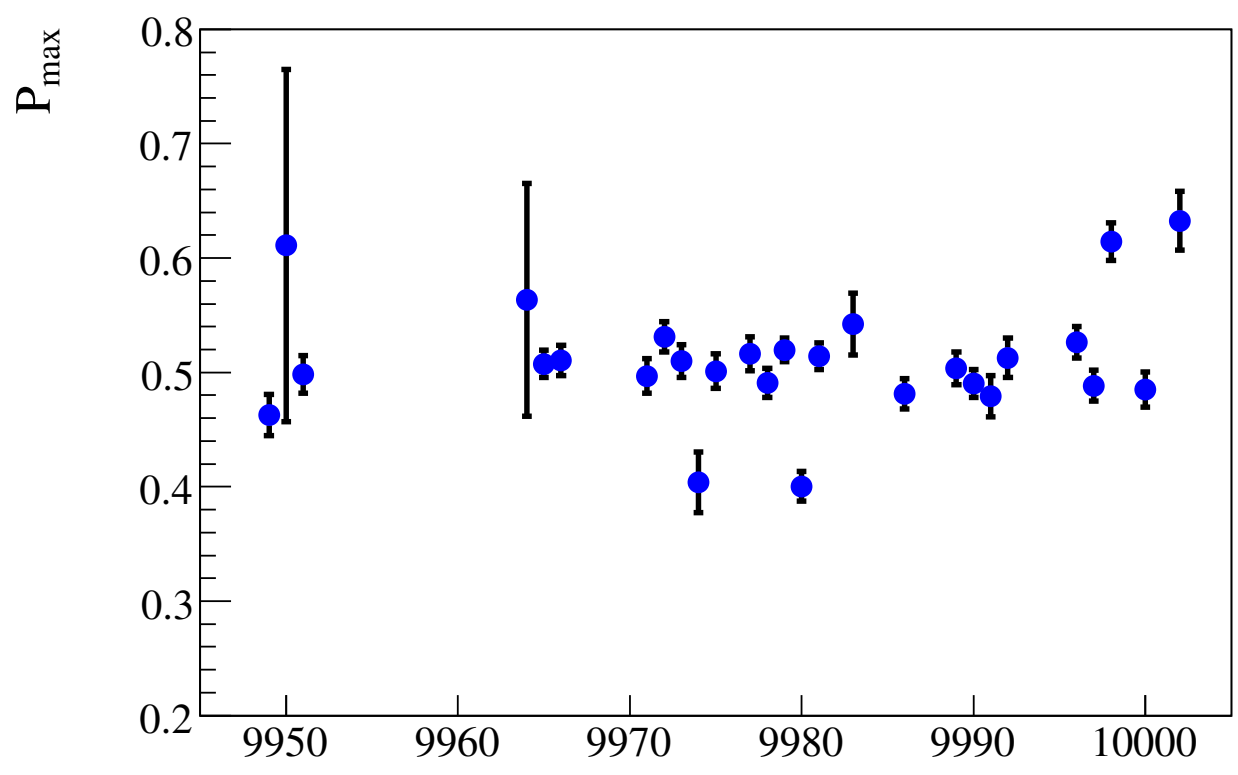

Fill number

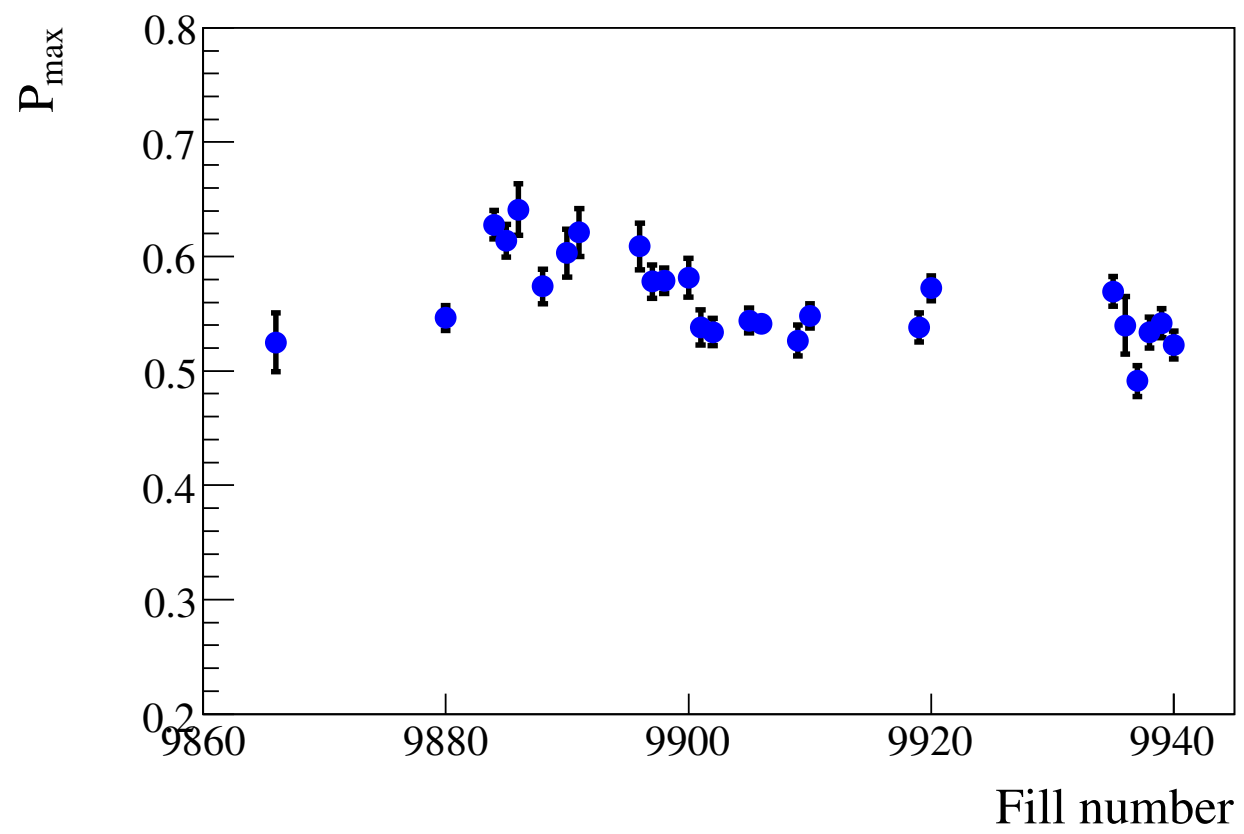

Figure 14: $P_{\max }$ history of (vertical scan (top) and horizontal scan(bottom)) all blue data. 


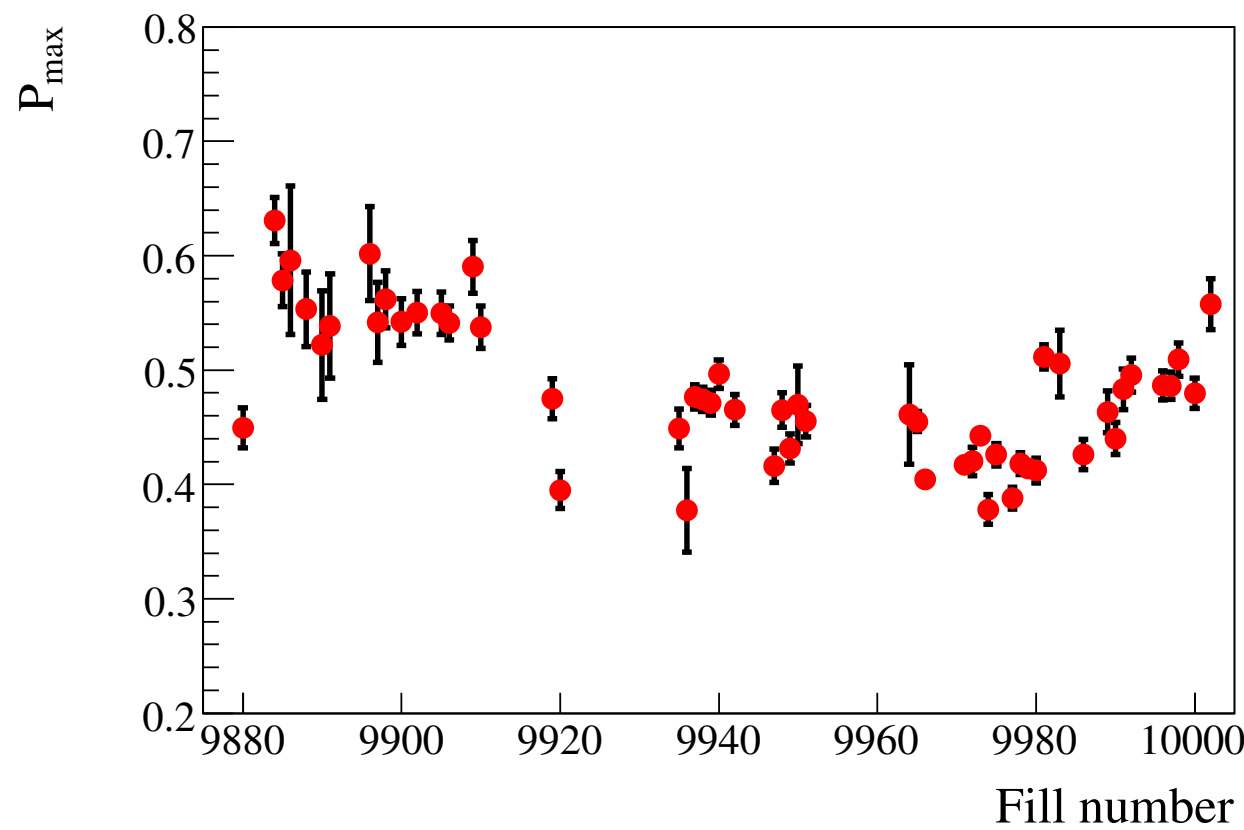

Figure 15: $P_{\max }$ history of (horizontal scan) all yellow data.

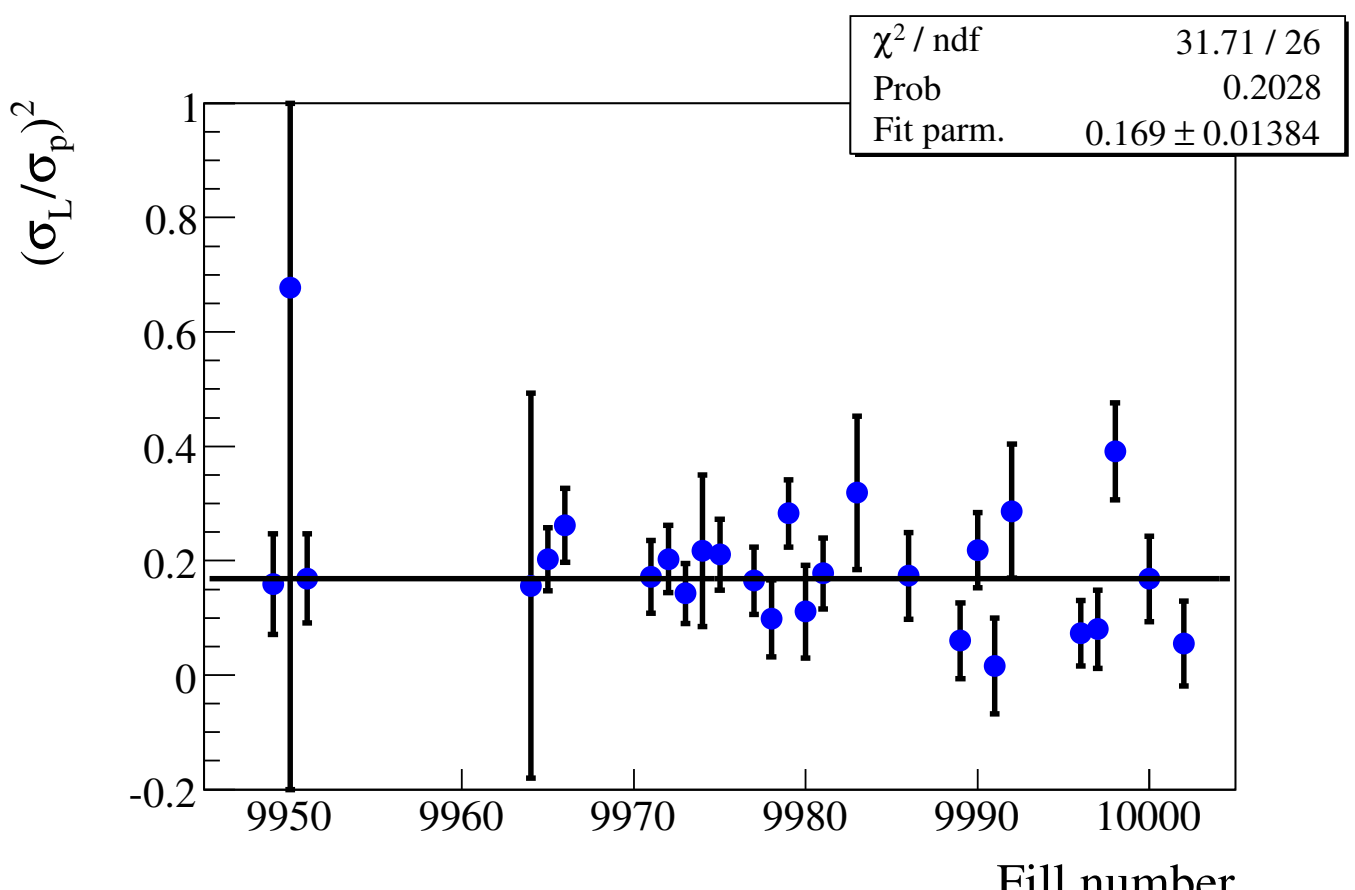

Figure 16: $R$ history of vertical scan blue data. 


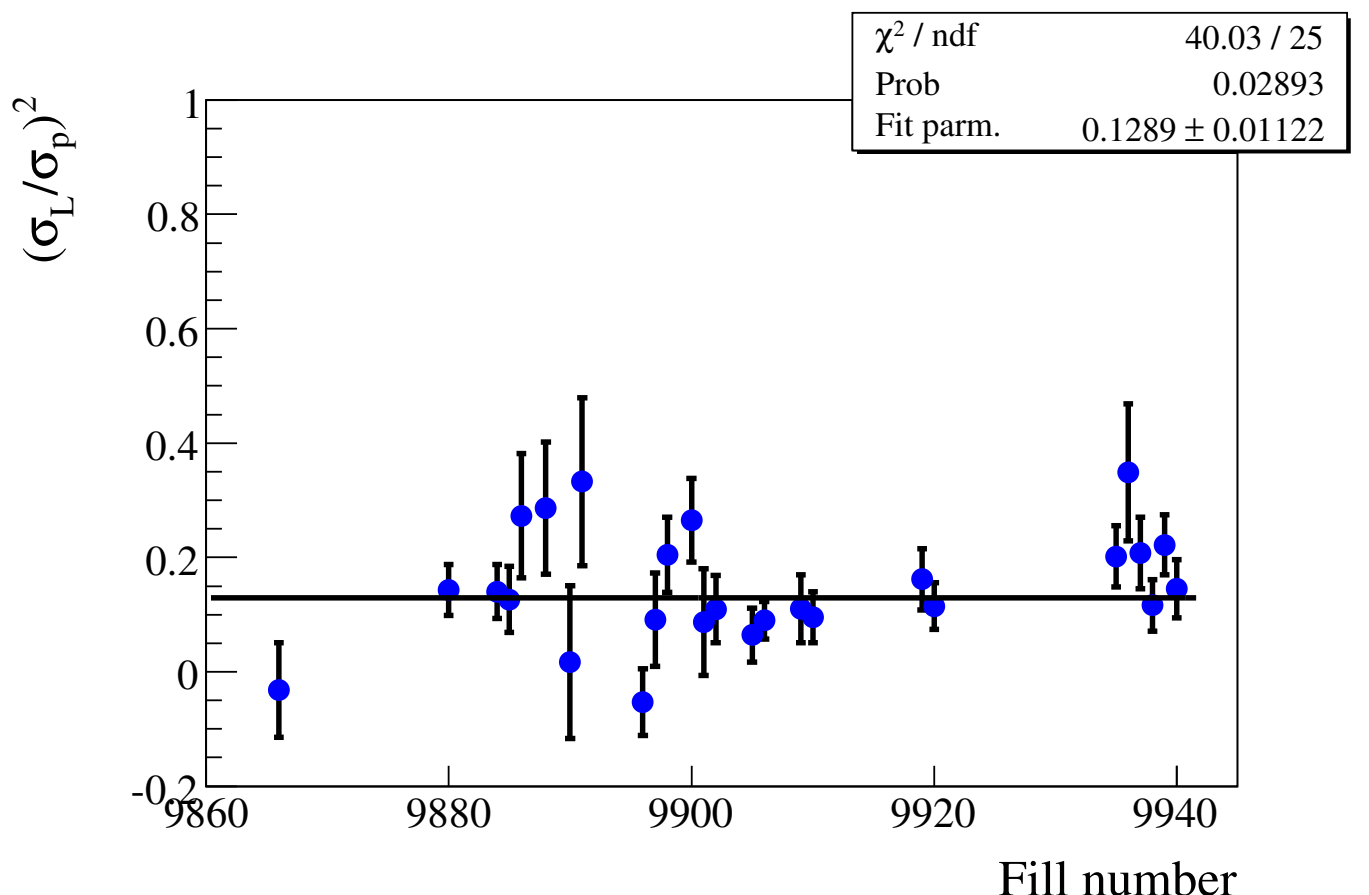

Figure 17: $R$ history of horizontal scan blue data.

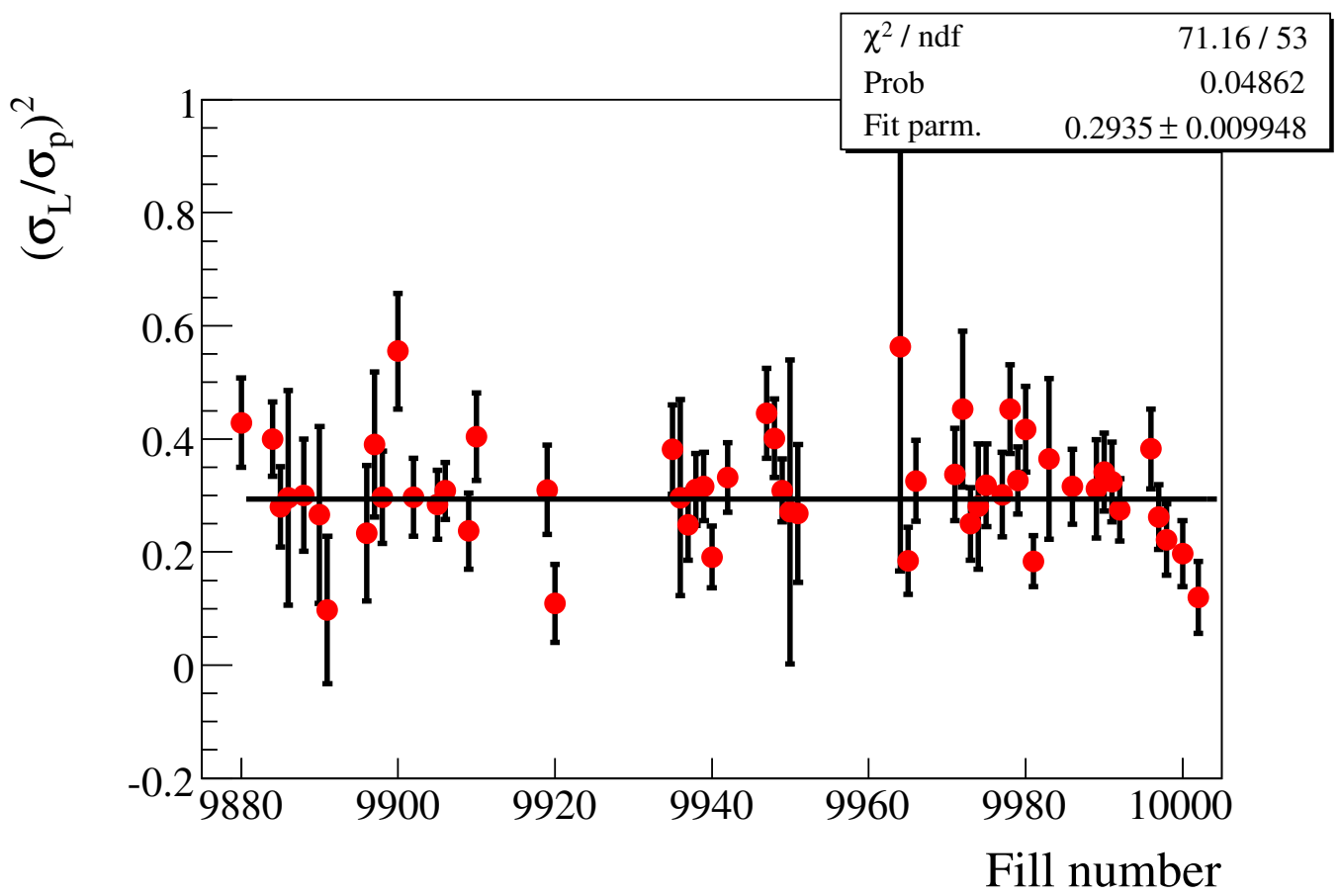

Figure 18: $R$ history of all (horizontal scan) yellow data. 

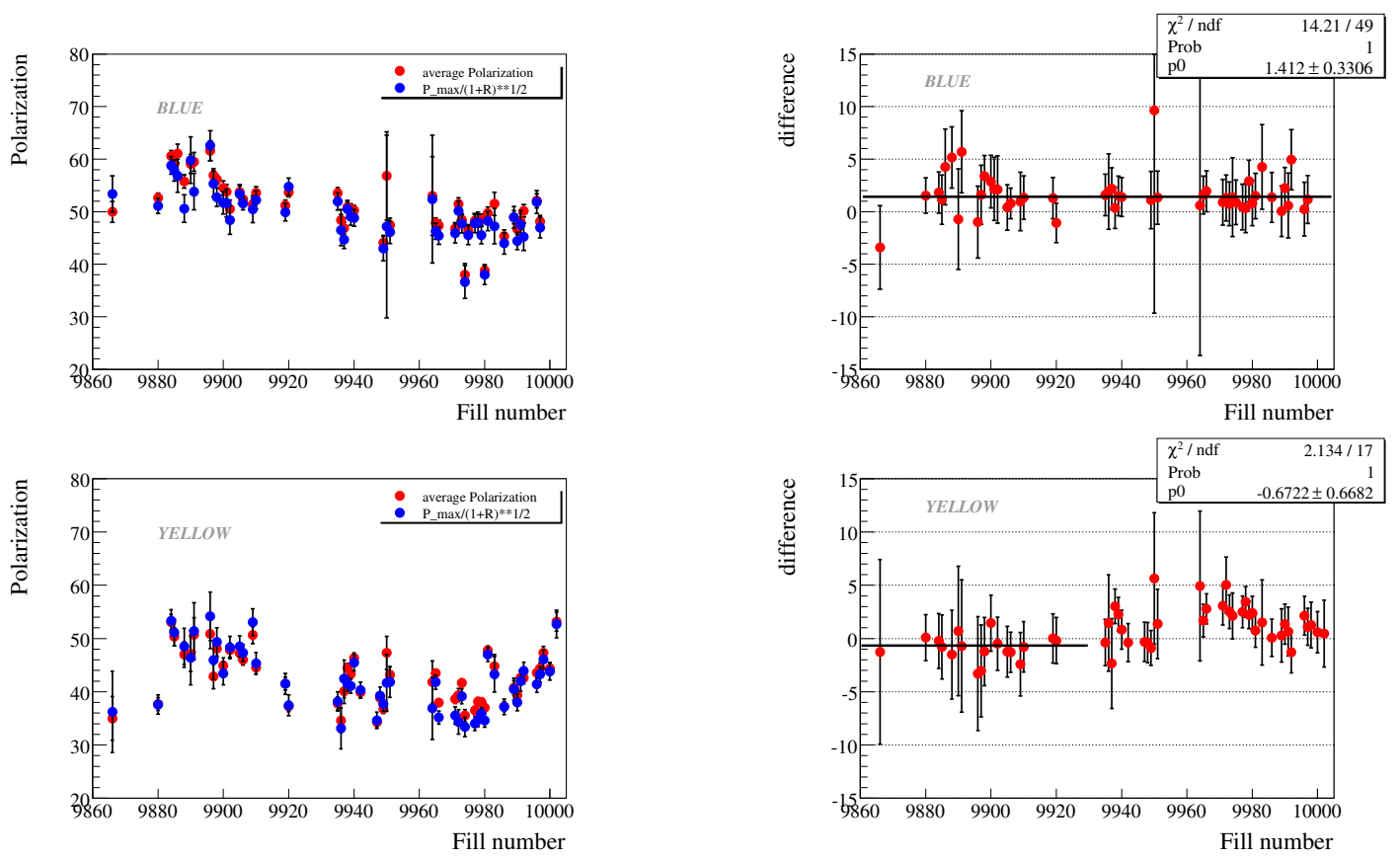

Figure 19: Average polarization calculated using fit parameters $P_{\max }$ and $R ;\langle P\rangle=$ $\frac{P_{\max }}{\sqrt{(1+R)}}$, and combining all scan measurements and the difference between the two methods for blue and yellow beams.

of the polarization was assumed. A fit of the form $P=P_{0} \exp ^{\alpha * t i m e}$, where $\alpha=1 / T_{\text {decay }}$ was performed to obtain the decay time $T_{\text {decay }}$ for each fill. The average polarization for fills that assume an exponential decay is given by: $\langle P\rangle=\frac{P_{0} \int e^{-\alpha P^{* t}} e^{-\alpha_{L} * t} d t}{e^{-\alpha_{L} * t} d t}$, where $\alpha_{L}$ and $\alpha_{P}$ are the inverse of the polarization and luminosity decay (a decay constant of 15 hours for luminosity decay was assumed to obtain the average polarization). Fig. 20 shows an example of a fill that shows large polarization decay and very little polarization decay. Fig. 21 shows the polarization decay for each fill. On average Yellow beam showed a much faster polarization decay than the blue beam. In addition, the average polarization obtained assuming no polarization decay and an exponential decay were compared. As it is evident from Fig. 21 the difference between the two are consistent with zero. It was concluded that any correction due to polarization decay is not necessary when averaging results over a given fill.

\section{Normalization to H-Jet}

To relate HJet measurements to $\mathrm{pC}$ measurements, only $R$ parameter in one direction (vertical or horizontal) is necessary, because the carbon target automatically averages polarization in the other direction. To determine the average pC polarization for each period, the pC polarization for each fill was weighted by number of HJet counts. Runs 

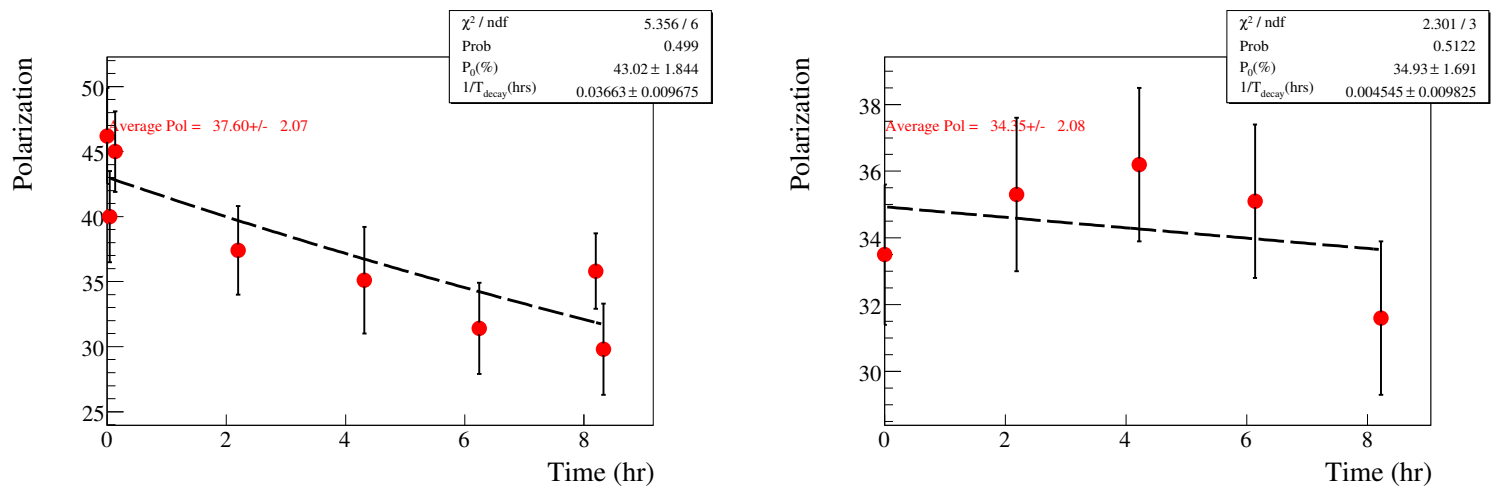

Figure 20: An example of a fill (Yellow fill 9880) that shows large polarization decay (left) and a fill (Yellow fill 9947) that shows relatively small polarization decay (right). Also shown are an exponential fit to data and the average polarization calculated assuming an exponential decay as described in the text.
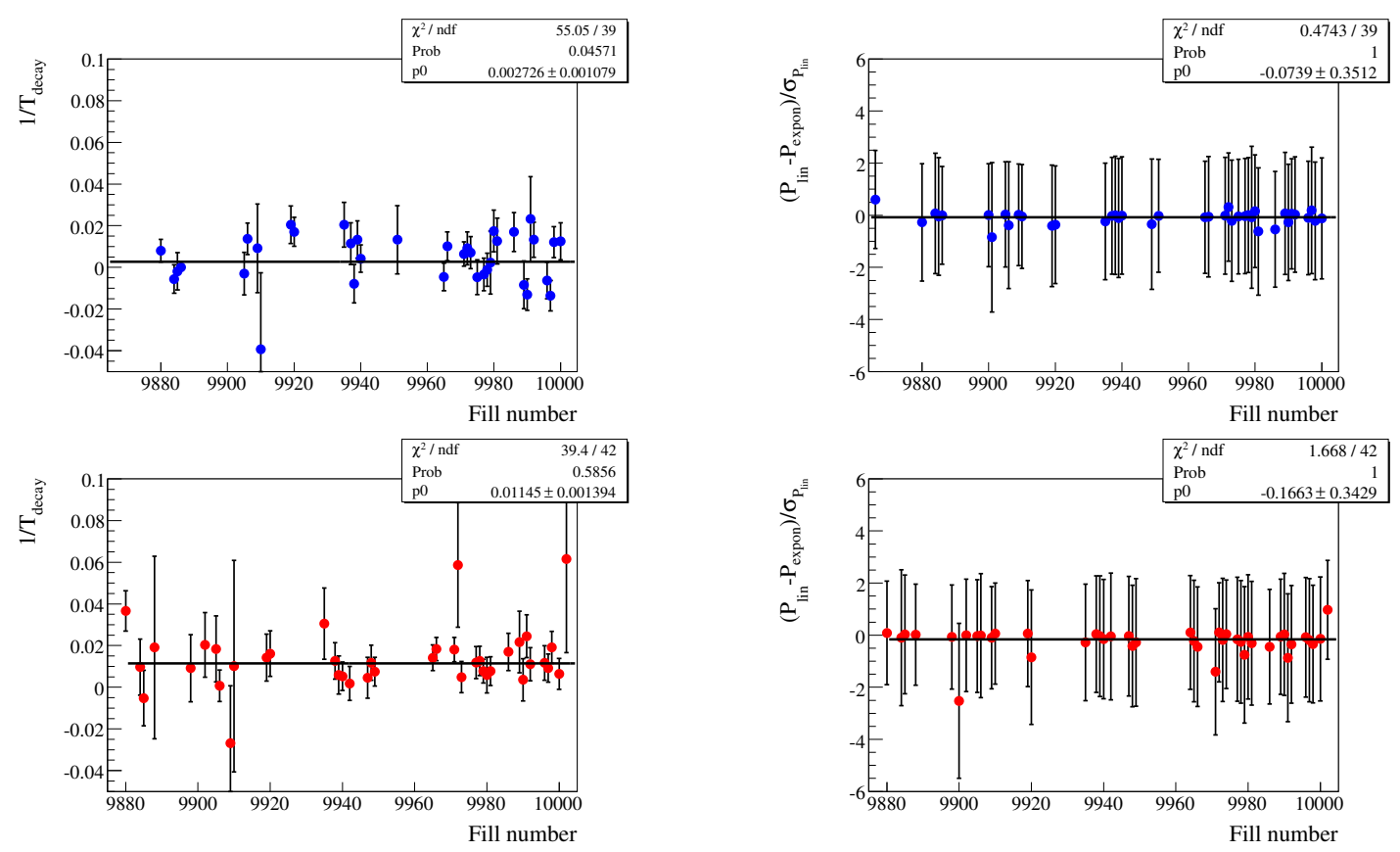

Figure 21: Polarization decay extracted for each fill assuming an exponential decay (top two plots). The difference of the average polarization calculated assuming no decay and an exponential decay is also shown (bottom two plots). See the text for more details. Red and blue markers correspond to yellow and blue beams respectively. 
with unfinished scans that did not reach the maximum intensity $\left(I_{\max }\right)$ as well as runs with unreliable measurements were excluded from calculation of $\mathrm{pC}$ to HJet normalization. A list of runs that were included in the analysis will be given elsewhere [2]. Four blue scan runs and eleven yellow scan runs that are "good" otherwise were not included in the analysis since the scan was unfinished and didn't reach the maximum of the Gaussian profile, as shown in Fig. 22. Tables 1 and 2 and Fig. 23 summarize the normalization constants for blue and yellow beams. Runs that did not have perfect scans and runs did have perfect scans were analyzed separately to determine the normalization constant. An examples of a scan that has perfect profile, scans that did not have perfect profiles but still were included in the analysis, and a run that was not included in the analysis due to incomplete profile are shown in fig. 22. The two normalization constants so obtained did not show any deviation within statistical errors. This confirmed that our technique to obtain average over transverse cross section beam polarization (via fit parameters $P_{\max }$ and $R$ ) is stable.


Figure 22: Examples of Luminosity profiles that has the expected Gaussian shape (top left), unfinished scan that was not included in the analysis (top right), and a flat profile and an unfinished scan that were considered acceptable and therefore were included in the analysis (bottom). 
Table 1: Normalization constant pC/HJet (blue) for different periods

\begin{tabular}{|c|c|c|c|c|}
\hline \hline Period & pC average & HJet & $\begin{array}{c}\text { normalization } \\
\text { constant } \\
\text { pC/HJet }\end{array}$ & Fills \\
\hline 1 & $52.02 \pm 0.64$ & $47.93 \pm 2.37$ & $1.086 \pm 0.055$ & $\begin{array}{c}9885,9888,9890, \\
9898,9902,9905, \\
9906,9909\end{array}$ \\
\hline 2 & & & $\begin{array}{c}\text { Did not calculate } \\
\text { (one fill) }\end{array}$ & $\begin{array}{c}9949 \\
\text { (9942 and } 9948 \\
\text { are not included) }\end{array}$ \\
\hline 3 & $47.02 \pm 0.74$ & $43.15 \pm 1.62$ & $1.090 \pm 0.044$ & $\begin{array}{c}9972,9973,9974,9975, \\
9977,9978,9979\end{array}$ \\
\hline 4 & $47.80 \pm 1.38$ & $48.70 \pm 2.43$ & $0.982 \pm 0.057$ & $9991,9992,9996$ \\
\hline ALL & $47.96 \pm 0.53$ & $45.17 \pm 1.11$ & $1.062 \pm 0.029$ & periods 1+2+3+4 \\
\hline \hline
\end{tabular}
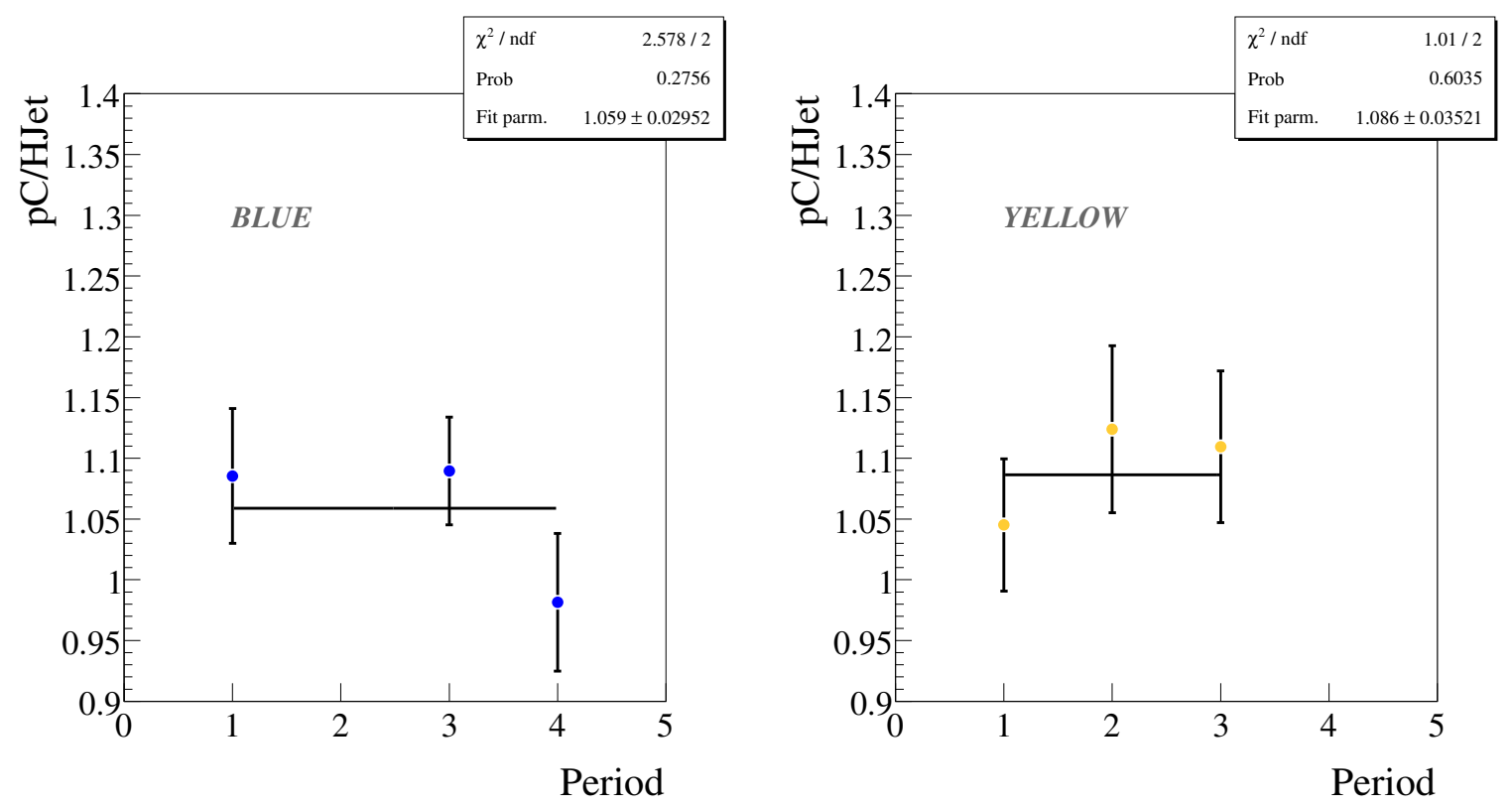

Figure 23: Comparison of normalization constant $\mathrm{pC} /$ HJet for different periods as defined in Tables 1 and 2 .

\section{Polarization for experiments}

After normalization for $\mathrm{pC}$ measurements is obtained, the last step is to provide polarization values for experiments which are averages obtained weighting with a prod- 
Table 2: Normalization constant pC/HJet (yellow) for different periods

\begin{tabular}{|c|c|c|c|c|}
\hline \hline Period & pC average & HJet & $\begin{array}{c}\text { normalization } \\
\text { constant } \\
\text { pC/HJet }\end{array}$ & Fills \\
\hline 1 & $41.00 \pm 0.61$ & $39.23 \pm 1.96$ & $1.045 \pm 0.054$ & $\begin{array}{c}9919,9920,9935, \\
9937,9938,9939, \\
9940\end{array}$ \\
\hline 2 & $38.16 \pm 0.73$ & $33.95 \pm 1.97$ & $1.124 \pm 0.069$ & $\begin{array}{c}9951,9965,9966, \\
9971 \\
3\end{array}$ \\
& $39.55 \pm 0.69$ & $35.65 \pm 1.90$ & $1.110 \pm 0.062$ & $\begin{array}{c}9980,9981,9986, \\
9989,9990\end{array}$ \\
\hline ALL & $39.59 \pm 0.39$ & $36.30 \pm 1.12$ & $1.091 \pm 0.035$ & periods $1+2+3$ \\
\hline \hline
\end{tabular}

uct of two beam intensities in both $\mathrm{x}$ and $\mathrm{y}$ transverse dimensions. For the simple case when the transverse size $\left(\sigma_{I}\right)$ is about the same in yellow and blue beams: $<P>=\frac{P_{\max _{2}}}{\sqrt{\left(1+R_{x} / 2\right) \sqrt{\left(1+R_{y} / 2\right)}}}$, where $R_{x}$ and $R_{y}$ are $\left(\frac{\sigma_{I}}{\sigma_{P}}\right)^{2}$ in horizontal and vertical direction respectively, and $P_{\max _{2}}$ - is polarization at the intensity peak in two dimensional

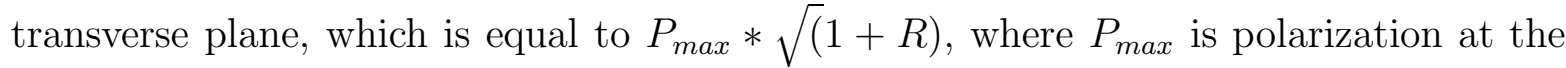
intensity peak in one dimensional case (integrated over the perpendicular direction). Therefor to extract polarization for experiments, the knowledge on polarization profile in both transverse directions is necessary. Since we only have $R$ in either vertical or horizontal direction for each fill, the unmeasured direction was estimated using known values from other fills.

As it is shown on Figs. [16, 17, 25], polarization profile parameter $R$ is about the same for both vertical and horizontal directions. It was decided to use $R=0.15$ for all unmeasured profiles in blue. Possible fill-by-fill variation in $R$ from Fig. 25 of \pm 0.11 (to accommodate $\pm R M S$ in both vertical and horizontal profiles) was used to assign the fill-by-fill uncertainty to polarization measurements, due to unmeasured profile. Here we should mention that the large contributor to RMS of $R$ distributions is statistical fluctuations. Therefore attributing the RMS value to fill by fill variations, will be an overestimation of these variations. The \pm 0.02 uncertainty in the average unmeasured polarization profile (since average $R$ was obtained based on approximately half of the fills and applied to the other half, where it was not measured) is a source of global uncertainty in polarization measurements to be discussed below.

Unfortunately, for the yellow beam only horizontal scans are available. As it is seen in Fig. 26 the $R_{\text {hor }} \pm R M S \sim 0.30 \pm 0.10$. Since we do not have data to determine how sharp the vertical polarization profile for yellow is, we assumed $R_{\text {vert }}$ could possibly vary from 0 to $R_{\text {hor }}+R M S$, which is 0 to 0.40 . Therefore it was decided to use middle 
point $R_{\text {vert }}=0.20$ with possible fill-by-fill variation of \pm 0.20 (which goes to fill-by-fill uncertainty), and with the same uncertainty \pm 0.20 for the average $R_{\text {vert }}$ (which goes to global polarization uncertainty).

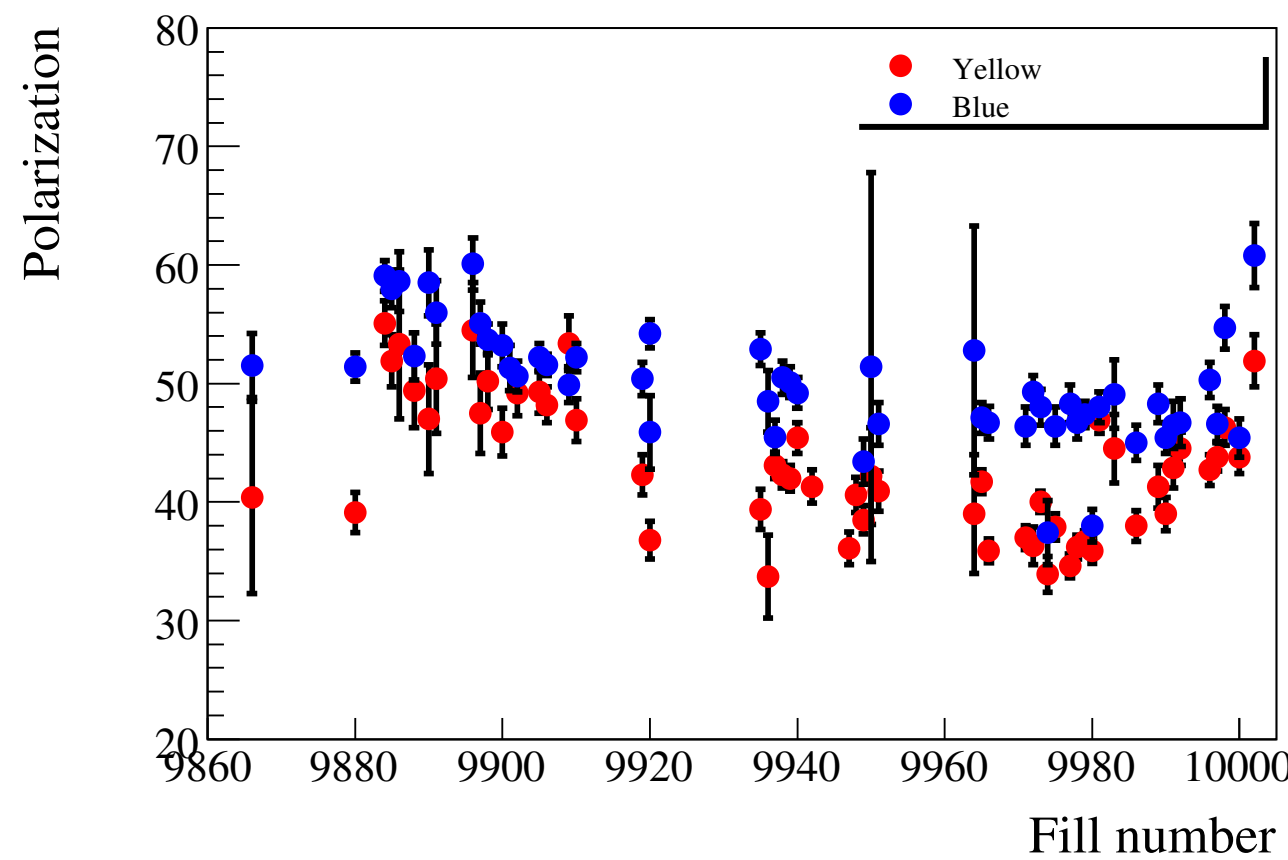

Figure 24: Polarization for experiments calculated as described in the text. Errors only indicate statistical uncertainties.

\section{Systematic uncertainties}

Main systematic uncertainties in pC polarization measurements are due to the uncertainties in polarization profile and the energy correction (dead layer). Some of the uncertainties due to polarization profile are similar to that of run 6 and a similar approach for run 8 was adopted (total 1.1\%):

- In non-continuous scans we may miss the maximum, which might lead to wrong $I_{\text {max }}$; Uncertainty due to this is $\approx 0.2 \%$

- For several runs a flat luminosity profile, instead of a Gaussian peak was observed. One of the possible reasons could be target vibrations, giving average over a certain distance rather than a measurement at one fixed place across the beam. A simulation was performed to determine uncertainties due to this [3]. And the upper limit for this contribution to polarization measurements was determined to be $\approx 1 \%$. 
- An uncertainty of $0.5 \%$ was assigned to take into account the assumption that the profiles are Gaussian.

The main uncertainty due to polarization profile arises due to variation in $R$ and also due to having the profile information for either horizontal or vertical profile. As we discussed in previous section the $\pm 0.11( \pm 0.20)$ fill by fill possible fluctuations in unmeasured polarization profile in blue (yellow) beams lead to $\sim 3 \%(\sim 5 \%)$ uncertainty in polarization measurements for experiments, in which the parameter $R$ enters as $1 / \sqrt{1+R / 2}$. The uncertainties for average $\mathrm{R}$ (over all measurements) for blue (yellow) beams are $\pm 0.02( \pm 0.20)$. In addition, $R$ for unmeasured profile lead to a global uncertainty of $\sim 0.5 \%(\sim 5 \%)$ in polarizations for experiments.
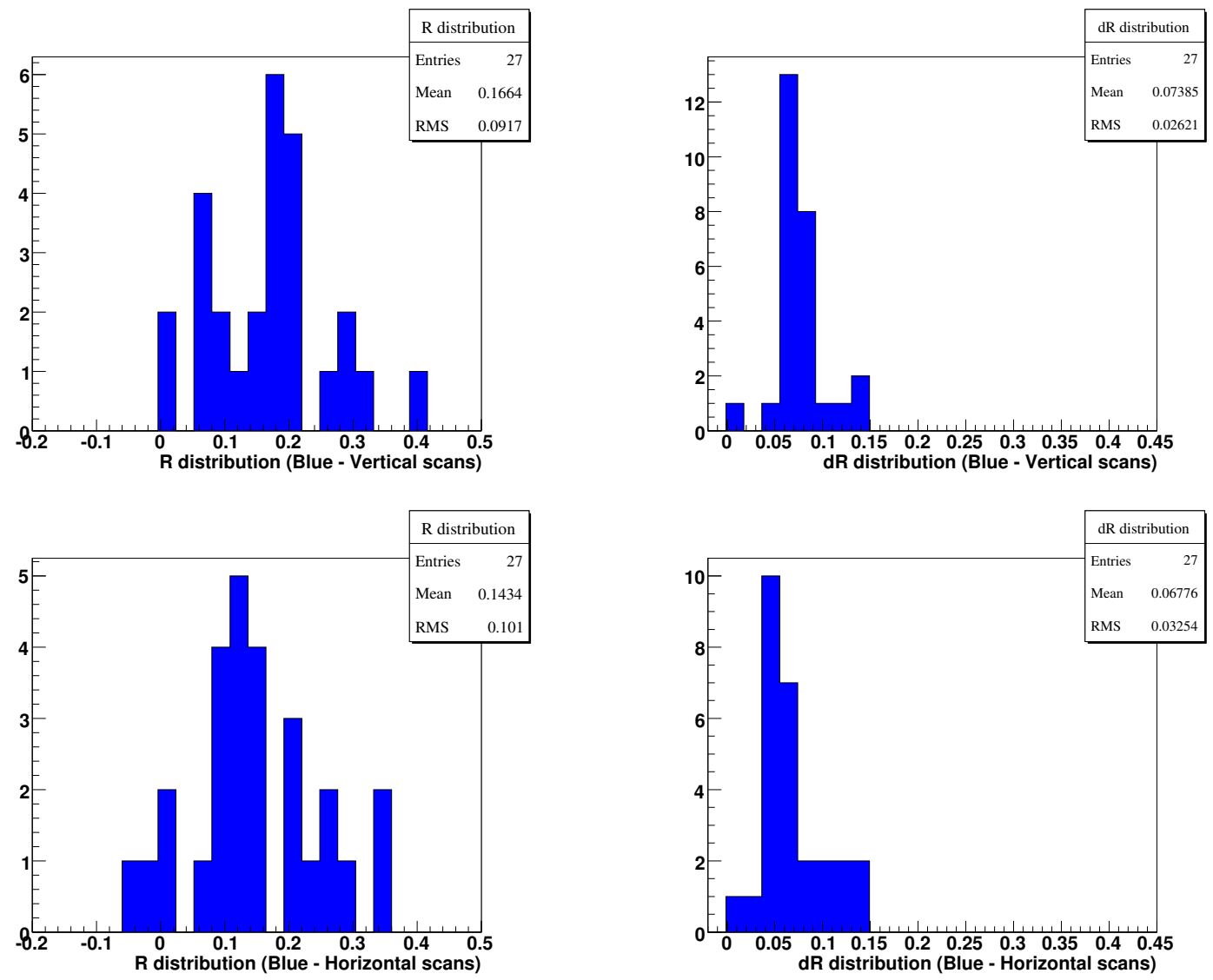

Figure 25: Distribution of $R$ and the errors of $R$ for blue vertical (top plots) and blue horizontal (bottom) scans.

The other systematic uncertainty as described above is due to the fill to fill variation of the energy correction (dead layer). Fig. 27 shows the dead layer history for all scan measurements that were included in the analysis. On average the energy correction for the blue (yellow) detectors varied $\pm 3 \mu \mathrm{g} / \mathrm{cm}^{2}\left( \pm 4 \mu \mathrm{g} / \mathrm{cm}^{2}\right)$ from the average, that corresponds to $1.8 \%(2.4 \%)$ uncertainty. This is based on an average $0.6 \%$ effect on 

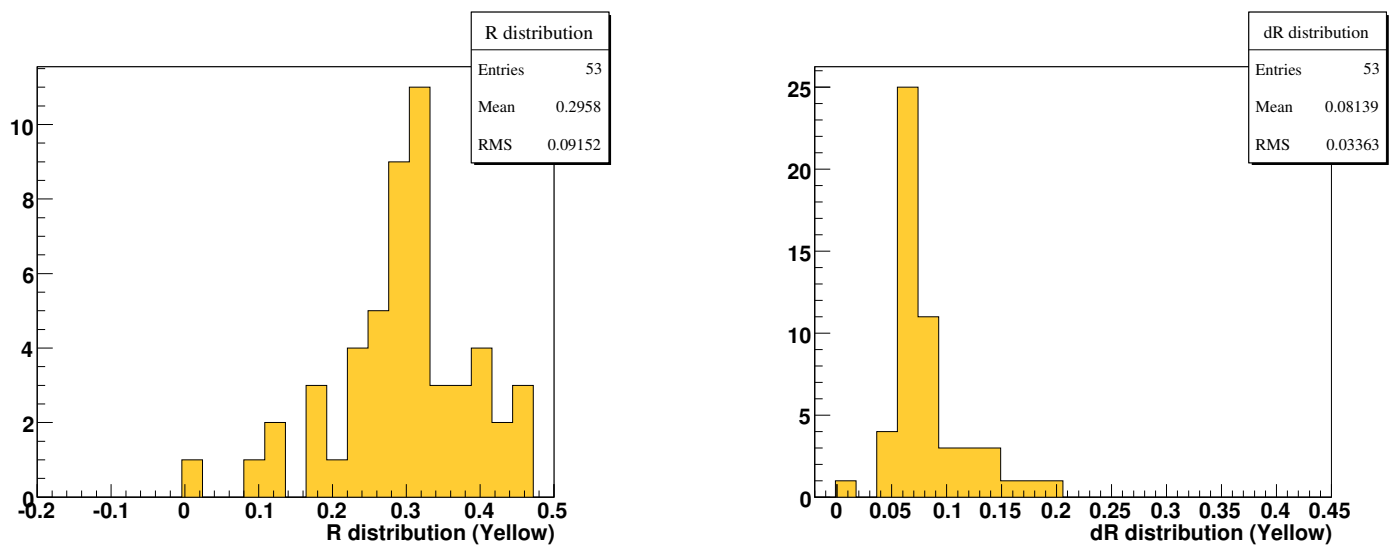

Figure 26: Distribution of $R$ and the errors of $R$ for yellow horizontal scans.

polarization from $1 \mu \mathrm{g} / \mathrm{cm}^{2}$ change in DL. Assigning these global uncertainties assumes our poor knowledge on why the dead layer drifts over time, therefore a $100 \%$ uncertainty was given for those corrections. Deviation of the dead layer correction from a linear fit to dead layer Vs fill number was used to determine the fill to fill uncertainties. Fig. 28 shows the distribution of the deviation. The RMS values of the distributions $\left(1.6 \mu \mathrm{g} / \mathrm{cm}^{2}\right.$ and $1.1 \mu \mathrm{g} / \mathrm{cm}^{2}$ ) were used to assign errors, which are $1 \%$ and $0.7 \%$ for blue and yellow respectively. Table 3 and 4 summarize the final systematic uncertainties.
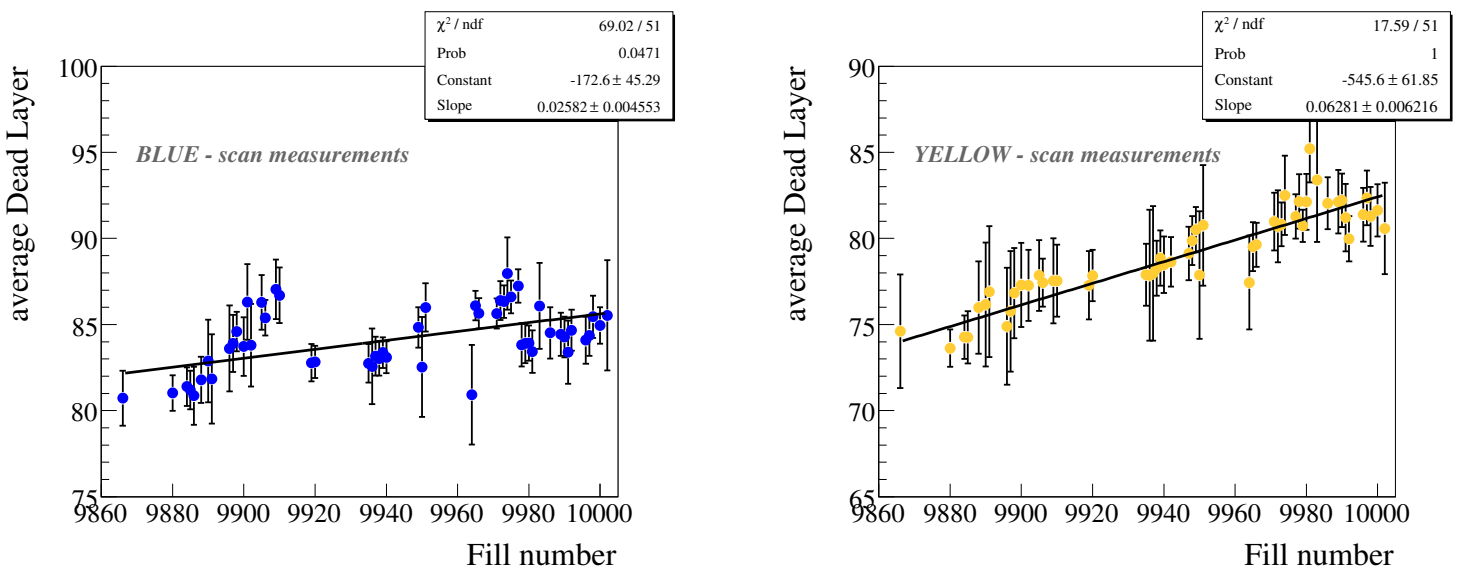

Figure 27: Dead layer (averaged over fills) history of all the scan measurements that were included in the analysis.

The final global uncertainties, deltaP/P, are:

Blue: $4.2 \%$

Yellow: $7.2 \%$

Considering that "Jet normalization, systematic" as well as "Energy correction" uncertainties are mostly correlated between blue and yellow, the final global uncertainties for 

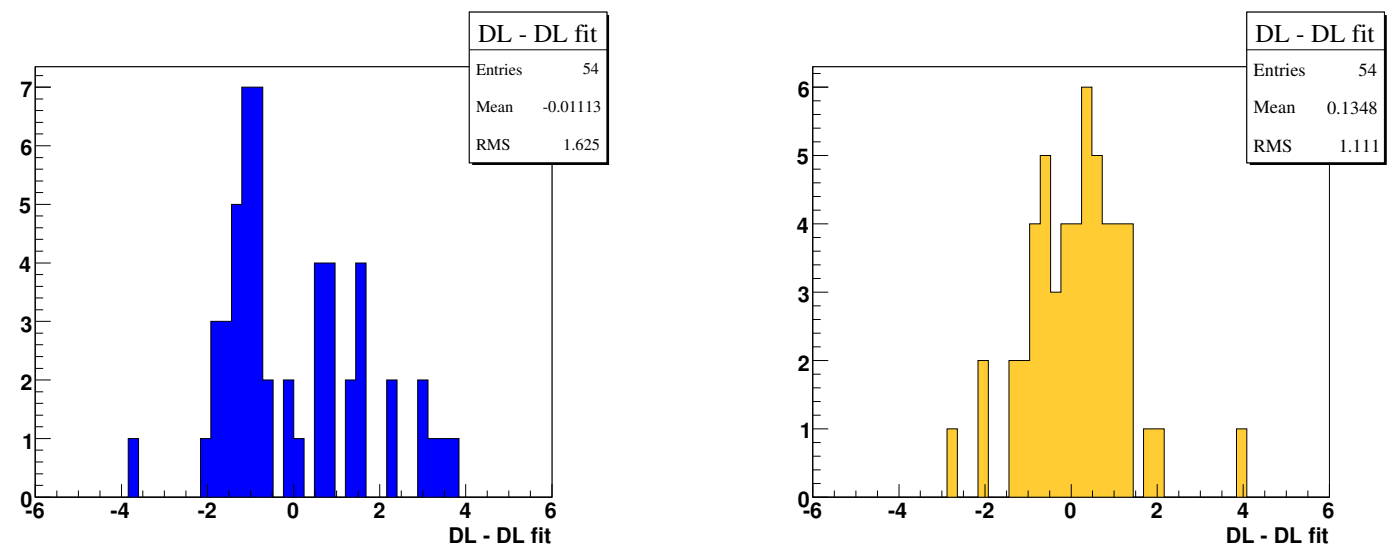

Figure 28: Distribution of the deviation of dead layer from a fit (as shown in Fig. 27 to dead layer history.

Table 3: fill-by-fill (non-correlated) systematic uncertainties

\begin{tabular}{ccc}
\hline \hline & & \\
& Blue & Yellow \\
\hline From vert/horizontal profile & $3 \%$ & $5 \%$ \\
Energy correction & $1 \%$ & $0.7 \%$ \\
\hline \hline
\end{tabular}

a product of two beams, $\delta\left(P_{B} * P_{Y}\right) /\left(P_{B} * P_{Y}\right): 9.6 \%$

\section{References}

[1] I. Nakagawa et al., RHIC/CAD Accelerator Physics Note Vol.275 (2007)

[2] pC Run-8 run summary

[3] A. Bazilevsky, analysis of Run $6 \mathrm{pC}$ systematic errors http://www4.rcf.bnl.gov/ cnipol/pubdocs/Run060ffline/RSC_30nov2007_sasha.ppt RHIC/CAD Accelerator Physics Note 298 
Table 4: Global systematic uncertainties (correlated from fill to fill)

\begin{tabular}{ccc}
\hline \hline & & \\
& Blue & Yellow \\
\hline Jet normalization, stat & $2.7 \%$ & $3.2 \%$ \\
Jet normalization (profile) & $1.1 \%$ & $1.1 \%$ \\
Jet normalization, syst (molecular) & $2.0 \%$ & $2.0 \%$ \\
Jet normalization, syst (other) & $1.3 \%$ & $2.4 \%$ \\
Pol. profile (vert. for exp) & $0.5 \%$ & $5.0 \%$ \\
Energy correction: & $1.8 \%$ & $2.4 \%$ \\
\hline \hline
\end{tabular}

